\title{
Long Period Trajectories in the Seasonal SIR Model
}

\author{
by \\ Lucas Finney
}

A thesis submitted to the Faculty of Graduate and Postdoctoral Affairs in partial fulfillment of the requirements for the degree of

\section{Master of Science}

in

Mathematics

Carleton University

Ottawa, Ontario

(C) 2021

Lucas Finney 
The Plague: Men love a world of men. But life is more than men. Life is manifold, overflowing. Flowing from body to body. You call this work "illness."

Pathologic 2, by Nikolay Dybowski 
Many infectious diseases exhibit seasonally varying incidence rates. In order to mathematically describe and predict the behaviour of such diseases, the seasonal susceptible-infected-removed (Seasonal SIR) model, a system of ordinary differential equations, was formulated. It has been found in numerical simulations of the Seasonal SIR model that sub-harmonic bifurcations may occur, leading to the appearance of periodic modes with inter-outbreak periods on the scale of two time units or more in response to a varying forcing of period one.

In the extremes of these long inter-outbreak period modes, it has been observed that periodic modes on the order of nine time units or more may be found. Additionally, these long interoutbreak period modes exhibit a behaviour of increasing outbreak severity as the time between outbreaks is lengthened.

In order to investigate the qualitative behaviour of such modes as the system parameters are varied, we have divided the interval from outbreak-to-outbreak into an outbreak region and an inter-outbreak region. We have shown that existing analytic approximate solutions are sufficient for capturing the behaviour of the model during the outbreak region. Additionally, we have shown that the application of a dominant-balance argument and appropriate scaling may transform the system into a solvable form in the inter-outbreak period, using approximations that hold in the long inter-outbreak limit investigated.

We have shown that it is possible to construct a Poincaré mapping for use in determining the existence of stable periodic modes, and that it is possible to predict the spectrum of available stable modes. Furthermore, we have determined that for a given set of parameters, a finite spectrum of stable modes is available, with the inter-outbreak period increasing as strength of the seasonal forcing is varied. 


\section{Acknowledgements}

I would like to thank my supervisor, David Amundsen, for his consistent support and inspiration, wherever my hunt took me. Further, I would like to thank Carleton University for the incredible opportunity which has been afforded to me.

I want to thank Ice-Pick Lodge, the developers of the video game "Pathologic 2", for providing the seed of inspiration for this paper. Because of them, I can claim the time I spent in that world was research, rather than procrastination.

Additionally, I must thank my family — Kenneth, Jacqueline, and Indiana Finney — and my friends, particularly Cameron, Jess, and Nic (roll a sanity check against the horrifying math you may glimpse herein!) for their support and understanding throughout this journey.

Lastly, I want to thank my partner, Ginger, for her endless love and for pointing out the collection of grey hairs I've grown throughout the process. 


\section{Table of Contents}

$\begin{array}{lr}\text { List of Figures } & \text { vi }\end{array}$

$\begin{array}{lc}\text { List of Tables } & \text { vii }\end{array}$

1 Introduction $\quad 1$

2 Mathematical Background $\quad 4$

2.1 Lambert $W$ Function . . . . . . . . . . . . . . . . . . . . . . . 4

2.2 Asymptotic Relations . . . . . . . . . . . . . . . . . . . . . . 4

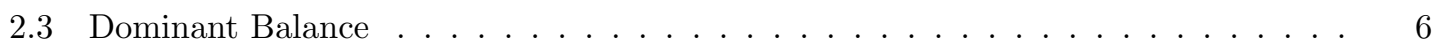

3 Application Background $\quad 7$

3.1 The SIR Model . . . . . . . . . . . . . . . . . . . . . 7

3.2 The Vital SIR (V-SIR) Model . . . . . . . . . . . . . . . . . . . . . . 9

3.3 The Seasonal SIR (S-SIR) Model . . . . . . . . . . . . . . . . . . . . . . . 10

4 Numerical Simulations and the Guiding Question $r$

5 Construction of Approximate Solutions $\quad 16$

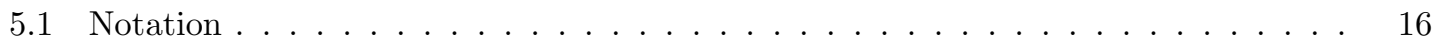

5.2 Inter-Outbreak Region $(\mathrm{II}) \quad \ldots \ldots \ldots \ldots \ldots \ldots$

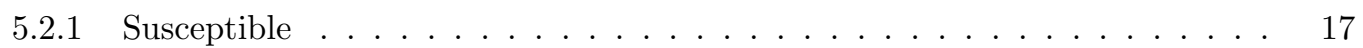

5.2 .2 Infected . . . . . . . . . . . . . . . . . 17

5.2 .3 Dominant Balance Confirmation . . . . . . . . . . . . . . . . . 19

5.3 Outbreak Region . . . . . . . . . . . . . . . . . . . . 23

5.3.1 Simplifying Assumptions . . . . . . . . . . . . . . . . . 23

5.3 .2 Solving the SIR Model . . . . . . . . . . . . . . . . 25

5.3.3 Verifying the Formal Solution . . . . . . . . . . . . . . . . . 26 
6.1 Finding the Peak Infection Rate, $\psi_{0} \ldots \ldots \ldots \ldots \ldots$

6.2 Approximating $t(G) \ldots \ldots \ldots \ldots \ldots$

6.3 Finding the Time of Peak Infection, $t_{p} \ldots \ldots \ldots . \ldots \ldots$

6.4 Approximating $G(t) \ldots \ldots \ldots \ldots \ldots \ldots$

$6.4 .1 \quad$ Finding $g_{1}(t) \ldots \ldots \ldots \ldots \ldots \ldots$

6.4 .2 Finding $g_{2}(t) \ldots \ldots \ldots \ldots \ldots$

6.4 .3 Statement of $\tilde{G}(t) \ldots \ldots \ldots \ldots \ldots$

6.5 The Street-Fighting Outbreak Solution, $\tilde{E}_{I}(t) \ldots \ldots \ldots$. . . . . . . . . . 40

6.6 Finding a Uniformly Valid $\tilde{S} \ldots \ldots \ldots$. . . . . . . . . . . . . . . 41

7 Evolution of Long-Period Population Orbits $\quad 44$

7.1 Determining Post-Outbreak Populations . . . . . . . . . . . . . . . . . 44

7.1.1 Modifying the Outbreak Approximation . . . . . . . . . . . . . 44

7.1.2 Calculating the Initial Infected Population . . . . . . . . . . . . . . . 45

7.1.3 Determining the Outbreak Endpoint: Method A . . . . . . . . . . 45

7.1.4 Determining the Outbreak Endpoint: Method B . . . . . . . . . . 46

7.2 Finding the Inter-Outbreak Region Using the Outbreak Width . . . . . . . . . . 47

7.3 Poincaré Mapping and Variational Form . . . . . . . . . . . . . . . . . . . 47

8 Results $\quad 48$

9 Discussion $\quad 53$

9.1 Comparison to Literature . . . . . . . . . . . . . . . . . . . 53

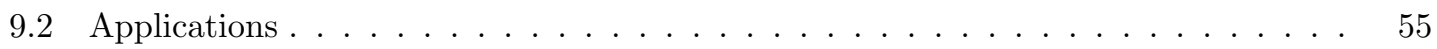

9.3 Future Research Opportunities . . . . . . . . . . . . . . . . . . 57

10 Conclusion 


\section{List of Figures}

1 The principal branch of the Lambert $\mathrm{W}$ function . . . . . . . . . . . . . 5

2 Initial development of the SIR model for $\left(\beta_{0}, \gamma\right)=(500,40)$ and $(S(0), I(0))=$

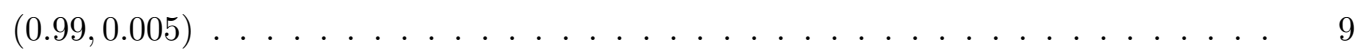

$3 \quad$ V-SIR solution development over varying time scales . . . . . . . . . . . . . 10

4 S-SIR solution development over varying time scales. . . . . . . . . . . . . . . 11

5 Three different possible trajectories for $S(t)$ over time . . . . . . . . . . . 13

6 Period 16 Mode . . . . . . . . . . . . . . . . . . . 14

7 Close-Up on Outbreak region in Figure 6. $\left(\beta_{0}, \delta, \gamma, \mu\right)=(500,0.9,40,0.01)$. . . . 14

8 Logarithmically scaled $I(t)$ for the period 16 mode, $\left(\beta_{0}, \delta, \gamma, \mu\right)=(500,0.9,40,0.01) \quad 15$

$9 \quad$ Comparing $\ln I(t)$ obtained by numerical solution vs. using the approximate solution. 19

$10 \ln \tilde{I}_{\mathrm{II}}(t)$ plotted for varying values of $\gamma \ldots \ldots \ldots \ldots$

11 ln-scaled ratio of $\tilde{S}(t)$ and $\tilde{I}(t),\left(\beta_{0}, \delta, \gamma, \mu\right)=(500,0.9,40,0.01) \ldots \ldots 21$

12 Comparing $\ln I(t)$ obtained by numerical solution vs. using the full approximate solution vs. using the simplified approximation from equation (28) . . . . . . . . 22

13 Comparing the solution to the full seasonal SIR model and the traditional SIR model 24

14 Plotting discrete points of $\mathrm{S}(\mathrm{t})$ and $\mathrm{I}(\mathrm{t})$ from the formal solution (36) and numerical solution, $\left(\beta_{0}, \delta, \gamma, \mu\right)=(500,0.9,40,0.01) \ldots \ldots \ldots \ldots$

15 Comparing $G_{0}$ and the approximate $\tilde{G}_{0} \ldots \ldots \ldots \ldots$

16 Comparing $\gamma / f(x)$ (solid) and $1 / h_{1}(x)+1 / h_{2}(x)$ (dashed) for varying $R_{0} \ldots \ldots 32$

17 Comparing $\gamma / f(x)$ (solid) $\gamma / h_{1}(x)$, and $\gamma / h_{2}(x)$ (dashed) for varying $R_{0}$, plotted over the entire domain . . . . . . . . . . . . . . . . . . 33

18 Comparison of $\gamma \int_{0.01}^{G_{\infty}} f(x)^{-1} d x$ (solid) and $\gamma \int_{0.01}^{G_{\infty}} h_{1}(x)^{-1}+h_{2}(x)^{-1} d x$ (dashed) for varying $R_{0} \ldots \ldots \ldots \ldots \ldots \ldots \ldots \ldots$ 
19 Plot of the difference between $\ln (1-g)$ and $\frac{\ln g\left(1-g_{0}\right)}{g_{0}} \ldots \ldots \ldots . \ldots . \ldots$

20 Plotting the difference between $\ln g$ and the approximation in equation (88). . . . 39

21 Comparing the numerical solution to $\tilde{E}_{I}(t)$ in the outbreak region $\left(\beta_{0}, \delta, \gamma, \mu\right)=$

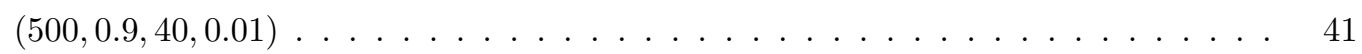

22 Numerical $S(t)$ and $\tilde{S}_{U}(t)$ as in equation (101) plotted over more than one period . 43

$23 S_{0}-P\left(S_{0}\right)$ as $S_{0}$ is varied. . . . . . . . . . . . . . . . 49

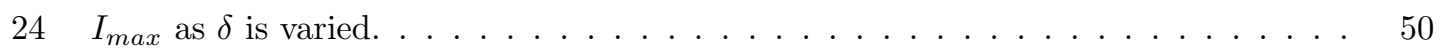

25 Contour Plot of All Possible Inter-Outbreak Periods . . . . . . . . . . . . . . . . 51

26 As in Figure $25,\left(\beta_{0}, \mu\right)=(1300,0.01) \ldots \ldots \ldots \ldots \ldots \ldots$

27 As in Figure $25,\left(\beta_{0}, \mu\right)=(500,0.05) \ldots \ldots \ldots \ldots \ldots$. . . . . . . . . 51

28 Plotting $S_{0}-P\left(S_{0}\right)$ as $S_{0}$ is varied for various values of $\delta \ldots \ldots$. . . . . 52

29 Plotting available periodic modes as $\delta$ varies . . . . . . . . . . . . . . . . . 54

30 As in Figure 29, but with the system parameters of measles. $\left(\beta_{0}, \gamma, \mu\right)=(478,28,0.02) 55$

31 Using the value of $\tilde{I}_{I I}$ at the midpoint of the inter-outbreak period as a proxy for the minimum value, plotted as $S_{0}$ is varied . . . . . . . . . . . 56

$32 j(t)=S(t) I(t)$ as $R_{0}$ is varied, with $S(0)=0.99 \ldots \ldots \ldots \ldots$

\section{List of Tables}

12 -norm of distance between $\gamma t$ and $\gamma \tilde{t}$ for varying $R_{0} \ldots \ldots . \ldots$ 


\section{Introduction}

Flu season comes once a year in North America. In the fall of each year, children return to school from their summer vacations, while university students may move from abroad. People spend more time indoors, together in enclosed spaces. Lack of sunlight leads to decreased levels of Vitamin D and thus weakened immune systems. There is no single known cause for the seasonality of the flu, but the aforementioned causes and countless more factors all contribute to the conclusion. Every year, the flu returns.

These events contribute to seasonal recurrence of disease in North America and other temperate regions, but other events such as migration of birds and periodic hatching of insects may contribute to similar seasonal outbreaks in other parts of the world.

While some viruses are little more than nuisances, some can change the world. Work on this thesis began in November 2019, shortly before the first reports of COVID-19 began in China. It may be noted that COVID-19 may be traced to a livestock market in the Wuhan province, and that such markets (as well as typical farmer's markets) may cause periodic variation in contact rate on a scale of weeks as opposed to the annual variation described previously. In an odd synchronicity, what was a topic chosen due to the author's personal interest in the application of mathematical models to biological problems came to the forefront of public consciousness. Policy decisions that have changed almost every aspect of public life have been made based on predictions made using mathematical models [4].

Attempts to predict the spread of infectious diseases using mathematical models can be traced back to the early Bernoulli's smallpox model in 1760, with the most influential work on the modern usage of compartmental models being that of Kermack and McKendrick [13]. The basis of the model is the hypothesis that the rate of infection is proportional to the population of infected as well as the population of susceptible individuals. This hypothesis led to the construction of what can be called the traditional "Susceptible-Infected-Removed" (SIR) model. The basic model is sufficient for the prediction of population dynamics during an outbreak, but it requires many simplifying assumptions that reduce the fidelity to real-world outbreaks.

Following the work of Kermack and McKendrick, research into the SIR model has focused on the reduction of assumptions. An immediate extension to said model is the introduction of "vital dynamics", which account for natural birth and death within the population, as seen in [8]. In this modified "SIR with vital dynamics" (referred to from now on as "V-SIR") model, more meaningful long-term predictions can be made. One key new state made possible by the introduction of vital 
dynamics is the "endemic" steady state, where the long-run state of the system includes a non-zero population of both susceptible and infected individuals, a state that is not possible in the basic SIR model.

Research into such epidemiological models has branched off in multiple directions as the field has matured, including the introduction of time delays, turning the model into a system of delaydifferential equations [18], the introduction of new states such as the "SEIR model" [16], that introduces a category for those who have been "exposed" to the infection but are not yet contagious. In addition, the system has been generalized into higher dimensional spaces through the consideration of multiple species with cross-infection. The real-world validity of such models has been tested as well $([7],[15])$, but no form yet mentioned was able to capture the behavior of seasonally recurring diseases, which appear to oscillate about the endemic equilibrium [10].

To capture the behavior of such seasonally recurring diseases, an oscillatory time dependence was introduced to the infection rate emulating the changes in societal conditions that may lead to seasonally recurring diseases [8]. This modification led to a new realm of possible dynamics. Damped oscillations had been observed in the solutions to the existing models with initial conditions near the endemic equilibrium; the introduction of an oscillation in the contact rate then leads to the possibility of resonance between the system's natural oscillations and the oscillation of the contact rate.

It has been rigorously proven [12] that for an SIR model with such a periodic variation in infection rate, there will exist a stable oscillatory mode with the same rate. Numerical simulations ([10], [19]) have shown that not only is there a periodic mode matching the rate of oscillation of the infection rate but that sub-harmonic modes are possible as well. These sub-harmonic modes exhibit a pattern: as the period of the oscillation increases, the severity of the outbreak increases as well. This phenomena has been observed through numerical simulation, but an analytical investigation has not been attempted.

The SIR model has had known analytic solutions since the work of Kermack and McKendrick [16], but the introduction of the periodic variation in the contact rate has complicated the system beyond being solvable with existing methods. The basic SIR model involves three inter-connected equations (which can be reduced to two, as will be seen later) and non-linearity. The seasonal SIR (S-SIR) model is further complicated by the inclusion of a time-varying infection rate, which has made algebraic analysis difficult.

The primary goal of this thesis is to continue the work done by Greenman, Kamo, and Boots [9]. Their work identified the resonance hierarchy of the S-SIR model by numerical simulation, while 
this work endeavors to advance understanding of the model by finding an analytic solution (or suitable approximation). This will enable us to study the changes in the qualitative behavior of the solutions as the system parameters are varied. 


\section{Mathematical Background}

This section is intended to provide an explanation of the necessary concepts applied in analyzing the problem of the S-SIR Model. It is assumed that the reader has an understanding of differential equations at the graduate level; this section is intended to extend then into the specific mathematical niches.

\subsection{Lambert $W$ Function}

The Lambert $W$ function [6], aka the "Product-Log" or "Omega" function is a special function arising from the multiple branches of the inverse of the function

$$
f(w)=w e^{w}
$$

It is defined in a way such that

$$
z=w e^{w}
$$

if and only if

$$
w=W_{k}(z)
$$

where $k$ is an integer. While the function is defined in the complex plane, when applied to the real numbers, only $W_{0}$, known as the principal branch, and $W_{-1}$ are needed. These branches are shown in Figure 1.

The $W$ function has particular application in this thesis, as the solution to equations of the form

$$
\xi=\alpha+\nu e^{\chi \xi}
$$

will be

$$
\xi=\alpha-\frac{1}{\chi} W\left(-\nu \chi e^{\alpha \chi}\right) .
$$

Equations of the form (4) will appear at several points, so the $W$ function will see application throughout this thesis.

\subsection{Asymptotic Relations}

Given functions $f(x), g(x)$, and the point $x_{0}$ within the domain of both, we may use the following definitions to describe the relationship between the behaviour of two functions in a particular limit. 


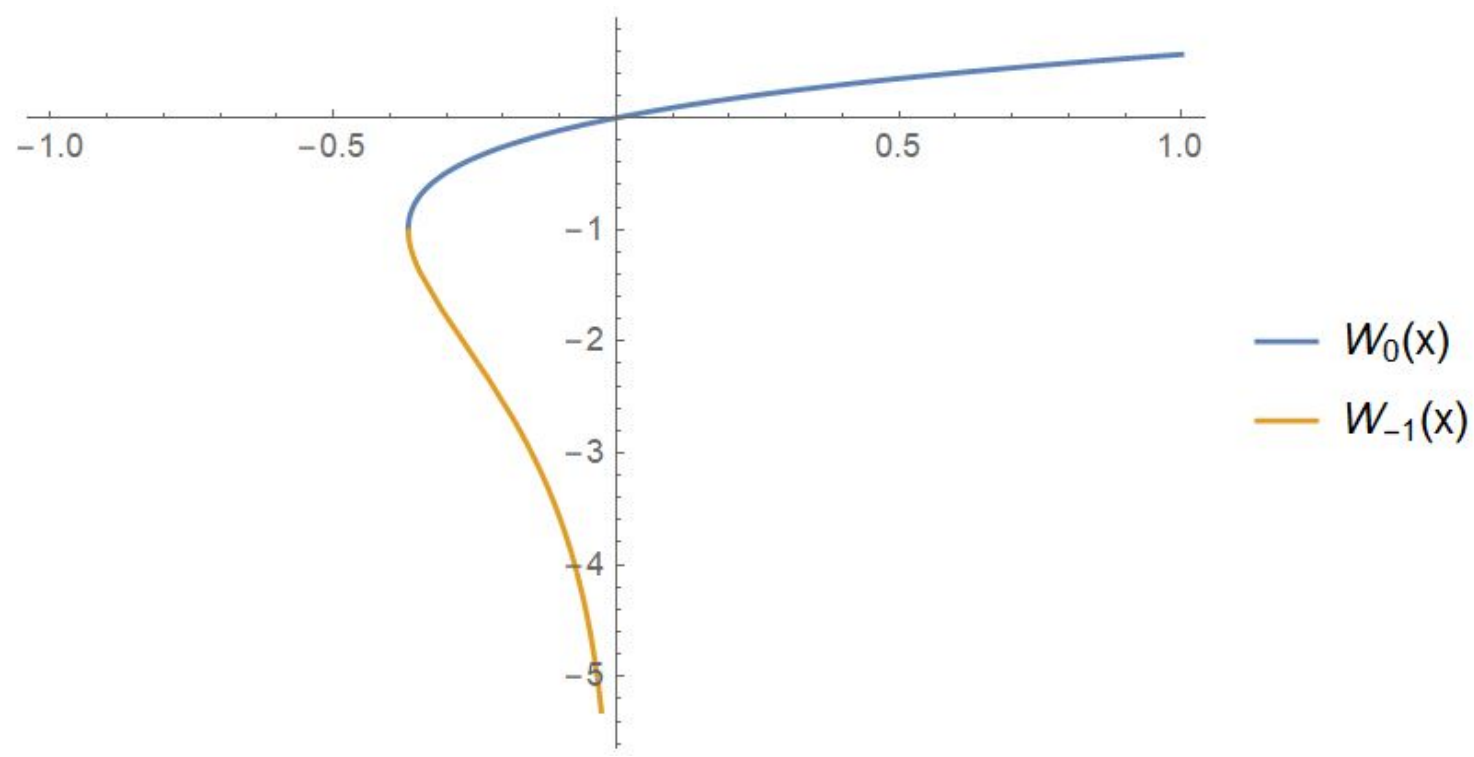

Figure 1: The principal branch of the Lambert $W$ function, as well as the branch $W_{-1}$. Note that $W_{0}$ is the branch of interest for the majority of its use here.

\section{Definition 1.}

$$
\text { If } \lim _{x \rightarrow x_{0}} \frac{f(x)}{g(x)}=0
$$

then $g(x)$ is asymptotically greater than $f(x)$, written as $g(x) \gg f(x), x \rightarrow x_{0}$. Additionally, we may say that $f(x)$ is asymptotically less than $g(x)$, written as $f(x) \ll g(x), x \rightarrow x_{0}$.

\section{Definition 2.}

$$
\text { If } \lim _{x \rightarrow x_{0}} \frac{f(x)}{g(x)}=1
$$

then $f(x)$ is asymptotically equal to $g(x)$, written as $g(x) \sim f(x), x \rightarrow x_{0}$.

Having defined the concept of such asymptotic relations, we can also discuss what are called "asymptotic series". Given a function $f(x)$, we may express it in the form

$$
f(x) \sim \sum_{n=0}^{\infty} \phi_{n}(x), \quad x \rightarrow x_{0}
$$

where $\quad \phi_{n}(x) \gg \phi_{n+1}(x), \quad x \rightarrow x_{0}, \quad$ for all $n$.

Each function in the sequence $\left\{\phi_{n}\right\}$ is asymptotically larger than the subsequent term, and thus

$$
\begin{aligned}
f(x) & \sim \phi_{0}(x), \quad x \rightarrow x_{0} \\
\text { and } \quad f(x) & \sim \phi_{0}(x)+\phi_{1}(x), \quad x \rightarrow x_{0}, \\
\text { and } \quad f(x) & \sim \phi_{0}(x)+\phi_{1}(x)+\phi_{2}(x), \quad x \rightarrow x_{0}
\end{aligned}
$$

Note that as long as the above requirements are satisfied, the asymptotic series need not converge. 


\subsection{Dominant Balance}

The method of dominant balance is in essence an algorithm for creating approximate solutions to a system of equations. The steps are as follows:

1. Make an assumption regarding the relationship of terms. For example, "Term $1 \sim$ Term 2 $\gg$ Term 3".

2. Solve the resulting relation as though it were an equation. In the above case, one would solve the equation "Term $1=$ Term 2 "

3. Having an approximate solution, verify that the assumptions made in Step 1 are valid for the result. 


\section{Application Background}

This section is intended to provide the necessary background information regarding the mathematical model of interest in this thesis, specifically to the "SIR" model of the spread of infectious diseases. For the purposes of this thesis, the primary focus is on the possible outcomes of the model taken on face value, as opposed to whether the model itself is necessarily valid. The realism of the situations investigated is not a question that has been considered, merely whether or not the phenomena observed are internally consistent with the mathematical model.

As mentioned in the introduction, the mathematical modeling of epidemic spread was pioneered by Kermack and McKendrick [13]. As such, the presentation below may be considered to be paraphrasing their work.

\subsection{The SIR Model}

Consider a population in which a single infected individual has been introduced. If we assume that all members of the population who are not infected are susceptible to infection, we may create two compartments, susceptible and infected, designated by $S(t)$ and $I(t)$, respectively.

Without access to significant time and computational power, creating a model which attempts to deterministically describe the motion of populations and the process of infection is not a viable option, and so we choose to make the following simplifying assumptions: as individuals go about their lives, there is a particular rate at which susceptible individuals will encounter infected individuals. Additionally, we assume that infection occurs with a particular probability. These two factors are combined to give the infection rate, $\beta$.

We may also assume that the rate at which individuals are infected is proportional to the number of both infected and susceptible, such that $S^{\prime}(t) \propto-I(t) S(t)$, and $I^{\prime}(t) \propto I(t) S(t)$, where the constant of proportionality will be named $\beta$.

Once an individual is infected, they will either recover or be euphemistically "removed" from the system. For the basic system at hand, we assume that recovery confers complete immunity to the disease. Thanks to the euphemistic wording, we may consider both recovered individuals and those who die from the disease as transitioning into the "removed" category, as they will no longer influence the dynamics of the system. This introduces a new system parameter, $\gamma$, called the rate of removal, and a new compartment, $R(t)$, for those who die or recover from the disease. We further assume that the actual change in the number of infected is dependent on both $\gamma$ and the 
number of infected themselves.

It must be noted that "recovered" and "removed" are interchangeable for the purposes of this basic model, though this is not necessarily the case in general.

Having constructed this model conceptually, we may quantify it using the following system of equations

$$
\begin{aligned}
S^{\prime}(t) & =-\beta I(t) S(t) \\
I^{\prime}(t) & =\beta I(t) S(t)-\gamma I(t) \\
R^{\prime}(t) & =\gamma I(t),
\end{aligned}
$$

where $I(t), S(t), R(t) \geq 0$ and $\beta, \gamma>0$.

We may see that $S^{\prime}+I^{\prime}+R^{\prime}=0$, and thus it must be that

$$
S(t)+I(t)+R(t)=N,
$$

where $N$ is the total population. Having this, it is possible to fully describe the system in terms of only two of the compartments. We are free to choose the compartments considered as is useful for the particular situation.

Of particular interest in the analysis of this model is the "epidemic threshold". Note that for any contextually valid values of any parameter, $S^{\prime}(t) \leq 0$ and $R^{\prime}(t) \geq 0$. For $I^{\prime}(t)$, however, if $S(t)<\frac{\gamma}{\beta}$ then $I^{\prime}(t)<0$. The existence of a turning point in the infected population is contextually unsurprising, but it is worth noting that this also implies a threshold below which no true epidemic is possible. The quantity $R_{0}=\frac{\beta}{\gamma}$ (also known as the basic reproduction number), is commonly used to understand the threat of epidemic that a disease poses, as it can be understood as the number of individuals a single infected individual will infect. If $R_{0}<1$, then the infected are recovering (or perishing) faster than they are infecting others, leading to the disease dying out. The disease may still spread, but the population is inevitably drawn towards the equilibrium line at $(S, I, R)=(x, 0, N-x)$, for $x \leq N$. Conversely, if $R_{0}>1$, then the disease will spread more rapidly than individuals recover, leading to an epidemic.

An example of the initial development of the system is shown in Figure 2. Note that as time continues, the system remains in the equilibrium $(S, I, R)=(0,0,1)$. 


\section{Population}

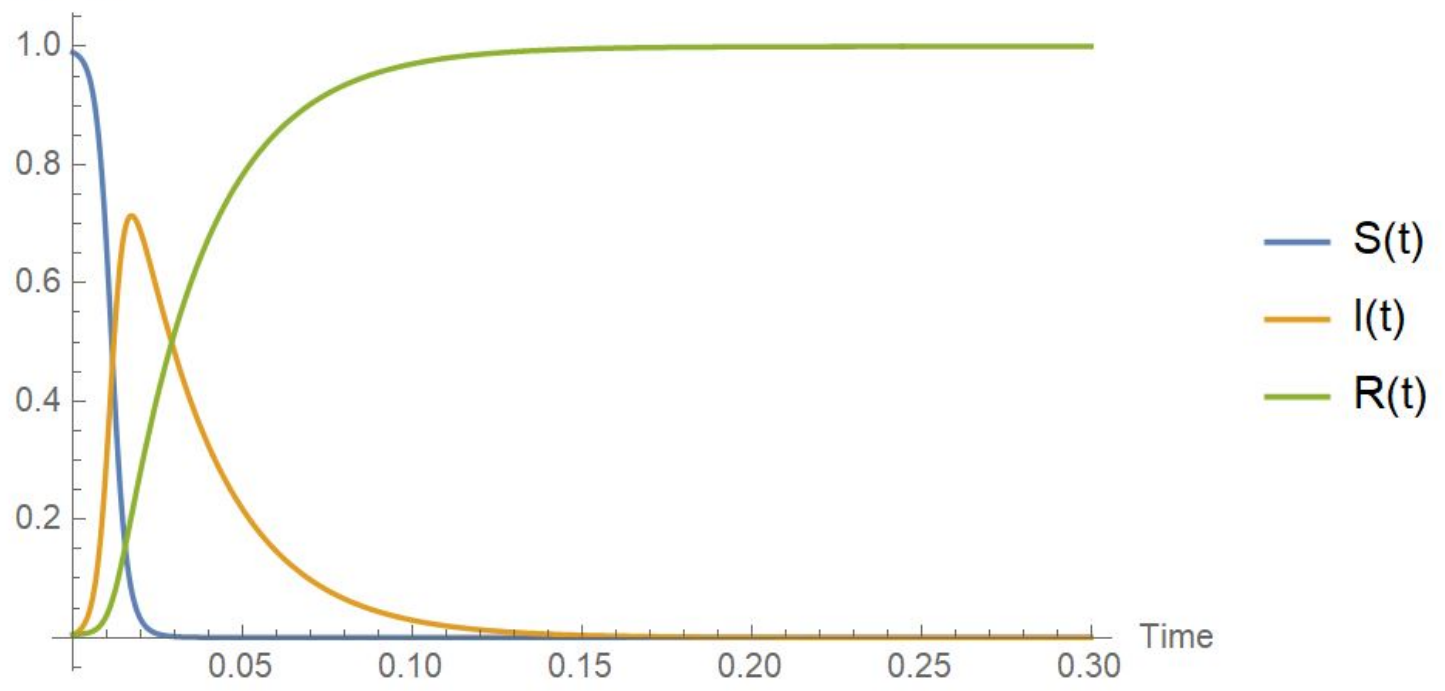

Figure 2: Initial development of the SIR model for $\left(\beta_{0}, \gamma\right)=(500,40)$ and $(S(0), I(0))=$ $(0.99,0.005)$

\subsection{The Vital SIR (V-SIR) Model}

At the time scales of a typical disease outbreak, considerations of natural birth and death rates are not necessary. As time scales are increased, however, these must be considered. For the purposes of the model examined in this thesis, assume that the natural rates of birth and death are equal, labelled by $\mu$, and that every individual in the population is equally capable of reproduction regardless of infection. This produces the modified system of equations

$$
\begin{aligned}
S^{\prime}(t) & =N \mu-\mu S(t)-\beta I(t) S(t) \\
I^{\prime}(t) & =\beta I(t) S(t)-(\gamma+\mu) I(t) \\
R^{\prime}(t) & =\gamma I(t)-\mu R(t) .
\end{aligned}
$$

We must note that if the rates of birth rate and death rate were unequal, then the population will no longer be constant, leading either to total extinction of the population or growth beyond the normalized value.

Letting $\lambda=\gamma+\mu$ and $\phi=N \beta-\lambda$, we can see that this system has an equilibrium point at

$$
S_{E}=\frac{\lambda}{\beta}, \quad I_{E}=\frac{\mu \phi}{\beta \lambda}, \quad R_{E}=\frac{\gamma \phi}{\beta \lambda} .
$$

If $\phi>0$, this is an equilibrium known as the endemic equilibrium point. Note that the Jacobian matrix of the system formed by equations 10 and 11 is given by

$$
A=\left[\begin{array}{cc}
-\mu-\beta I(t) & -\beta S(t) \\
\beta I(t) & \beta S(t)-\lambda
\end{array}\right]
$$




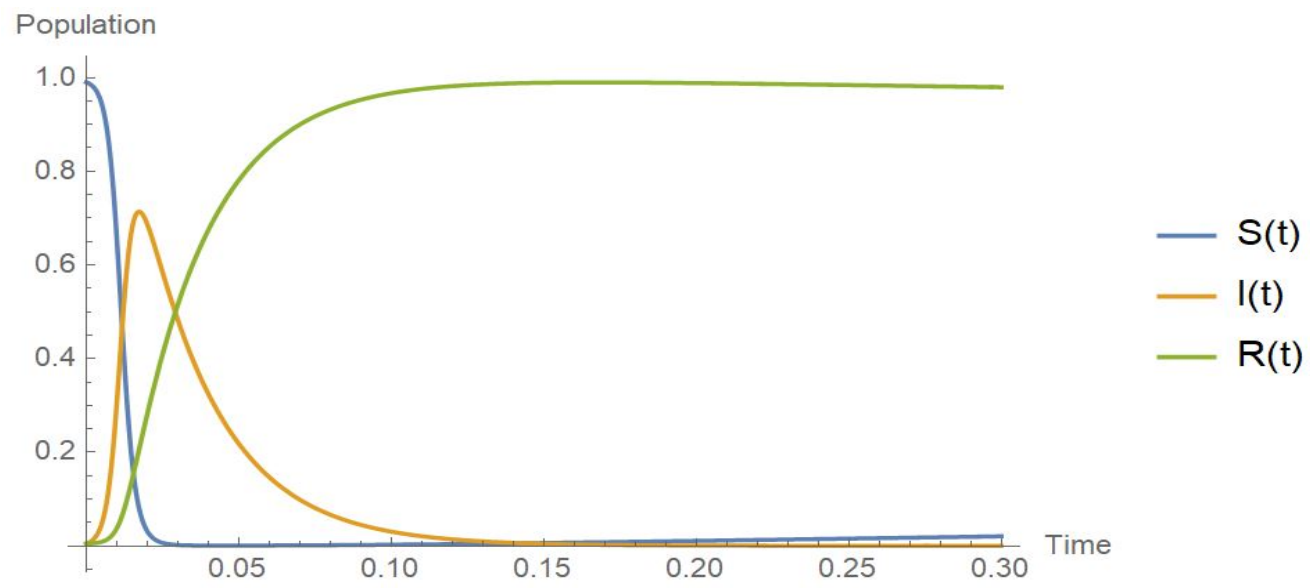

(a) Outbreak trajectory of the V-SIR model for $\left(\beta_{0}, \gamma, \mu\right)=(500,40,0.1)$, initial conditions as in Figure 2 Population

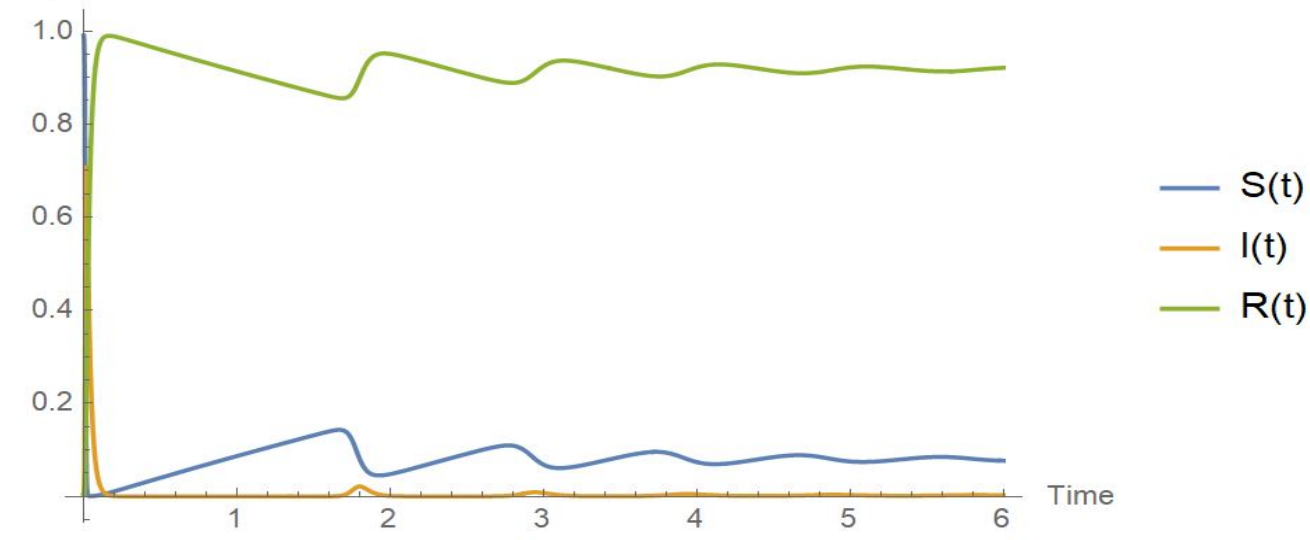

(b) Long run development of the V-SIR model, parameters as above

Figure 3: V-SIR solution development over varying time scales. Note that the initial development is nearly identical to that of the traditional SIR shown in Figure 1.

Taking $S(t)=S_{E}$ and $I(t)=I_{E}$, we find the eigenvalues to be

$$
\begin{aligned}
& \Lambda_{1}=\frac{-\beta \mu n-\sqrt{\mu} \sqrt{4 \lambda^{3}+N^{2} \beta^{2} \mu-4 N \beta \lambda^{2}}}{2 \lambda} \\
& \Lambda_{2}=\frac{-\beta \mu n+\sqrt{\mu} \sqrt{4 \lambda^{3}+N^{2} \beta^{2} \mu-4 N \beta \lambda^{2}}}{2 \lambda} .
\end{aligned}
$$

For $\lambda>0, \beta>\lambda, N>\frac{\lambda}{\beta}$, and $0<\mu<\left(4 N \beta \lambda^{2}-4 \lambda^{3}\right) N^{-2} \beta^{-2}$, we find both eigenvalues to have negative real parts, so the equilibrium will be stable whenever it exists. The development of the system into this point is shown in Figure 3. Note that the initial development of the system is nearly identical to that shown for the SIR model in Figure 2.

\subsection{The Seasonal SIR (S-SIR) Model}

As mentioned in the introduction, there are various factors which may cause the seasonal recurrence of a disease. For the purpose of this thesis, we assume that it is the $\beta$ value that varies, and that it 


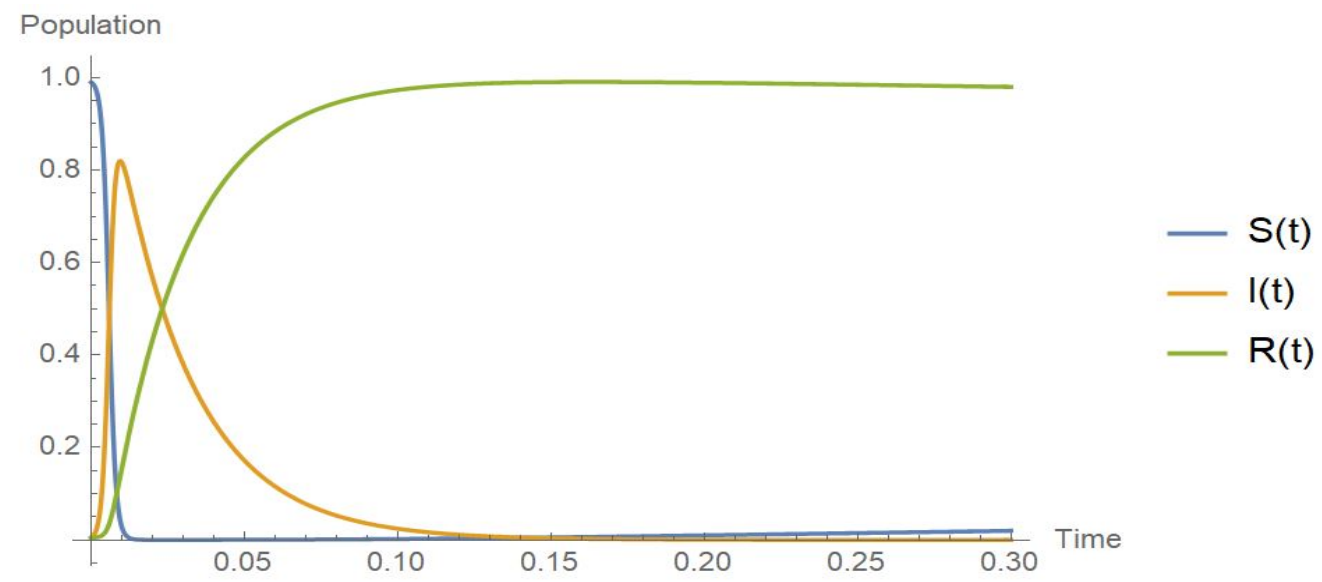

(a) Outbreak trajectory of the S-SIR model for $\left(\beta_{0}, \delta, \gamma, \mu\right)=(500,0.9,40,0.1)$, initial conditions as in Figure 2

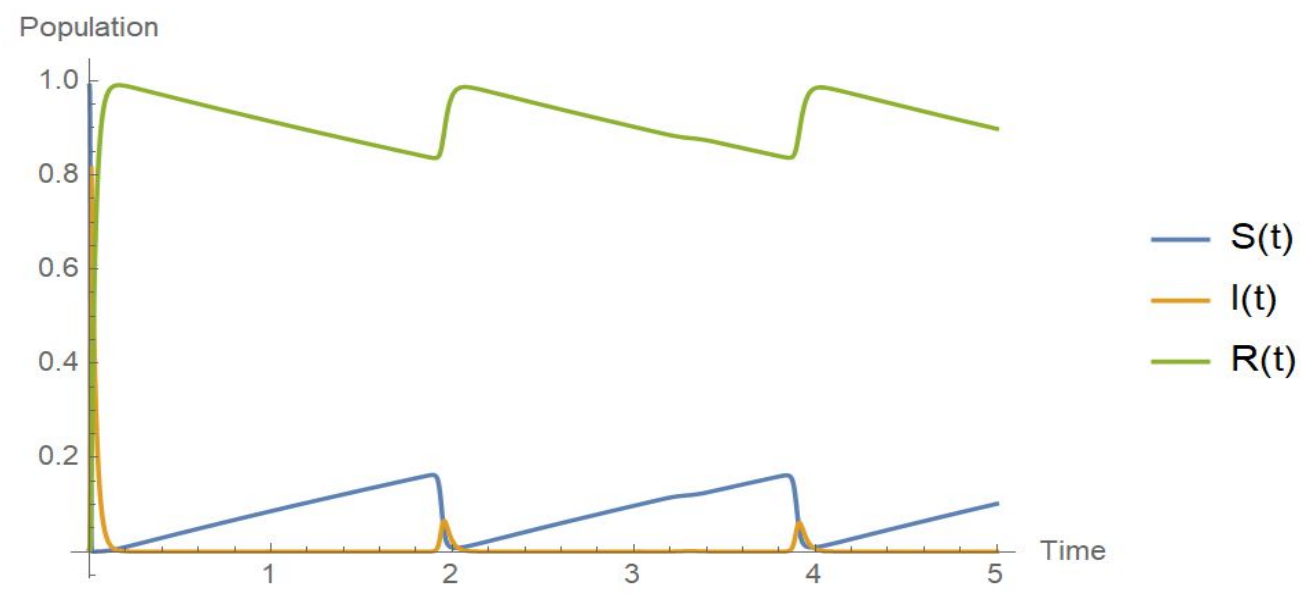

(b) Long run development of the S-SIR model, parameters as above

Figure 4: S-SIR solution development over varying time scales. Note that as in Figure 2a, the initial outbreak is nearly identical to that shown in Figure 1. In figure (b) above, note the periodic outbreaks. "Mini-outbreaks" appear to occur at off-integer time values, but major outbreaks appear to occur only at (or near) integer t values.

does so sinusoidally, such that $\beta(t)=\beta_{0}(1+\delta \cos 2 \pi t)$, where $\delta$ is referred to as the "seasonality", resulting in the system

$$
\begin{aligned}
S^{\prime}(t) & =N \mu-\mu S(t)-\beta_{0}(1+\delta \cos 2 \pi t) I(t) S(t) \\
I^{\prime}(t) & =\beta_{0}(1+\delta \cos 2 \pi t) I(t) S(t)-(\gamma+\mu) I(t) \\
R^{\prime}(t) & =\gamma I(t)-\mu R(t) .
\end{aligned}
$$

A numerical solution to this system is demonstrated in Figure 4.

For the remainder of theis thesis, assume that, unless otherwise stated, the population is normalized such that $N=1$. 


\section{Numerical Simulations and the Guiding Question}

To begin understanding the behaviour of the solution to the S-SIR model, we first attempt numerical simulations. It must be noted that, due to the dramatically varying time scales, the model proves difficult for some numerical ODE solvers to deal with. Wolfram Mathematica has been chosen for all numerical simulations within, particularly for the "Stiffness-Switching" method implemented into its "NDSolve" function. This allows Mathematica to automatically determine the appropriate numerical solution method and switch the method when stiffness is encountered. Additionally, in all simulations, the "Accuracy Goal" option was set to $\infty$, instructing Mathematica to use relative error as the basis for error control exclusively.

To begin the numerical investigation of the model, system parameters have been chosen based on the paper by Greenman et al. [9]. Fixing the system parameters $(\beta, \gamma, \mu)=(500,40,0.01)$, setting initial conditions to $\left(S_{0}, I_{0}, R_{0}\right)=(0.99,0.01,0)$, and varying $\delta$ reveals several different possible trajectories for the system to take. Figure 5 illustrates three different qualitative forms that the $S(t)$ component of solutions may take. Within a relatively narrow region of variation, three solutions with significantly different qualitative forms are found, corresponding to solutions with period 1,3 , and 10 .

The phenomenon which inspired this work is the pattern visible: as the inter-outbreak period increases, the curve for $S(t)$ approaches a "saw-tooth"-like profile. In the limit as the period grows large, the severity of the outbreak increases as well. This behavior led to the guiding question for this thesis: why does an outbreak happen when it does?

Examining the profile of the $I(t)$ component of these long inter-outbreak period solutions shows the peculiar behavior most prominently: as shown in Figure 6a, the infected population becomes nearly zero in the inter-outbreak period, then seems to inexplicably shoot up, taking a shape resembling a Dirac $\delta$ function. Note that the period of the forcing is unchanged: in all solutions shown, the seasonal forcing is occurring with period 1. The only parameter being varied is the value of $\delta$, the strength of the seasonal forcing.

Qualitatively, it is visible that there are two distinct regions in the long inter-outbreak period solutions: the inter-outbreak region, where $S(t)$ appears linear and $I(t)$ appears to be close to zero, and the outbreak region, where $I(t)$ rapidly increases and falls again, and where $S(t)$ rapidly falls. In the current form, the plot for $I(t)$ gives very little useful information. Logarithmically scaling the vertical axis reveals the behavior of $I(t)$ more meaningfully and reveals a hint towards what is causing the outbreaks when they occur. 


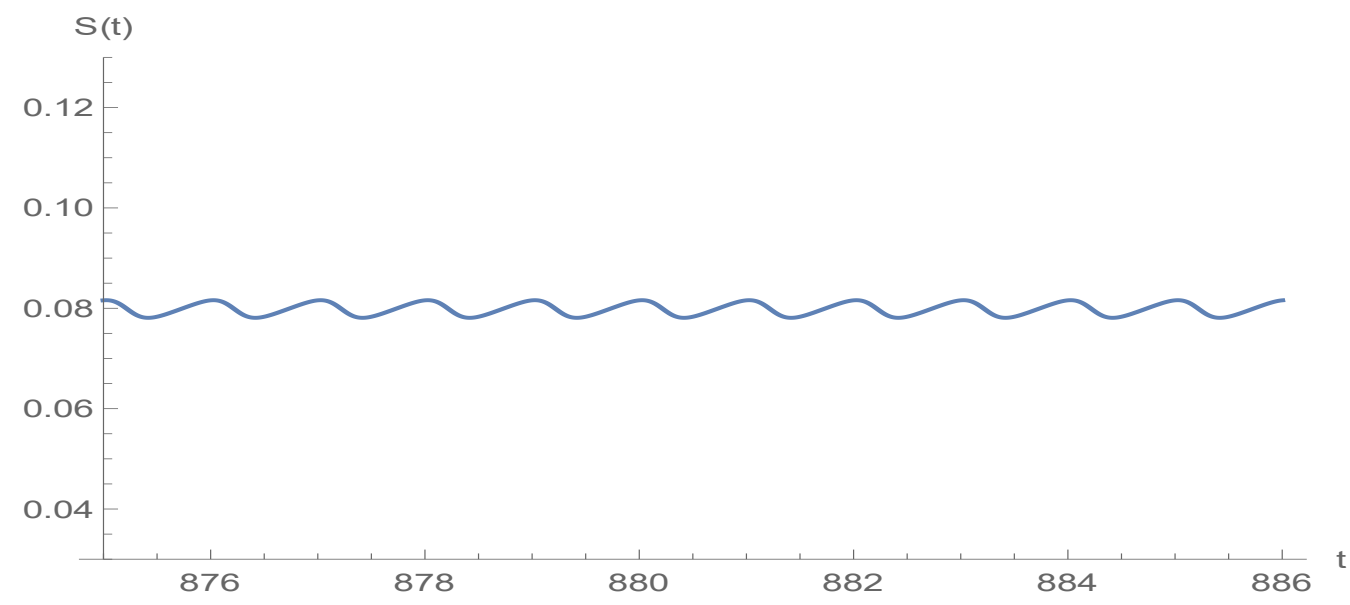

(a) $\delta=0.2$

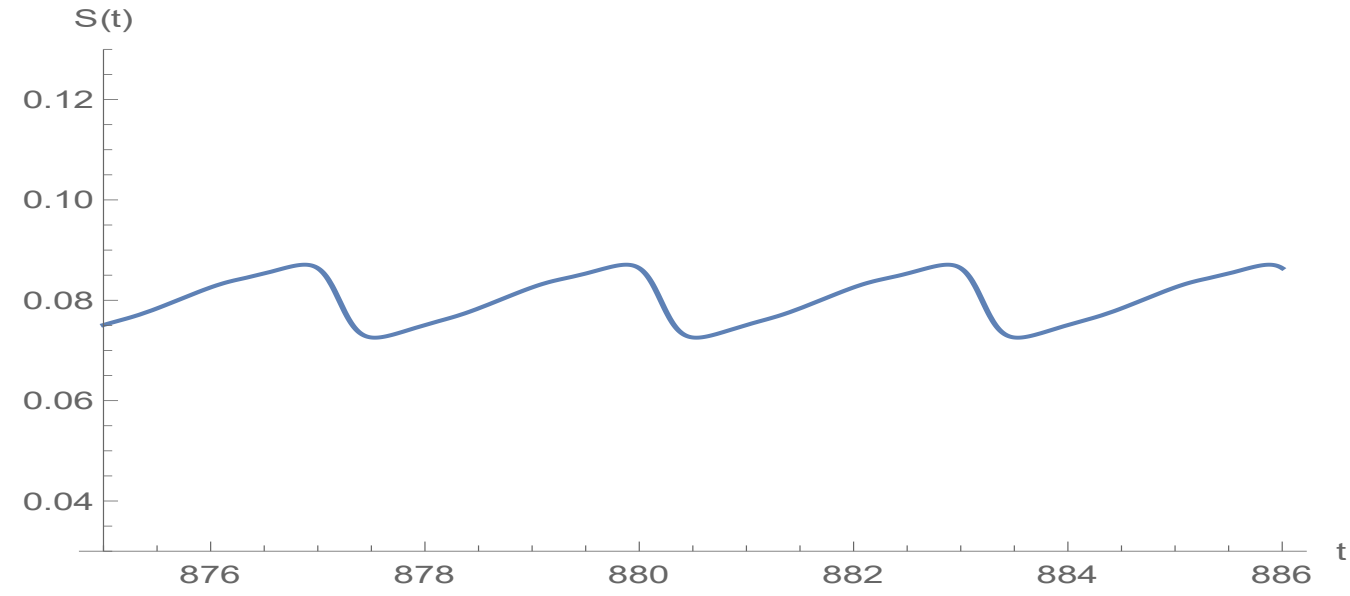

(b) $\delta=0.1$

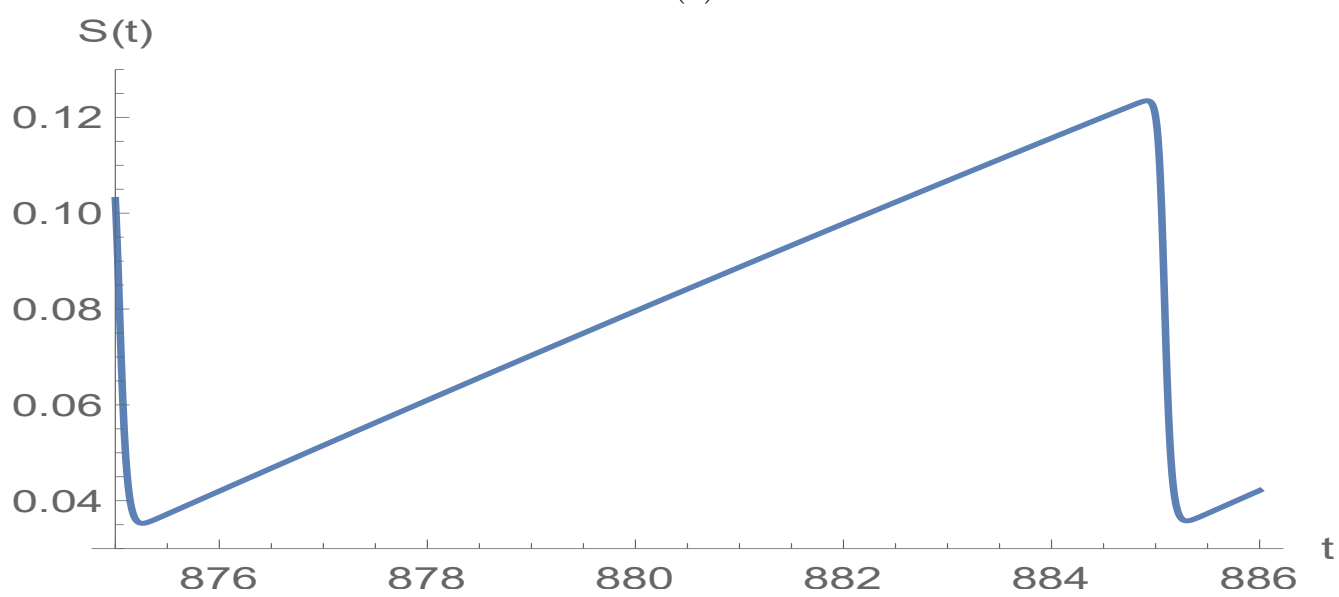

(c) $\delta=0.21$

Figure 5: Three different possible trajectories for $S(t)$ over time 
Susceptible Population

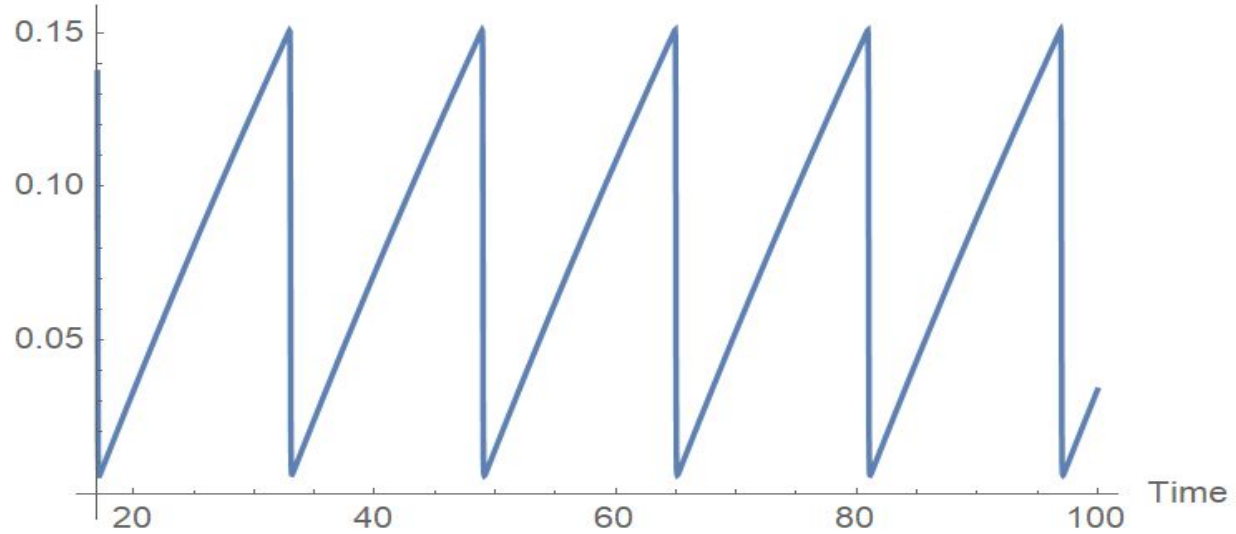

(a) $I(t)$ for $\delta=0.9$

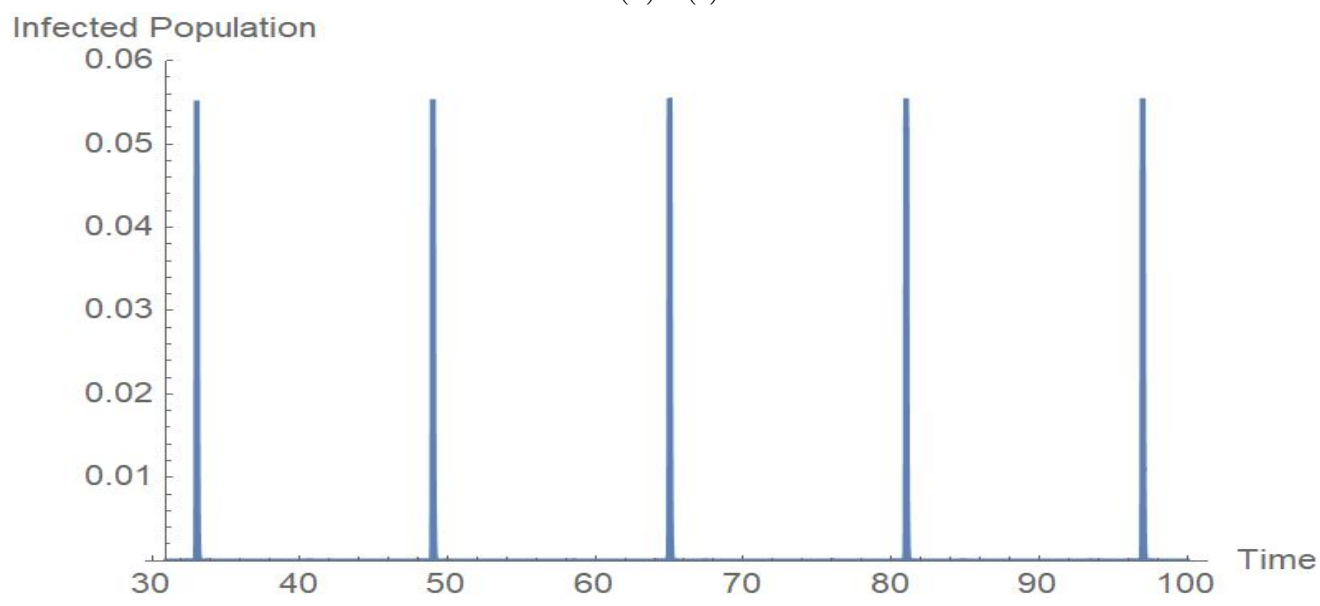

(b) $S(t)$ for $\delta=0.9$

Figure 6: Period 16 Mode

Susceptible Population

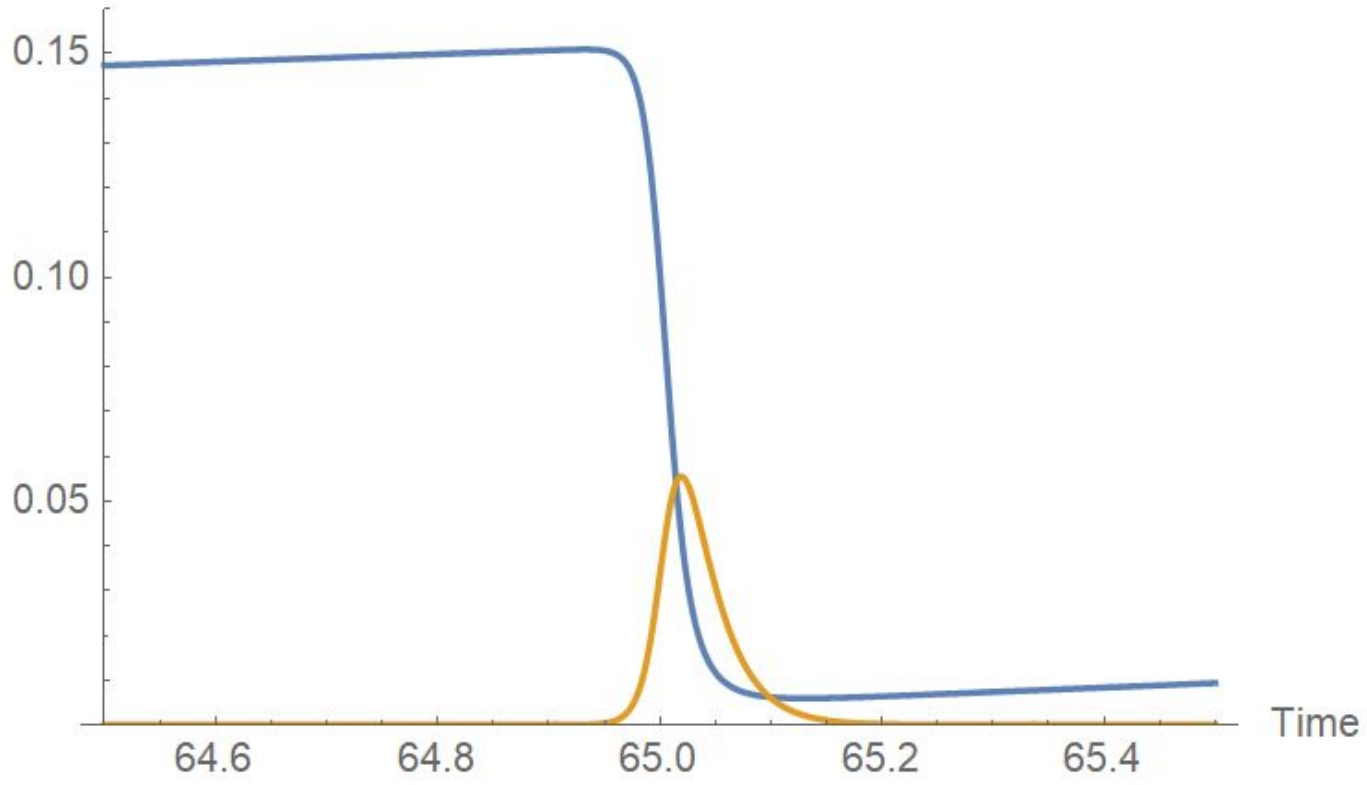

Figure 7: Close-Up on Outbreak region in Figure 6. $\left(\beta_{0}, \delta, \gamma, \mu\right)=(500,0.9,40,0.01)$ 


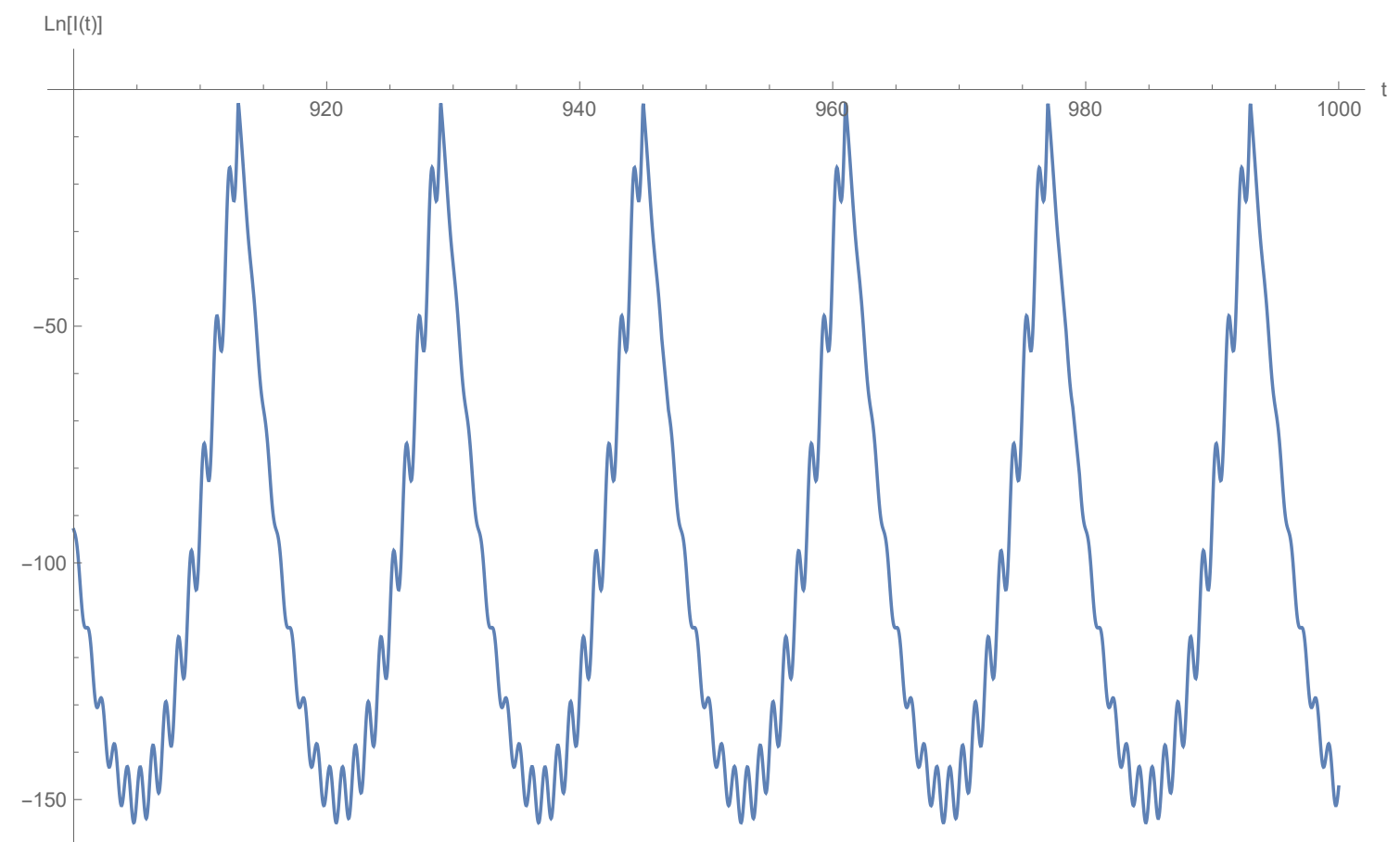

Figure 8: Logarithmically scaled $I(t)$ for the period 16 mode, $\left(\beta_{0}, \delta, \gamma, \mu\right)=(500,0.9,40,0.01)$

Figure 8 reveals a relatively comprehensible trajectory, appearing to be a superposition of longperiod and short-period sinusoidal oscillations, where it appears that the severity of the short period oscillation reaches a maximum toward the middle of the region. Additionally, we can see that the short-period oscillation matches the period of the seasonal forcing: this reflects the behaviour described in Section 3.3. As $\beta(t)$ varies, the endemic equilibrium point varies.

The fact that the behavior in the logarithmically scaled region is comprehensible gives a significant clue that allows investigation of this inter-outbreak region: it appears that for some function $J(t)$, we may apply the transformation $I(t)=e^{J(t)}$. This transformation will prove crucial when attempting to construct the approximate solutions for the inter-outbreak region.

In pursuit of the goal of understanding why an outbreak occurs when it does in the S-SIR model, we may now see that it is clearly dictated by the long-run oscillatory behavior of $\ln I$. In order to understand analytically how the system parameters will change the system's qualitative behavior, it is primarily the behaviour shown in the inter-outbreak region that we seek to understand and approximate analytically. 


\section{Construction of Approximate Solutions}

Now that the expected qualitative behaviour of the long-period modes has been found, it is possible to begin the process of finding local approximations to the solution to S-SIR model in the interoutbreak and outbreak regions.

Unless indicated otherwise, all numerical simulations and plots shown are generated with the parameter set

$$
\left(\beta_{0}, \delta, \gamma, \mu, S(0), I(0)\right)=(500,0.9,40,0.01,0.99,0.01)
$$

This arises from the phenomena observed in Section 4, as they resulted in one of the longest period modes observed in the initial experimentation phase of research.

\section{$5.1 \quad$ Notation}

Before proceeding, some notation must be established. For the discussion at hand, each orbit of the population will be separated into two regions: the "outbreak" region, which will be labeled as Region I, and the "inter-outbreak" region, which will be labeled Region II. Locally, Region I is considered to start at $t=t_{0}$ and end at $t=t_{1}$, and Region II is considered to begin at $t=t_{1}$ and end at $t=t_{2}$. When the relationship between multiple orbits must be discussed, a super-script index will be introduced, such that $t_{0}^{n}$ is the beginning of the $n^{\text {th }}$ outbreak. In this notation, note that we may state $t_{2}^{n}=t_{0}^{n+1}$ and $t_{0}^{n}=t_{2}^{n-1}$. Unless otherwise stated, the superscript will be suppressed for readability.

To reduce ambiguity, the tilde accent, " $~ "$ will be used to designate approximate values as opposed to full solution values, such that we will be finding $\tilde{S}(t)$ and $\tilde{I}(t)$ in this section.

When it becomes necessary to discuss the trajectory of the whole system in the $S$ - $I$ plane, we will use the vector

$$
E(t)=\left(\begin{array}{c}
S(t) \\
I(t)
\end{array}\right)
$$

to discuss the trajectory in the plane.

With this notation established, the piece-wise approximation that will be found in this section may 
be written as

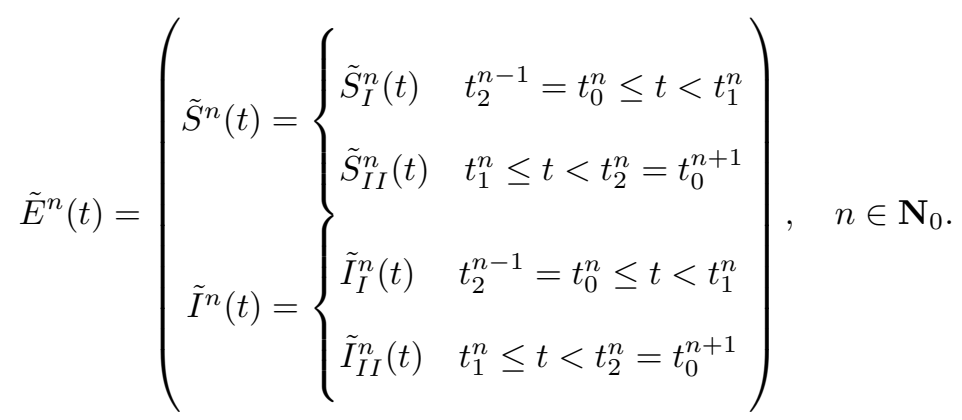

To begin constructing the approximate solutions, we will start in Region (II), as the derivation there is much more straightforward than what will be required in Region (I).

\subsection{Inter-Outbreak Region (II)}

\subsubsection{Susceptible}

The numerical simulations indicate that $I(t)$ becomes very small in the inter-outbreak region. We begin our attempt at finding an approximate solution by way of a dominant balance argument, using the assumption that

$$
S^{\prime}(t) \sim \mu-\mu S(t) \gg \beta(t) S(t) I(t) .
$$

This reduces equation (10) to a simple linear equation in $S(t)$, having solution

$$
S(t)=1-c_{1} e^{-\mu t}
$$

When the initial condition $S\left(t_{1}\right)=S_{1}$ is applied, the approximate solution for this region

$$
\tilde{S}_{\mathrm{II}}(t)=1+\left(S_{1}-1\right) e^{-\mu\left(t-t_{1}\right)} .
$$

is found. This doesn't immediately appear to have the expected behaviour (Figure $6 \mathrm{~b}$ seems to indicate a linear shape), but it is worth remembering that $\mu$ is very small in parameter sets examined so far, such that $S(t)$ is locally linear even over the scale of 16 time units.

\subsubsection{Infected}

We begin with the transformation $I(t)=e^{J(t)}$. We may substitute this expression into equation (11):

$$
e^{J(t)} J^{\prime}(t)=\beta_{0}(1+\delta \cos (2 \pi t)) e^{J(t)} S(t)-\lambda e^{J(t)} .
$$


Dividing through by $e^{J}(t)$, we can see that the exponential transformation serves an important role: the equation is now linear with respect to $J(t)$. Including the approximation for $S(t)$ from equation (23), the equation becomes directly integrable:

$$
J^{\prime}(t)=\beta_{0}(1+\delta \cos (2 \pi t))\left(1-\left(1-S_{1}\right) e^{-\mu\left(t-t_{1}\right)}\right)-\lambda .
$$

While this is a nearly miraculously simple equation, the solution itself is a labyrinth of terms. To make the solution comprehensible, we shall include the initial condition $I\left(t_{1}\right)=I_{1}$ and fold together existing parameters as follows:

Let

$$
\begin{gathered}
b=\frac{\beta_{0} \delta}{4 \pi^{2}+\mu^{2}}, \\
\hat{S}(t)=\left(S_{1}-1\right) e^{-\mu\left(t-t_{1}\right)}, \\
c=\beta_{0} \delta\left(\frac{\left(S_{1}-1\right)}{\mu \delta}+\frac{\left(S_{1}-1\right) \cos 2 \pi t_{1}}{4 \pi^{2}+\mu^{2}}-\frac{\left(4 \pi^{2} S_{1}+\mu^{2}\right) \sin 2 \pi t_{1}}{2 \pi\left(4 \pi^{2}+\mu^{2}\right)}\right) .
\end{gathered}
$$

This results in the solution

$$
J(t)=\ln \left(I_{1}\right)+c+\phi\left(t-t_{1}\right)+\frac{\beta_{0} \delta \sin (2 \pi t)}{2 \pi}-\hat{S}(t)\left[\frac{\beta_{0}}{\mu}+b(\mu \cos 2 \pi t-2 \pi \sin 2 \pi t)\right],
$$

which then indicates that the approximate solution for $\tilde{I}(t)$ in the inter-outbreak region is given by

$$
\tilde{I}_{\mathrm{II}}(t)=I_{1} \exp \left(c+\phi t+\frac{\beta_{0} \delta \sin (2 \pi t)}{2 \pi}-\hat{S}(t)\left[\frac{\beta_{0}}{\mu}+b(\mu \cos (2 \pi t)-2 \pi \sin (2 \pi t))\right]\right) .
$$

Plotted in Figure 9 is a comparison of a numerical solution and the approximate solution for the inter-outbreak period. We can note that though the two curves do not coincide exactly, the main qualitative details of the curve are preserved. As expected, the two curves deviate at the end point, corresponding to the time of outbreak. Additionally, we may see that the approximate solution will be crossing the $\ln I=0$ line at precisely $t=16$, while it deviates from the numerical solution at a time very slightly beforehand.

We must also note that from the form of equation (26), the curve will vary smoothly as the system parameters are varied. There is no necessary requirement that the curve crosses the $\ln I=0$ line at integer time values. Due to the oscillatory nature of the curve, however, we may see that, as shown in Figure 10, there is a limited range about integer values of $t$ where it is possible for an outbreak to occur. In the case shown, we may see that the middle of the interval between $t=15$ and $t=16$ cannot contain any outbreaks. Combining this with the fact that the numerics suggest that outbreaks may only occur at near-integer $t$ values, we will henceforth round the time of outbreak to the nearest integer. 


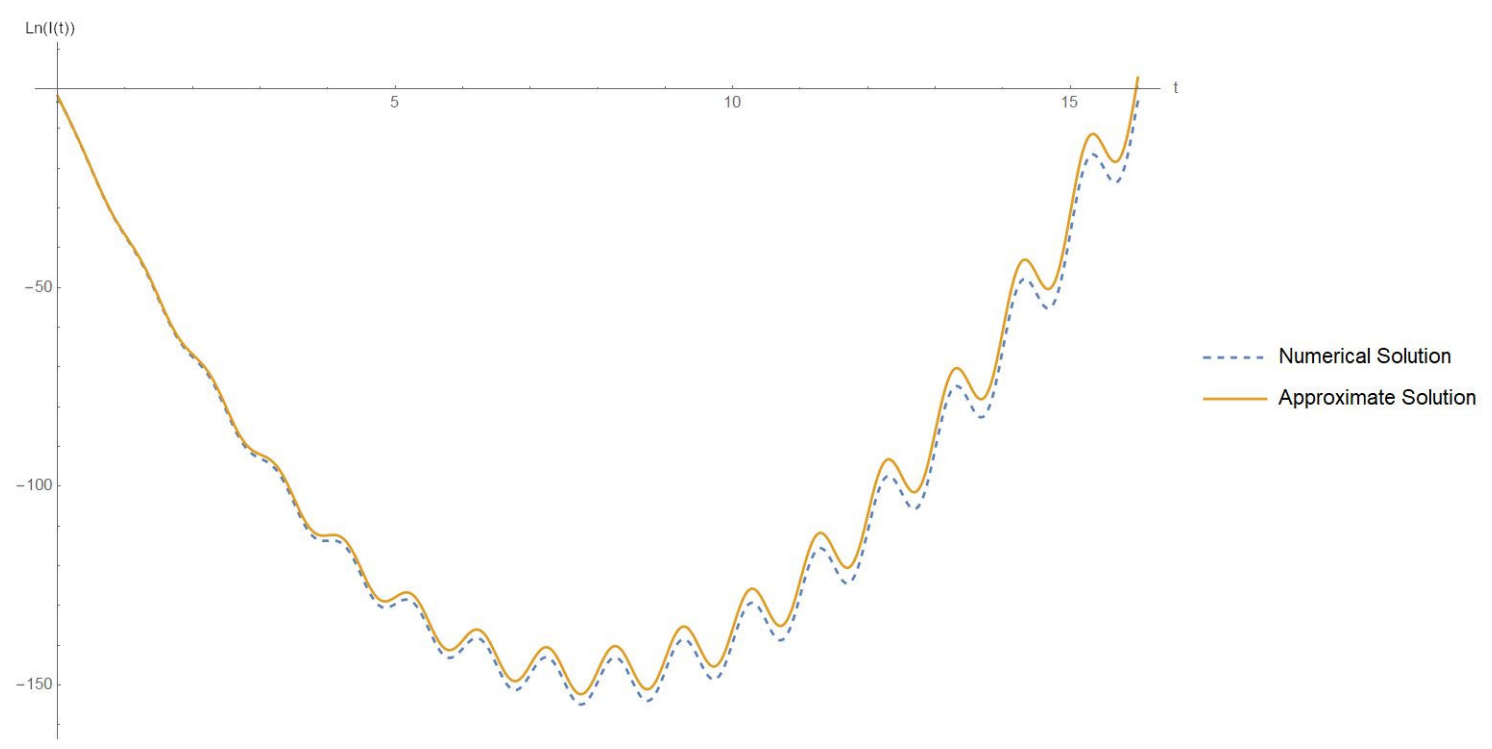

Figure 9: Comparing $\ln I(t)$ obtained by numerical solution vs. using the approximate solution.

\subsubsection{Dominant Balance Confirmation}

To discuss the validity of the dominant balance arguments made, the domain being considered is significant. $\tilde{I}_{\mathrm{II}}(t)$ as described above is an exponential function, which contains constant, linear, sinusoidal, exponential, and exponential multiplied by sinusoidal terms within its argument. Figure 11 shows the vastly different scales of $\tilde{S}_{I I}(t)$ and $\tilde{I}_{\mathrm{II}}(t)$ over Region (II), where the two approximate solutions were plotted using the following parameters:

$$
\left(\beta_{0}, \delta, \gamma, \mu, S_{1}, I_{1}, t_{1}\right)=\left(500,0.9,40,0.01,6.36 \times 10^{-3}, 1.54 \times 10^{-4}, 0\right) .
$$

The figure was logarithmically scaled for comprehensibility, as otherwise the fine detail dynamics were not visible when plotted over the whole time scale. As can be seen, $\tilde{S}_{I I}(t)$ spends most of its time being orders of magnitude larger that $\tilde{I}_{\mathrm{II}}(t)$, validating the dominant balance argument made, as well as agreeing with the numerical results observed in Section 4. Equally visible, and not unexpected, is the fact that the two populations are on similar scales at the end points of the region plotted, corresponding to the outbreak regions.

For a more rigorous analytical approach, let the outbreak end of the previous outbreak be $t=0$, and let $t_{c}$ and $p$ be real numbers such that $0<t_{c}<p$, where $p$ is the length of the inter-outbreak period and $t_{c}$ is an arbitrary point between outbreaks.

To begin, we consider the behaviour of $\tilde{I}_{\mathrm{II}}(t)$ from equation (26). The sinusoidal oscillating terms may prove troublesome, but we may first note that

$$
\frac{\beta_{0}}{\mu}+b(2 \pi-\mu)<\frac{\beta_{0}}{\mu}+b\left(\mu \cos \left(2 \pi t_{1}\right)-2 \pi \sin \left(2 \pi t_{1}\right)\right)<\frac{\beta_{0}}{\mu}+b(2 \pi+\mu) .
$$




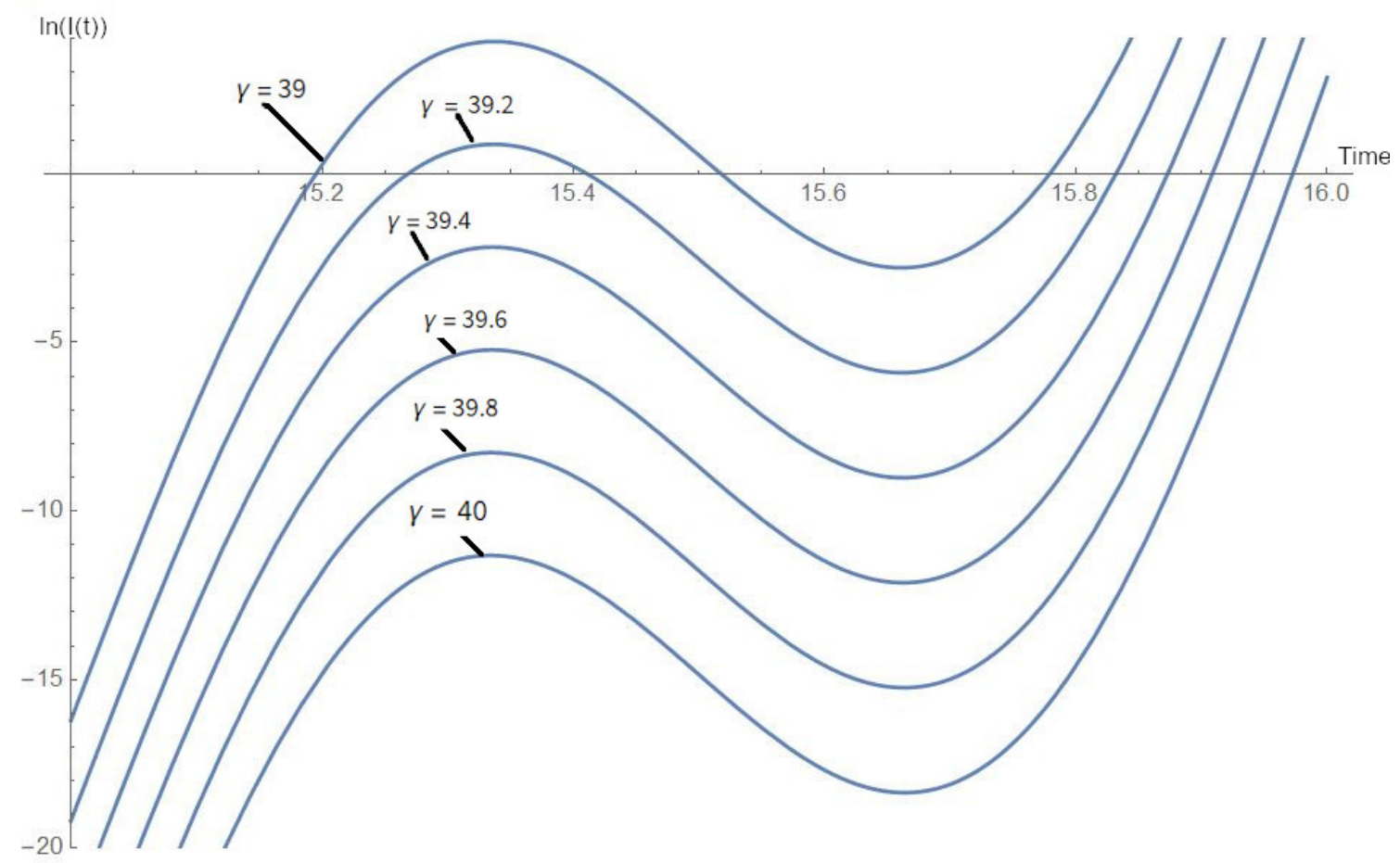

Figure 10: $\ln \tilde{I}_{\mathrm{II}}(t)$ plotted for varying values of $\gamma$. Note the limited interval about the integer values of $t$ where the initial root (corresponding to an outbreak) may be. While several of the curves intersect the horizontal axis more than once, only the first intersection may indicate an outbreak, as the intersection is the point where the approximations used must break down. Outbreaks near $t=15.85$ may occur because, as is visible in the $\gamma=39.4$ case, the initial local maximum may not be large enough to cause an outbreak, thus delaying the outbreak until the subsequent intersection. No outbreaks may occur in the interval from approximately $t=15.3$ to $t=15.85$.

Substituting the value for $b$ allows easier comparison:

$$
\frac{\beta_{0}}{\mu}-\frac{\mu \beta_{0} \delta}{4 \pi^{2}+\mu^{2}}(2 \pi+\mu)<\frac{\beta_{0}}{\mu}+\frac{\mu \beta_{0} \delta}{4 \pi^{2}+\mu^{2}}\left(\mu \cos \left(2 \pi t_{1}\right)-2 \pi \sin \left(2 \pi t_{1}\right)\right)<\frac{\beta_{0}}{\mu}+\frac{\mu \beta_{0} \delta}{4 \pi^{2}+\mu^{2}}(2 \pi+\mu) .
$$

To understand this better, we may consider the ranges of parameters that are of interest. The connection matching to the real-life quantities that the parameters represent allows us to make assumptions that are generally applicable to meaningful scenarios. First, note that, according to Pew Research [2], annual population growth $\mu$ is approximately 1\%, justifying the assumption that $\mu=O\left(10^{-2}\right)$. We may then compare the relative magnitudes of the remaining terms using $\mu$ as a base line. $\delta$ is confined to being between 0 and 1 for meaningful results, where the long inter-outbreak period solutions have been found for the upper range, so we may treat $\delta$ as $O\left(\mu^{1 / 2}\right)$. The values of $\gamma$ used typically range between 0 and 100, and so we may take $\gamma$ to be $O\left(\mu^{-1 / 2}\right)$. Lastly, we may treat $\beta_{0}$ as $O\left(\mu^{-1}\right)$, conservatively, and note that $4 \pi^{2}$ is at the same order as $\gamma$ roughly. Substituting these proportionality expressions in, we have that

$$
\frac{\beta_{0}}{\mu}=O\left(\frac{\mu^{-1}}{\mu}\right)=O\left(\mu^{-2}\right)
$$




\section{$\operatorname{Ln}(\mathrm{S}(\mathrm{t}) /(\mathrm{t}))$}

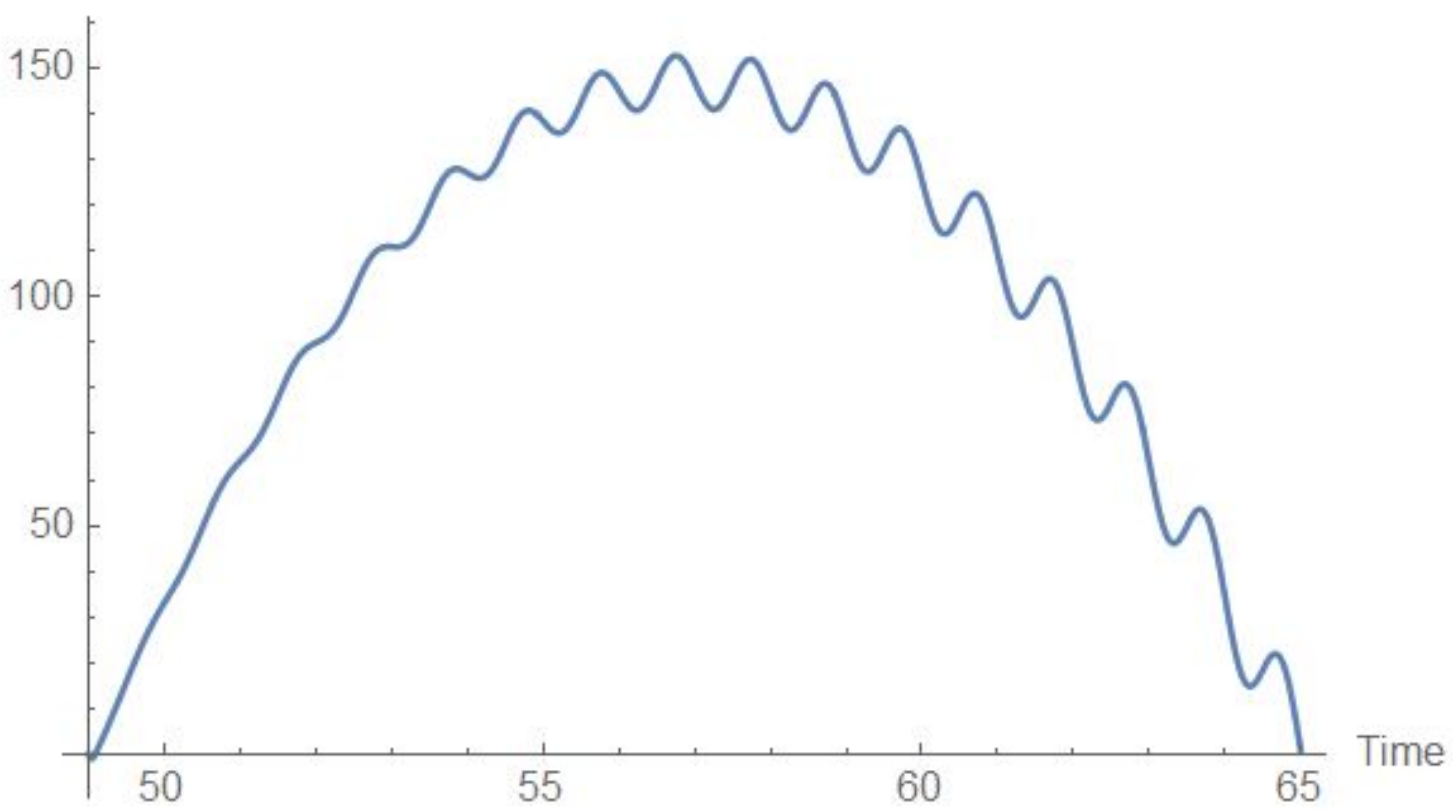

Figure 11: ln-scaled ratio of $\tilde{S}(t)$ and $\tilde{I}(t),\left(\beta_{0}, \delta, \gamma, \mu\right)=(500,0.9,40,0.01)$

$$
\frac{\mu \beta_{0} \delta}{4 \pi^{2}+\mu^{2}}(2 \pi+\mu)=O\left(\frac{\mu \mu^{-1} \mu^{1 / 2}}{\mu^{-1 / 2}}\left(\mu^{0}\right)\right)=O\left(\mu^{0}\right) .
$$

It is clear that the $\frac{\beta_{0}}{\mu}$ term will dominate. We can next see that

$$
\tilde{I}_{\mathrm{II}}(t) \approx I_{1} \exp \left(c+\phi t+\frac{\beta_{0} \delta \sin (2 \pi t)}{2 \pi}+\hat{S}(t)\left[\frac{\beta_{0}}{\mu}\right]\right) .
$$

While it is not possible to solve for $t_{C}$ such that $\lim _{t \rightarrow t_{C}} \frac{\tilde{I}_{\text {II }}(t)}{\tilde{S}_{\text {II }}(t)}=1$, it is possible to determine when $\tilde{I}_{\mathrm{II}}(t)$ leaves the realm of valid values. Particularly, no quantity in this system should ever exceed 1 , as the total population has been normalized. As such, we can attempt to solve for $t_{C}$ such that $\lim _{t \rightarrow t_{C}} \tilde{I}_{\mathrm{II}}(t)=1$ to find where the relationship between $I(t)$ and $S(t)$ will break down:

$$
\begin{gathered}
I_{1} \exp \left(c+\phi t_{C}+\left(S_{1}-1\right) e^{-\mu t_{C}}\left[\frac{\beta_{0}}{\mu}\right]\right)-1=0 \\
\Longrightarrow c+\phi t_{C}+\left(S_{1}-1\right) e^{-\mu t_{C}}\left[\frac{\beta_{0}}{\mu}\right]+\ln I_{1}=0 \\
\Longrightarrow t_{C}=\frac{\frac{\beta_{0}\left(1-S_{1}\right)}{\mu} e^{-\mu t_{C}}-c-\ln I_{1}}{\phi} .
\end{gathered}
$$

To solve this equation, we may call upon the Lambert $W$ function, discussed in Section 2.1 for what will be the first of several appearances. In our case, we can make the associations that

$$
\alpha \rightarrow \frac{-c-\ln I_{1}}{\phi}, \quad \nu \rightarrow \frac{\beta_{0}\left(1-S_{1}\right)}{\mu \phi}, \quad \chi \rightarrow-\mu
$$

We must also note that, as shown in Figure 12, the simplified form will cross the horizontal axis 


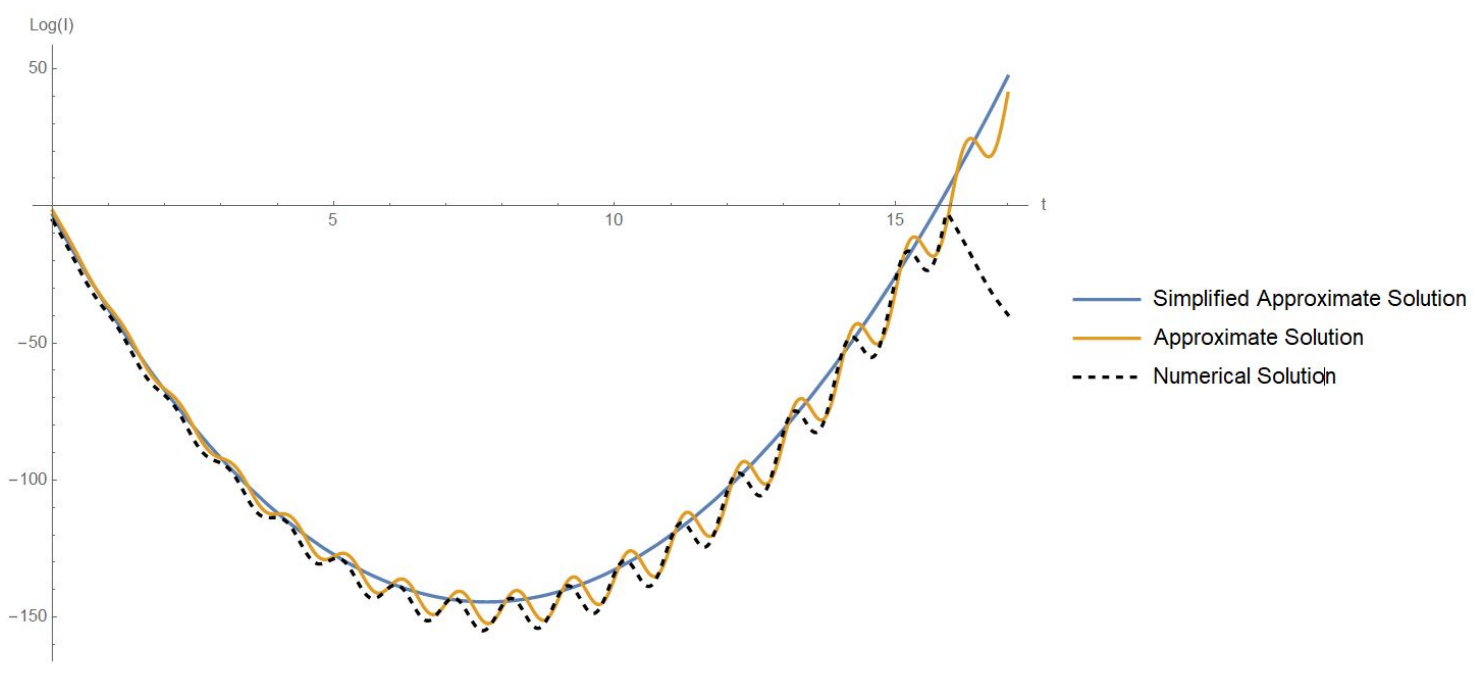

Figure 12: Comparing $\ln I(t)$ obtained by numerical solution vs. using the full approximate solution vs. using the simplified approximation from equation (28). Note that the simplified approximation crosses the $t$-axis early.

early, thus leading to an inaccurate $t_{C}$. The impact of this inaccuracy is reduced significantly upon applying requirement that outbreaks must occur at integer time values. Knowing this, we will round $t_{C}$ to the nearest integer.

Applying the associations from above, we now have

$$
t_{C}=\left\lfloor\frac{1}{\mu} W_{0}\left(\beta_{0} \phi^{-1}\left(1-S_{1}\right) e^{\frac{\mu\left(1+\ln I_{1}\right)}{\phi}}\right)-\frac{\left(c+\ln I_{1}\right)}{\phi}\right\rceil
$$

Where $\lfloor x\rceil$ is used to notate the nearest integer, and it is now specified that we are taking the principal branch of the Lambert $W$ function. It is worth noting that the $W_{-1}$ branch gives a meaningful solution as well, particularly indicating how far $t_{1}$ is from the other solution to $I(t)=1$.

We can now conclude that the dominant balance assumptions that have allowed the approximate solution will be valid in the region

$$
t_{1}+\epsilon<t<t_{1}+\frac{1}{\mu} W_{0}\left(\beta_{0} \phi^{-1}\left(1-S_{1}\right) e^{\frac{\mu\left(1+\ln I_{1}\right)}{\phi}}\right)-\frac{\left(c+\ln I_{1}\right)}{\phi}-\epsilon
$$

for some $\epsilon>0$. If it is defined that $t_{1}$ is the earliest possible time when the dominant balance assumptions are valid, then the $W_{-1}$ branch may be used to determine a lower bound on the end point for the region of validity. In other words, if we know that the dominant balance assumptions are valid after $t_{D}$ time units from the other solution to $I(t)=1$, then we may expect that our current solution will be valid up to $t_{D}$ time units before the next solution. 


\subsection{Outbreak Region}

Having shown that the assumptions made in the construction of the inter-outbreak solutions are valid and that with appropriate initial conditions, they may match numerical simulations with a great degree of accuracy, we may now turn our attention to finding a suitable approximation for the solution within the outbreak region.

We shall take a similar approach to that seen above, beginning the process by making reasonable assumptions.

\subsubsection{Simplifying Assumptions}

As discussed previously, this thesis is concerned with the behaviour of solutions to the S-SIR model where there is a long inter-outbreak period, followed by a short period of rapid outbreak.

That rapidity in the outbreak region is what allows a significant simplification in the region, to the point that a parametric solution is possible. Furthermore, the form of the solution will allow simplification to direct, closed-form expressions.

We will begin with a reminder of the equations at hand, first stated in Section 3.3:

$$
\begin{gathered}
S^{\prime}(t)=N \mu-\mu S(t)-\beta_{0}(1+\delta \cos 2 \pi t) I(t) S(t) \\
I^{\prime}(t)=\beta_{0}(1+\delta \cos 2 \pi t) I(t) S(t)-(\gamma+\mu) I(t),
\end{gathered}
$$

where we will take $N$ to be 1 .

The first assumption we may make is that relative to the period of the seasonal forcing, the outbreak occurs over a near-infinitesimal interval of time. This allows the reasonable assumption that the seasonal forcing term is near constant. In addition, we may see that based on prior numerical results that the outbreaks always occur at nearly integer values of $t$, thus we may impose the condition that $t \in \mathbb{Z}$, such that we may approximate $1+\delta \cos 2 \pi t$ as $1+\delta$. The phase of the forcing is chosen such that $t=0$ corresponds to a maximum of the forcing term.

Next, we note that because of the greatly varying scales of the parameters, $\mu$ is so small in comparison to $\gamma$ and $\beta_{0}$ that it may be treated as negligible, as may the $\mu S(t)$ term. Applying this, we arrive at

$$
\begin{aligned}
S^{\prime}(t) & =-\beta_{0}(1+\delta) I(t) S(t) \\
I^{\prime}(t) & =\beta_{0}(1+\delta) I(t) S(t)-\gamma I(t) .
\end{aligned}
$$

At this point, it is worth explicitly acknowledging that (30) is equivalent to the basic SIR model 
first explained in Section 3.1, with the generic $\beta$ value in the original statement taking on the constant value $\beta_{0}(1+\delta)$.

Before continuing forward, we'll want to confirm that this reduction to the traditional SIR model is actually valid. Plotted out in Figure 13 are the relevant comparisons of the numerical solutions to the two models for the typical period 16 mode examined so far. As shown in the figure, it is clear that for long period modes, the reduction to the basic $S I R$ model leads to a negligible change in the behaviour over the course of the outbreak.

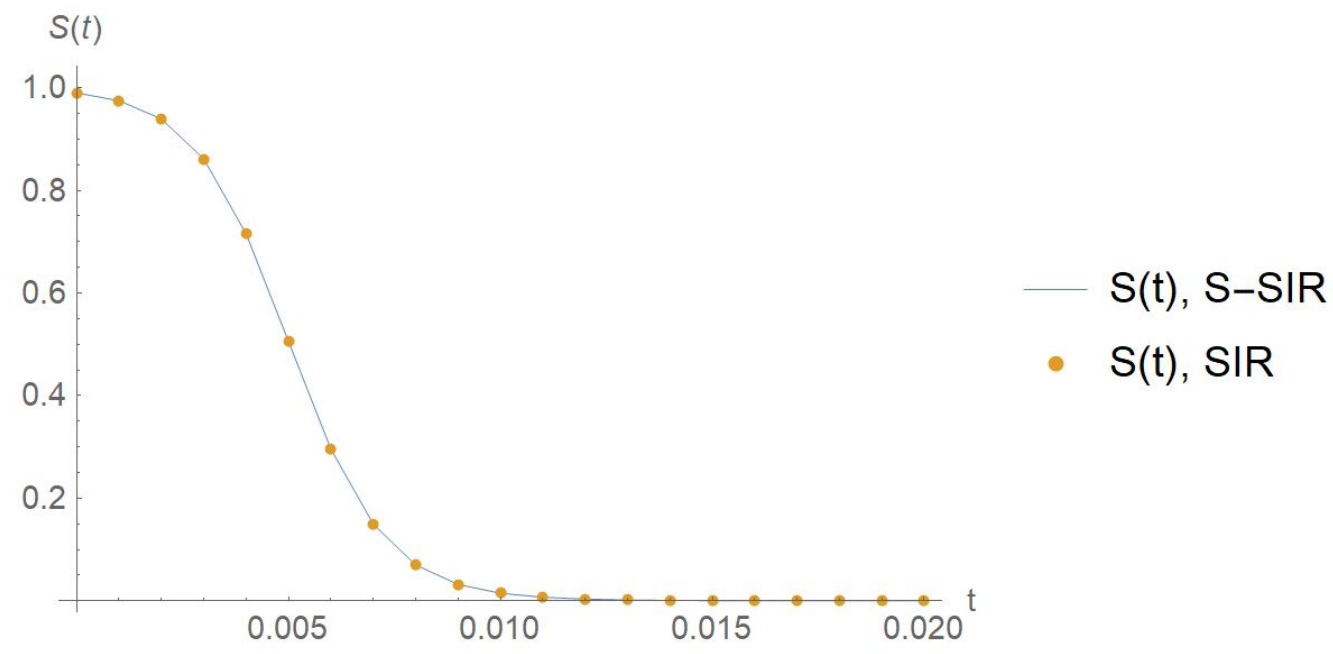

(a) Seasonal and basic outbreak numerical $\mathrm{S}(\mathrm{t})$

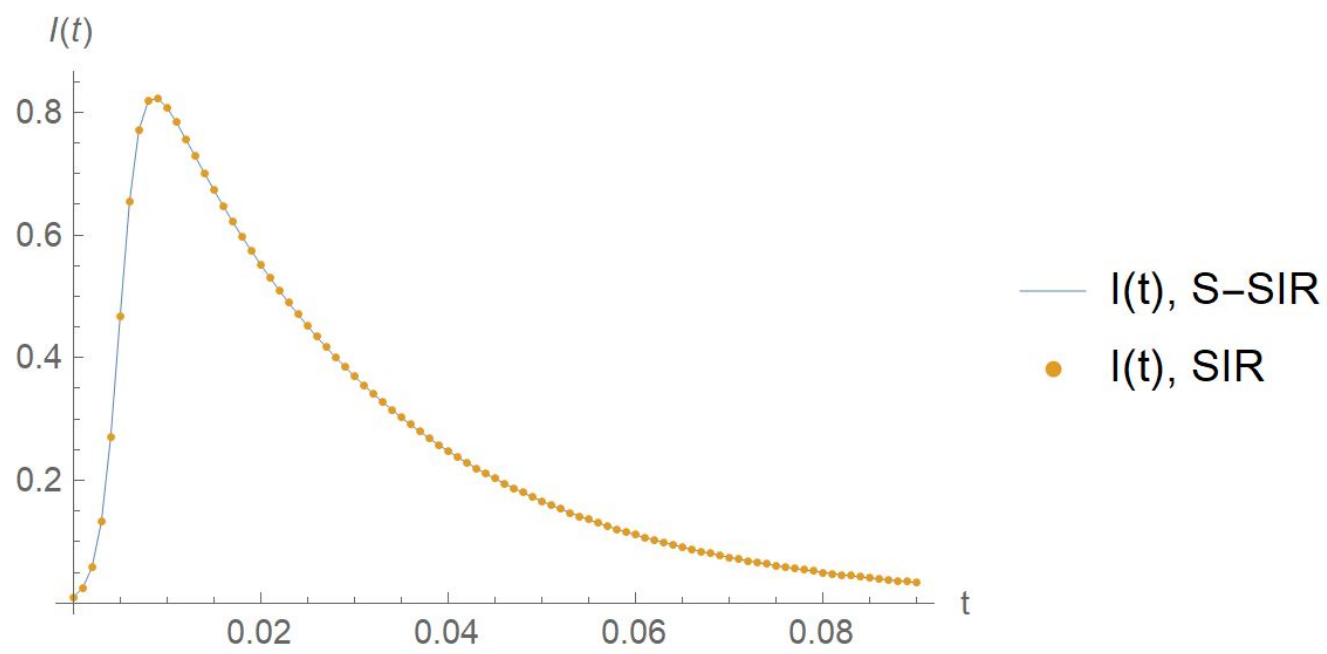

(b) Seasonal and basic outbreak numerical I(t)

Figure 13: Comparing the solution to the full seasonal SIR model and the traditional SIR model for $\left(\beta_{0}, \delta, \gamma, \mu\right)=(500,0.9,40,0.01)$. Note that at this time scale, the seasonality is not apparent. This is due to the extremely short time scale relative to the forcing. 


\subsubsection{Solving the SIR Model}

To begin the process of finding the parametric solution to the system shown, divide both sides of equation (30a) by $S(t)$ :

$$
\frac{S^{\prime}}{S}=-\beta_{0}(1+\delta) I(t)
$$

Next, note that

$$
\frac{d}{d t} \ln S(t)=-\beta_{0}(1+\delta) I(t)
$$

Inspired by that, we shall make the transformation $S(t)=e^{-G(t)}$, thus we have

$$
-\frac{d}{d t} G(t)=-\beta_{0}(1+\delta) I(t) .
$$

Now note that we have an expression for $I(t)$ in terms of $G(t)$

$$
I(t)=\frac{1}{\beta_{0}(1+\delta)}\left(\frac{d}{d t} G(t)\right)
$$

Note that from the compartment that has thus far seen the least use, we have

$$
\frac{d}{d t} R(t)=\gamma I(t)=\frac{\gamma}{\beta_{0}(1+\delta)}\left(\frac{d}{d t} G(t)\right),
$$

which can be integrated over time directly to yield

$$
R(t)=\frac{\gamma}{\beta_{0}(1+\delta)} G(t)+C .
$$

Now, applying the requirement of normalized and constant population, we have

$$
\begin{array}{r}
S+I+R=1 \\
e^{-G(t)}+\frac{G^{\prime}(t)}{\beta_{0}(1+\delta)}+\frac{\gamma}{\beta_{0}(1+\delta)} G(t)=1,
\end{array}
$$

which can then be solved for $G^{\prime}(t)$, yielding

$$
\frac{d G}{d t}=\beta_{0}(1+\delta)\left(1-e^{-G(t)}\right)-\gamma G(t) .
$$

We may then interchange the dependent and independent variables, arriving at

$$
\frac{d t}{d G}=\frac{1}{\beta_{0}(1+\delta)\left(1-e^{-G(t)}\right)-\gamma G(t)},
$$

so we may arrive at the conclusion that

$$
t(G)=\int_{a}^{G} \frac{d x}{\beta_{0}(1+\delta)\left(1-e^{-x}\right)-\gamma x},
$$

where the lower boundary, $a$, is subject to initial conditions. If we impose the condition that $S(t)$ begins near 1 , then we have

$$
S(0)=e^{-\iota}=e^{-G(0)} \Longrightarrow G(0)=\iota
$$


for some small $\iota$. Thus, $t(\iota)=0$, so we may let $a=\iota$. This now allows us to state an analytic solution to the traditional SIR model (which serves to approximate the solution to S-SIR model) in the outbreak region:

$$
\begin{aligned}
S(t) & =e^{-G(t)} \\
I(t) & =1-e^{-G(t)}-\frac{\gamma G(t)}{\beta_{0}(1+\delta)} \\
R(t) & =\frac{\gamma G(t)}{\beta_{0}(1+\delta)} \\
t(G) & =\int_{\iota}^{G} \frac{d x}{\beta_{0}(1+\delta)\left(1-e^{-x}\right)-\gamma x} .
\end{aligned}
$$

This result is technically a full solution, but is hindered by one significant problem: the integral in (36d) cannot be evaluated directly. To continue in a way that is less accurate but more meaningful, we'll need to find a street-fighting ${ }^{1}$ version of this solution. Before doing that, we'll need to verify that this formal solution is sufficient within the outbreak region.

\subsubsection{Verifying the Formal Solution}

In order to construct the parametric plots, we first must determine the range over which to vary

$G$. Examining (36d) we can see that the integrand approaches $\infty$ when the denominator vanishes; i.e., at $x$ such that $\beta_{0}(1+\delta)\left(1-e^{-x}\right)-\gamma x=0$. Labelling this value of $x$ as $G_{\infty}$, representing the limit of $G(t)$ as $t$ approaches $\infty$, we may rearrange to obtain

$$
G_{\infty}=\frac{\beta_{0}(1+\delta)}{\gamma}-\frac{\beta_{0}(1+\delta)}{\gamma} e^{-G_{\infty}}
$$

which has the familiar form seen in equation (4), which indicates then that the solution will be

$$
G_{\infty}=R_{0}+W_{0}\left(-R_{0} e^{-R_{0}}\right)
$$

where

$$
R_{0}=\frac{\beta_{0}(1+\delta)}{\gamma},
$$

where $R_{0}$ is recalled from Section 3.1, with the identification that $\beta \rightarrow \beta_{0}(1+\delta)$. Having $G_{\infty}$, it is now possible to plot out the parametric solution, varying $G$ between 0 and $G_{\infty}$. Figure 14 shows a comparison between the formal solution found here and a numerical solution of the full S-SIR model. Clearly, the formal solution found in equations (36) is more than sufficient for capturing the behaviour of the $S$-SIR model in the outbreak region.

\footnotetext{
${ }^{1}$ This is referring to a less exact, more practical version of the formal solution. Compare Tai-Chi to mixed-martial arts for an illustration. MIT offers an undergraduate course in approximate mathematics available by MIT Open Course Works, by the title "Street-Fighting Mathematics", and if they can use that terminology, so can I.
} 


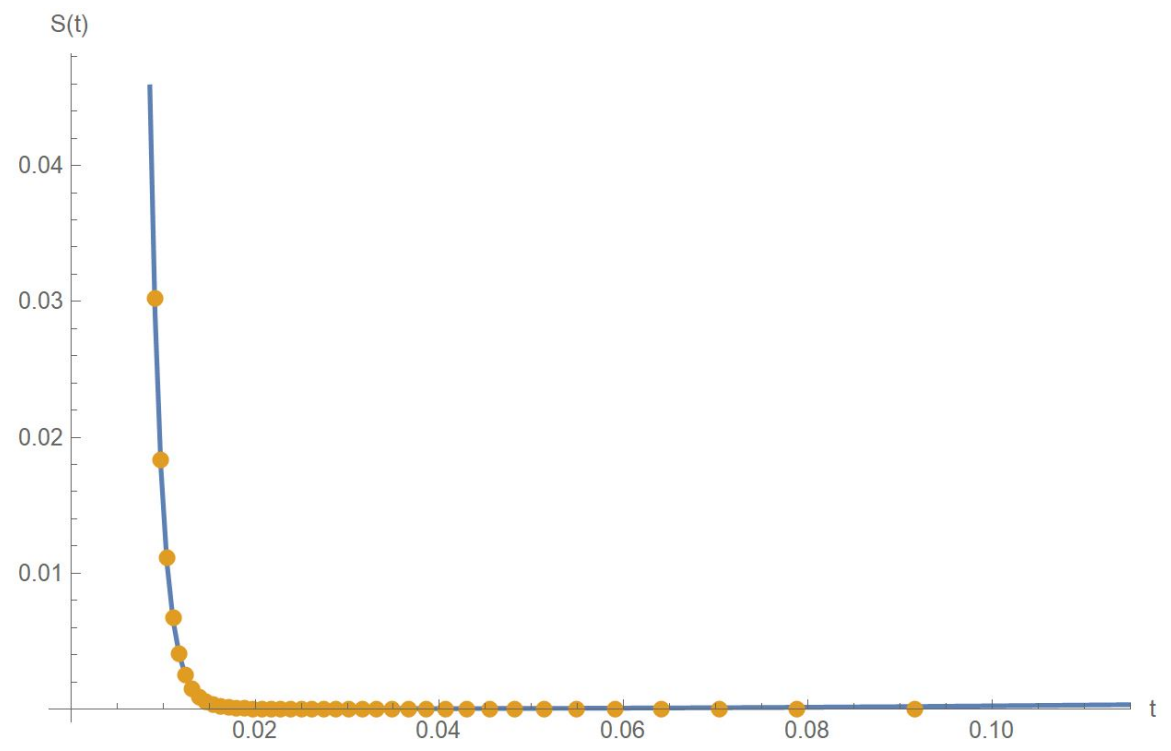

(a) $\mathrm{S}(\mathrm{t})$

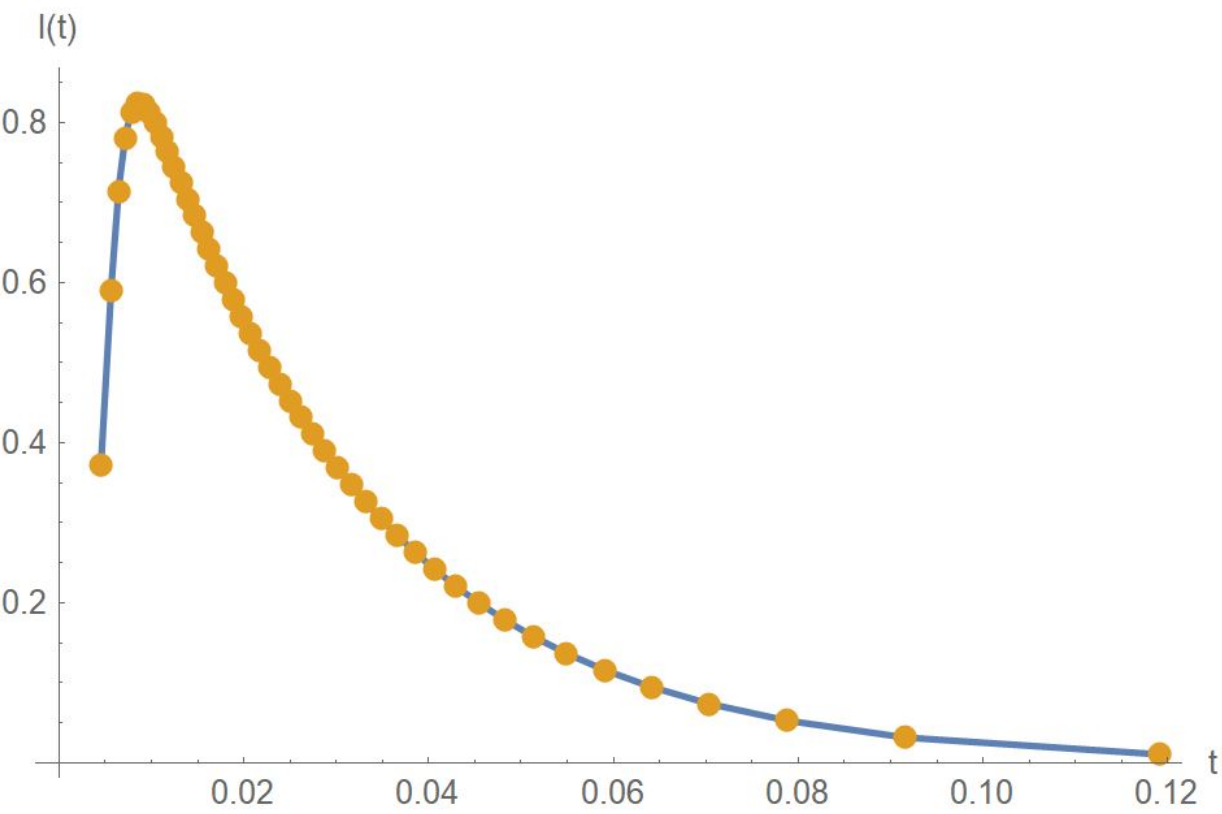

- Numerical Solution

- Formal Solution

(b) I(t)

Figure 14: Plotting discrete points of $\mathrm{S}(\mathrm{t})$ and $\mathrm{I}(\mathrm{t})$ from the formal solution (36) and numerical solution, $\left(\beta_{0}, \delta, \gamma, \mu\right)=(500,0.9,40,0.01)$ 


\section{Finding the Street-Fighting Outbreak Solution}

While we may be able to smugly claim we've solved the traditional $S I R$ model and technically not be wrong, the formal solution we've found is unwieldy and cannot be used to make many meaningful statements without numerical integration. If we're going to need to rely on the numerics, then we might as well solve the full $S$-SIR model numerically and call it a day. The goal we're seeking is something resembling a closed-form solution, even if it is an approximation.

To find our street-fighting solution will take a few intermediate steps. First, we'll construct a closed-form approximation of $t(G)$, then use that to find an approximation of $G(t)$. Having that, we'll have all we need to construct a closed-form approximate solution in the outbreak region.

This work is largely based on the procedure shown by Kroger and Schlickeiser [14], though an original effort for the justification and derivation of approximations used in said paper has been included.

\subsection{Finding the Peak Infection Rate, $\psi_{0}$}

Before proceeding to the direct construction of approximate functions, it will prove necessary to determine when the rate of infection during the outbreak will reach its maximum.

To begin, we can recognize that, based on equation (30a), the rate of infection (henceforth labelled $\psi(t))$ should simply be the negative of the derivative of $S$, yielding

$$
\psi(t)=\beta_{0}(1+\delta) S(t) I(t)
$$

Differentiation then indicates

$$
\psi^{\prime}(t)=\left(\beta_{0}(1+\delta)\right)^{2}\left(-S I^{2}+S^{2} I-\frac{S I \gamma}{\beta_{0}(1+\delta)}\right) .
$$

Dividing through by $\psi$ and recalling the definition of $R_{0}$ from Section 3.1 yields

$$
\frac{1}{\psi(t)} \psi^{\prime}(t)=\frac{d \ln \psi}{d t}=\beta_{0}(1+\delta)\left(S-I-R_{0}^{-1}\right) .
$$

This now indicates that $\ln \psi(t)$ (and therefore $\psi(t)$ ) will reach its maximum when $S-I=\frac{1}{R_{0}}$. Recalling equations (36) parts $a$ and $b$, we can express this in terms of $G$, indicating that the rate of infection will reach a maximum when

$$
2 e^{-G}-1+\frac{G}{R_{0}}=\frac{1}{R_{0}}
$$

This expression may now be rearranged into the form

$$
e^{-G}=\frac{R_{0}+1-G}{2 R_{0}} .
$$




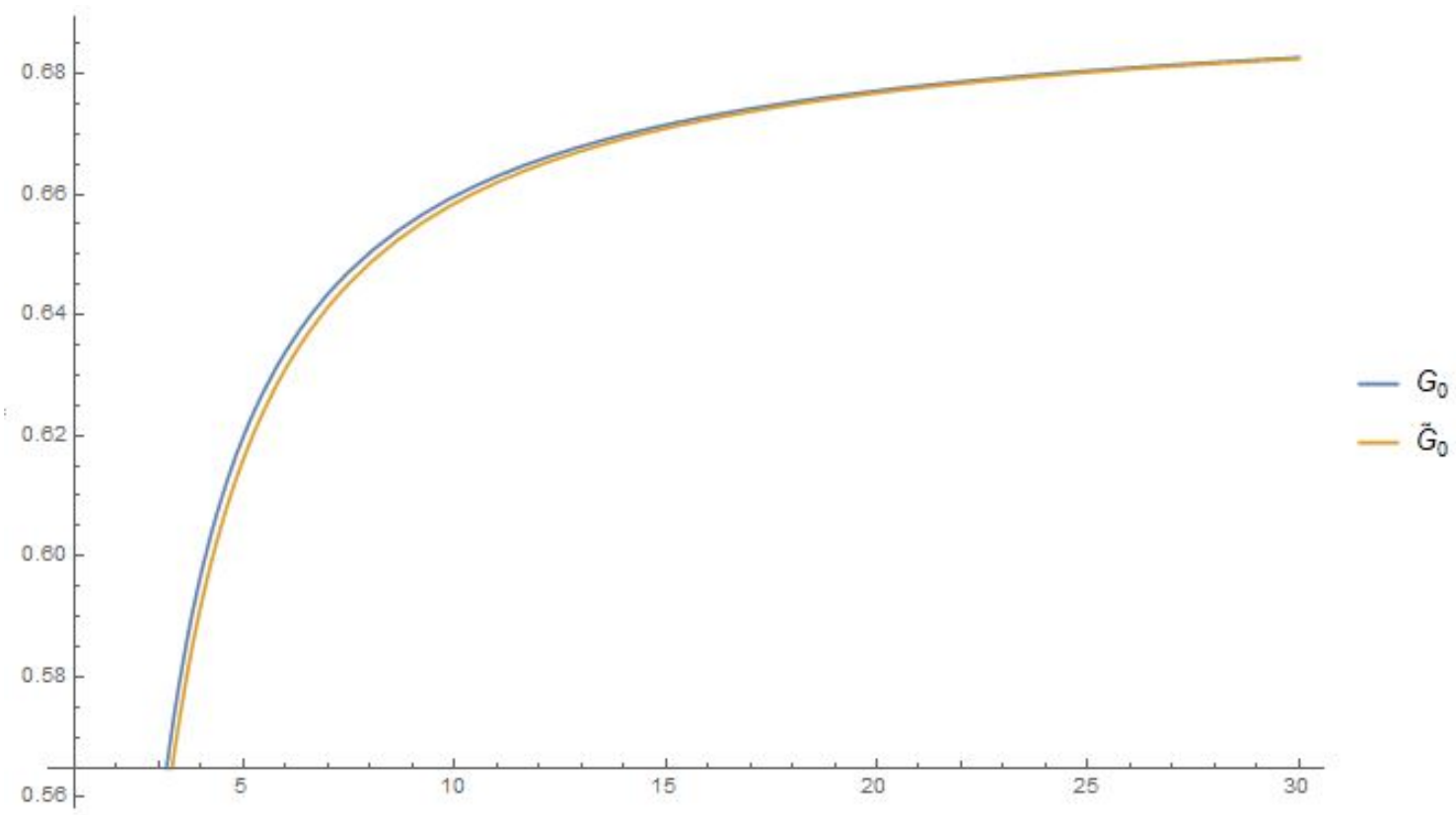

Figure 15: Comparing $G_{0}$ and the approximate $\tilde{G}_{0}$

which can be solved for $\mathrm{G}$ using the previously mentioned Lambert $W$ function, yielding

$$
G_{0}=1+R_{0}+W_{-1}\left(-2 R_{0} e^{-\left(R_{0}+1\right)}\right)
$$

where it must be noted that the non-principal root of the $W$ function is taken for consistency. If $W_{0}$ was used, then $G_{0}>G_{\infty}$, a clear contradiction.

In Kroger and Schlickeiser's work [14], the following approximation for $G_{0}$ was used

$$
G_{0} \sim\left(1-\frac{1}{R_{0}}\right)\left(1+\frac{5}{9 R_{0}}\right) \ln 2 \equiv \tilde{G}_{0},
$$

which yields a very accurate approximation for $R_{0}>1$, the range we are concerned with, as shown in Figure 15.

Substituting $G_{0}$ into equation (40), applying equation (44), then factoring and rearranging will yield the following:

$$
\begin{aligned}
\psi\left(G_{0}\right) & =\beta_{0}(1+\delta) e^{-G_{0}}\left(1-e^{-G_{0}}-\frac{G_{0}}{R_{0}}\right) \\
& =\beta_{0}(1+\delta)\left(1-\frac{R_{0}+1-G_{0}}{2 R_{0}}-G_{0}\right)\left(\frac{R_{0}+1-G_{0}}{2 R_{0}}\right) \\
& =\frac{\beta_{0}(1+\delta)\left(\left\{G_{0}-R_{0}\right\}^{2}-1\right)}{4 R_{0}^{2}} .
\end{aligned}
$$




\subsection{Approximating $t(G)$}

Recall equation (36d):

$$
t(G)=\int_{\iota}^{G} \frac{d x}{\beta_{0}(1+\delta)\left(1-e^{-x}\right)-\gamma x} .
$$

To determine an approximation for $t(G)$, we'll note a few of the key traits of the denominator of the integrand, which will be referred to as $f(x)$.

First, it will have a maximum at $x_{m}=\ln \frac{\beta_{0}(1+\delta)}{\gamma}$ and has two roots, $G_{\infty}$ and 0 . At this point, we know $f(0)=f\left(G_{\infty}\right)=0, f^{\prime}(0)=\beta_{0}(1+\delta)-\gamma, f^{\prime}\left(x_{m}\right)=0$, and

$$
f^{\max }=f\left(x_{m}\right)=\beta_{0}-\gamma+\beta_{0} \delta-\gamma \ln \frac{\beta_{0}(1+\delta)}{\gamma} .
$$

As a significant aside, it must be noted that, from equation (36), we have

$$
\frac{f(G(t))}{\beta_{0}(1+\delta)}=1-e^{-G(t)}-\frac{\gamma G(t)}{\beta_{0}(1+\delta)}=I(t),
$$

and thus $I(t)$ will reach its maximum at $G=x_{m}$ as well. Having this information, it is now possible to create asymptotic approximations of $f(x)$ in the limits of large and small $x$, by performing a Taylor expansion in the vicinity of 0 and of $G_{\infty}$. If

$$
f(x)=f(0)+f^{\prime}(0) x \quad \text { as } x \rightarrow 0,
$$

then we have that

$$
f(x) \sim\left(\beta_{0}(1+\delta)-\gamma\right) x \equiv h_{1}(x) \quad \text { as } x \rightarrow 0
$$

Similarly, we may expand about $G_{\infty}$, yielding

$$
f(x) \sim\left(e^{-G_{\infty}} \beta_{0}(1+\delta)-\gamma\right)\left(x-G_{\infty}\right) \equiv h_{2}(x) \quad \text { as } x \rightarrow G_{\infty} .
$$

We may now consider the relationship between $h_{1}(x)$ and $h_{2}(x)$ in both asymptotic limits.

It is clear to see from (53) and (52) that $h_{2}(x) \ll h_{1}(x)$ as $x \rightarrow G_{\infty}$, and similarly, $h_{1}(x) \ll h_{2}(x)$ as $x \rightarrow 0$. Recalling the definitions given in Section 2.2, we can observe that

$$
\frac{1}{g(x)} \ll \frac{1}{y(x)} \quad \text { as } x \rightarrow x_{0} .
$$

From this, we can then see that

$$
\begin{aligned}
& \frac{1}{h_{2}(x)} \ll \frac{1}{h_{1}(x)} \quad \text { as } x \rightarrow 0 \\
& \frac{1}{h_{1}(x)} \ll \frac{1}{h_{2}(x)} \quad \text { as } x \rightarrow G_{\infty} .
\end{aligned}
$$


Thus, we may relate the reciprocals of $f(x), h_{1}(x)$, and $h_{2}(x)$ as follows (see also Figure 17):

$$
\begin{aligned}
& \frac{1}{f(x)} \sim \frac{1}{h_{1}(x)} \sim \frac{1}{h_{1}(x)}+\frac{1}{h_{2}(x)} \quad \text { as } x \rightarrow 0 \\
& \frac{1}{f(x)} \sim \frac{1}{h_{2}(x)} \sim \frac{1}{h_{1}(x)}+\frac{1}{h_{2}(x)} \quad \text { as } x \rightarrow G_{\infty} .
\end{aligned}
$$

Thus, we have an asymptotic expansion for $f(x)^{-1}$ which is valid in both limits (see Figure 17).

$$
\frac{1}{f(x)} \sim \frac{1}{h_{1}(x)}+\frac{1}{h_{2}(x)} \quad \text { as } x \rightarrow 0 \text { and as } x \rightarrow G_{\infty} .
$$

Returning to the integral of interest, equation (36d), we may consider splitting apart the integral into three different portions, corresponding to "near $\iota "$, "near $G$ ", and "middle" regions.

$$
t(G)=\int_{\iota}^{g_{1}} \frac{1}{f(x)} d x+\int_{g_{1}}^{g_{2}} \frac{1}{f(x)} d x+\int_{g_{2}}^{G} \frac{1}{f(x)} d x
$$

Considering the first integral, we know first that $\iota$ is chosen to be a value very close to 0 , such that we may say

$$
\int_{\iota}^{g_{1}} \frac{1}{f(x)} d x \approx \int_{0}^{g_{1}} \frac{1}{f(x)} d x
$$

As is shown in texts on Asymptotic Methods [3], the asymptotic series near a given point of a function may be integrated term by term if the function is integrable about said point.

Thus,

$$
\int_{0}^{g_{1}} \frac{1}{f(x)} d x \sim \int_{\iota}^{g_{1}} \frac{1}{h_{1}(x)}+\frac{1}{h_{2}(x)} d x, \quad g_{1} \rightarrow \iota .
$$

Similarly, if we consider large values of $G$,

$$
\int_{g_{2}}^{G} \frac{1}{f(x)} d x \sim \int_{g_{2}}^{G} \frac{1}{h_{1}(x)}+\frac{1}{h_{2}(x)} d x, \quad g_{2} \rightarrow G .
$$

Figure 18 shows the accuracy of the approximation, particularly in the asymptotic behaviour which is of primary importance to us.

Lastly, we know that $f(x)$ will reach its maximum value in the region about $x=\ln \frac{\beta_{0}(1+\delta)}{\gamma}$; therefore $\frac{1}{f(x)}$ will reach its minimum in said region. Knowing this, we may solve for when both $\frac{1}{h_{1}(x)}$ and $\frac{1}{h_{2}(x)}$ must no longer be valid approximations of $\frac{1}{f(x)}$. Particularly, $g_{1}<x_{1}$, where $x_{1}$ is the solution of

$$
\frac{1}{\left(\beta_{0}(1+\delta)-\gamma-\gamma \ln \frac{\beta_{0}(1+\delta)}{\gamma}\right)}=\frac{1}{h_{1}\left(x_{1}\right)}
$$

which can be found to be

$$
x_{1}=\frac{\beta_{0}(1+\delta)-\gamma \ln \frac{\beta_{0}(1+\delta)-\gamma}{\gamma}}{\beta_{0}(1+\delta)-\gamma} .
$$




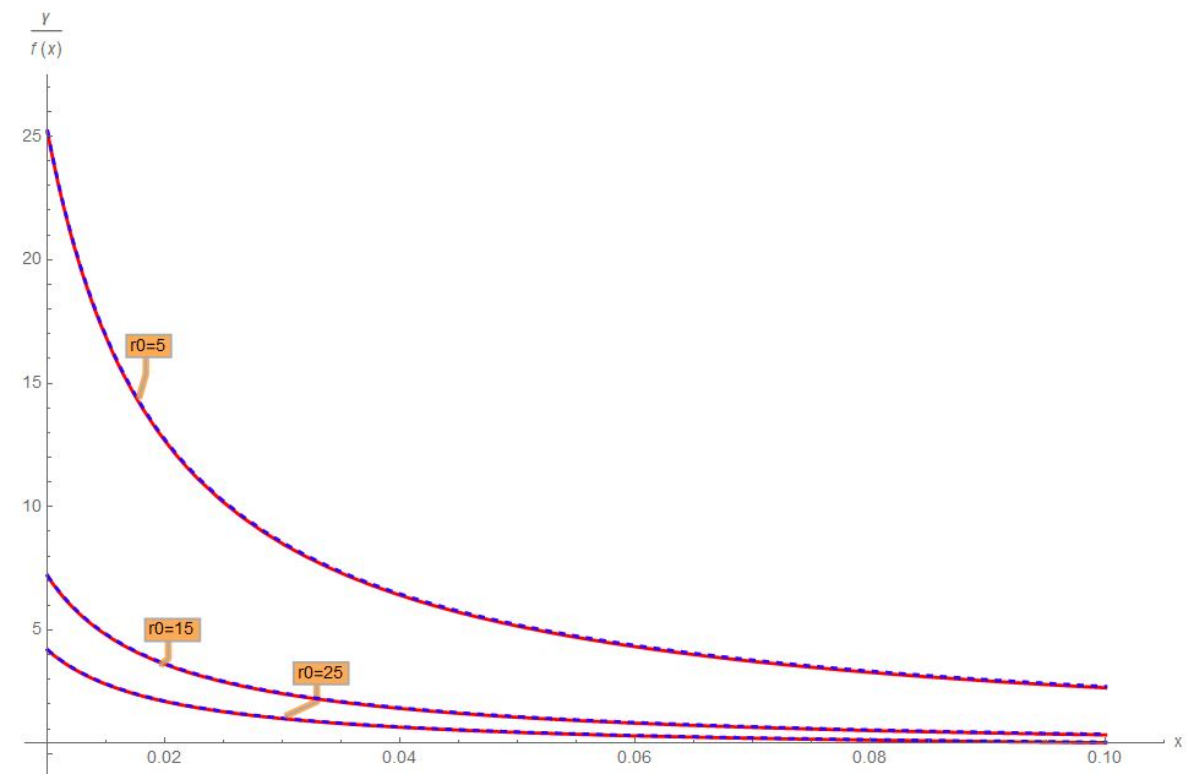

(a) $x \rightarrow 0$

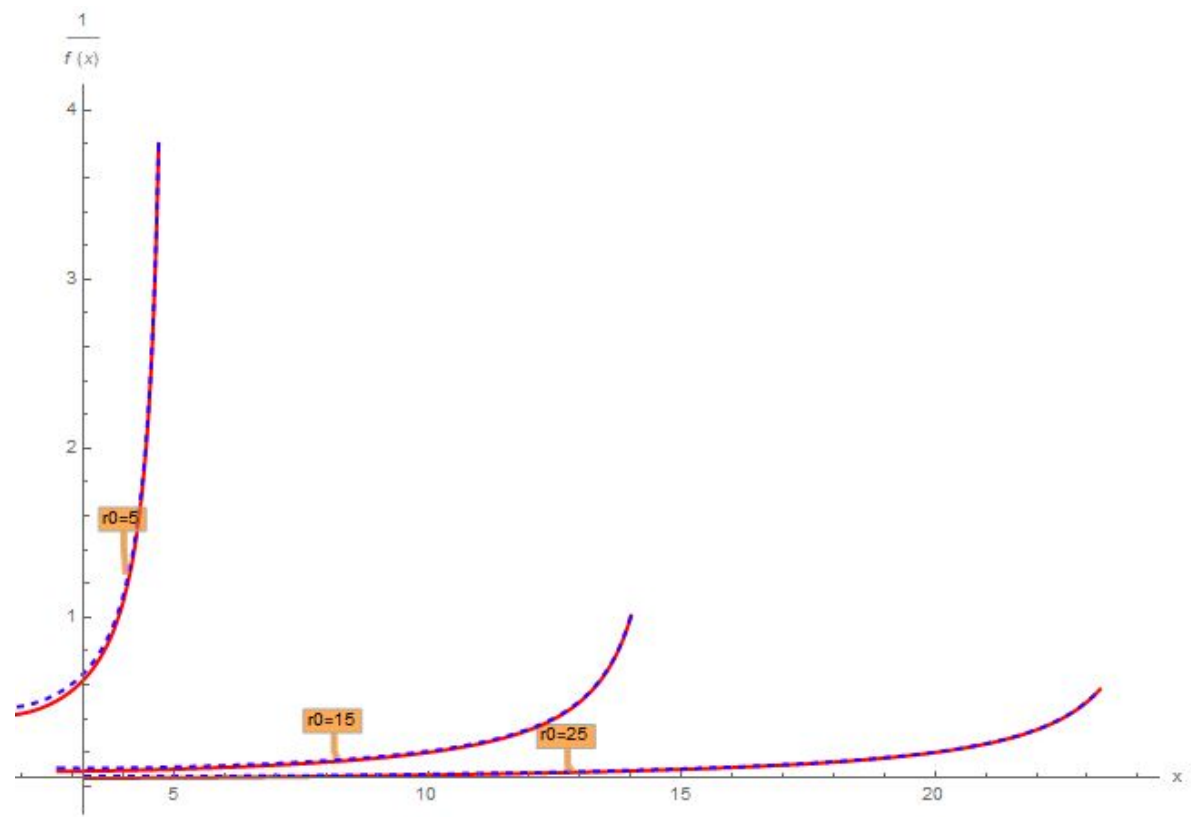

(b) $x \rightarrow G_{\infty}$

Figure 16: Comparing $\gamma / f(x)$ (solid) and $1 / h_{1}(x)+1 / h_{2}(x)$ (dashed) for varying $R_{0}$. Note that by multiplying through the $\gamma$ parameter, the graphs plotted are proportional only to the value of $R_{0}$ taken as a single parameter. 


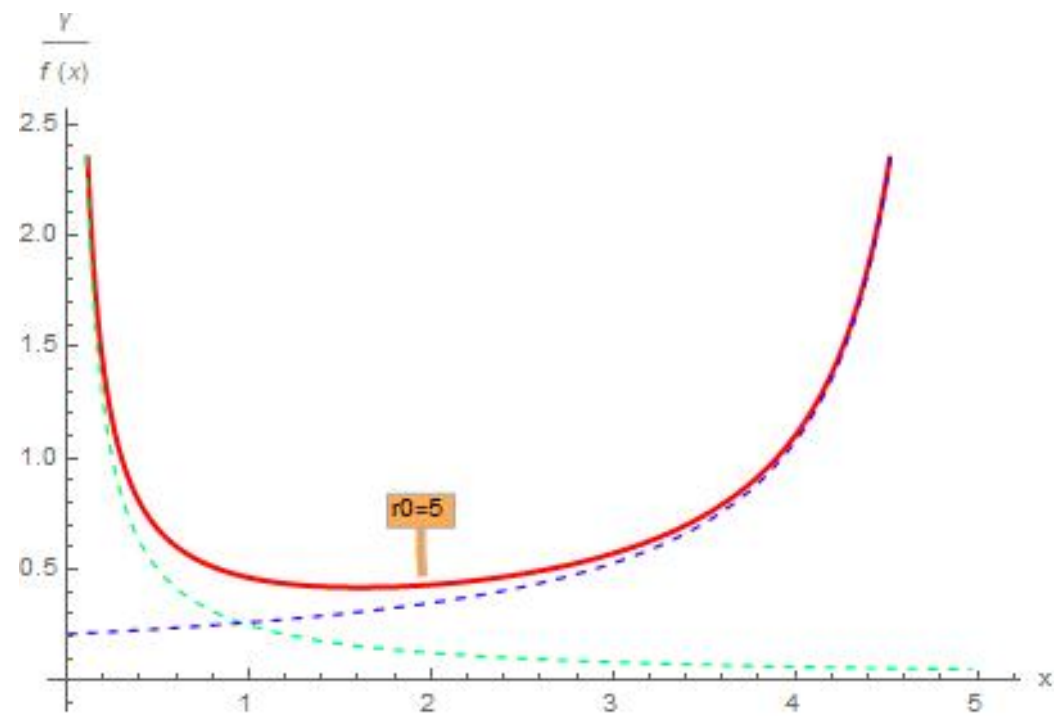

(a) $R_{0}=5$

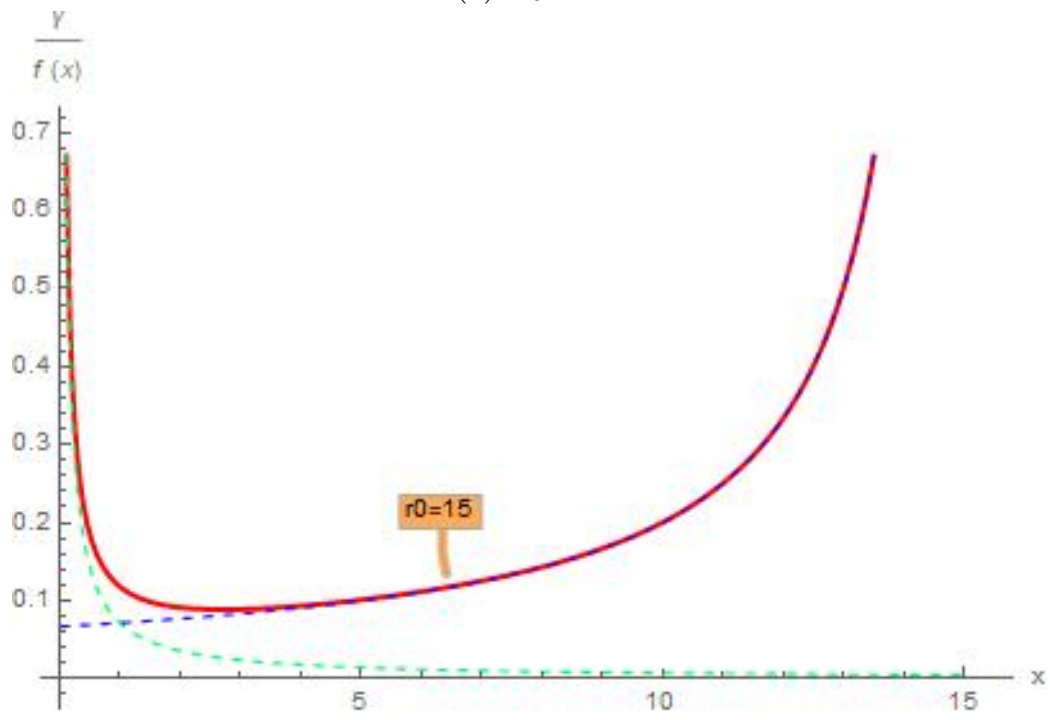

(b) $R_{0}=15$

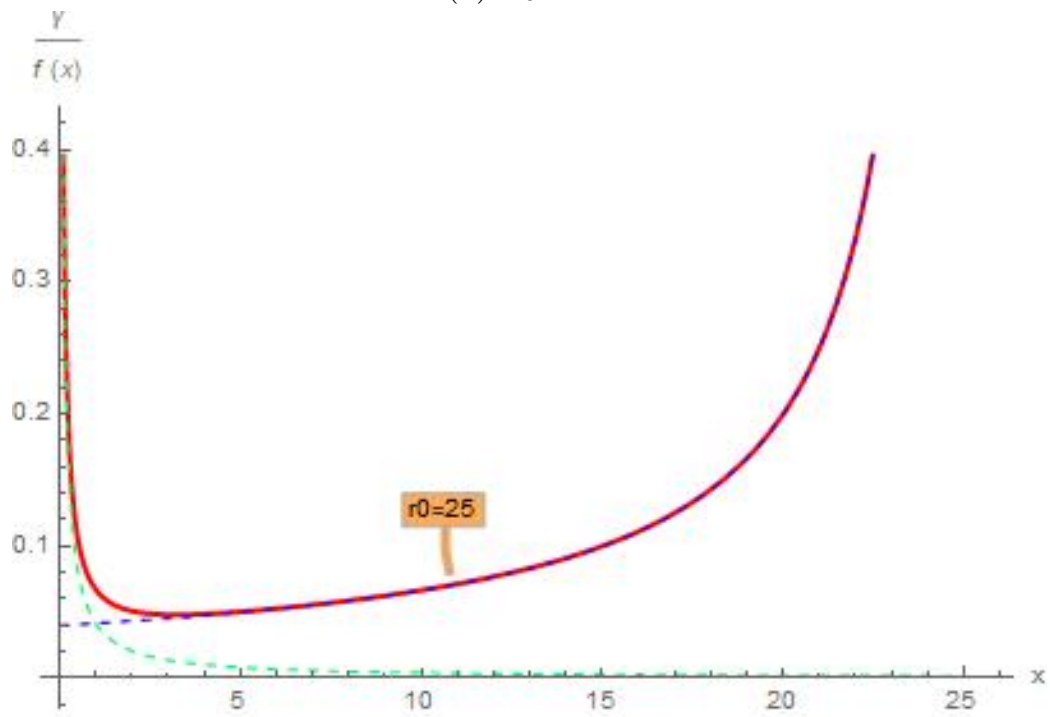

(c) $R_{0}=25$

Figure 17: Comparing $\gamma / f(x)$ (solid) $\gamma / h_{1}(x)$, and $\gamma / h_{2}(x)$ (dashed) for varying $R_{0}$, plotted over the entire domain 


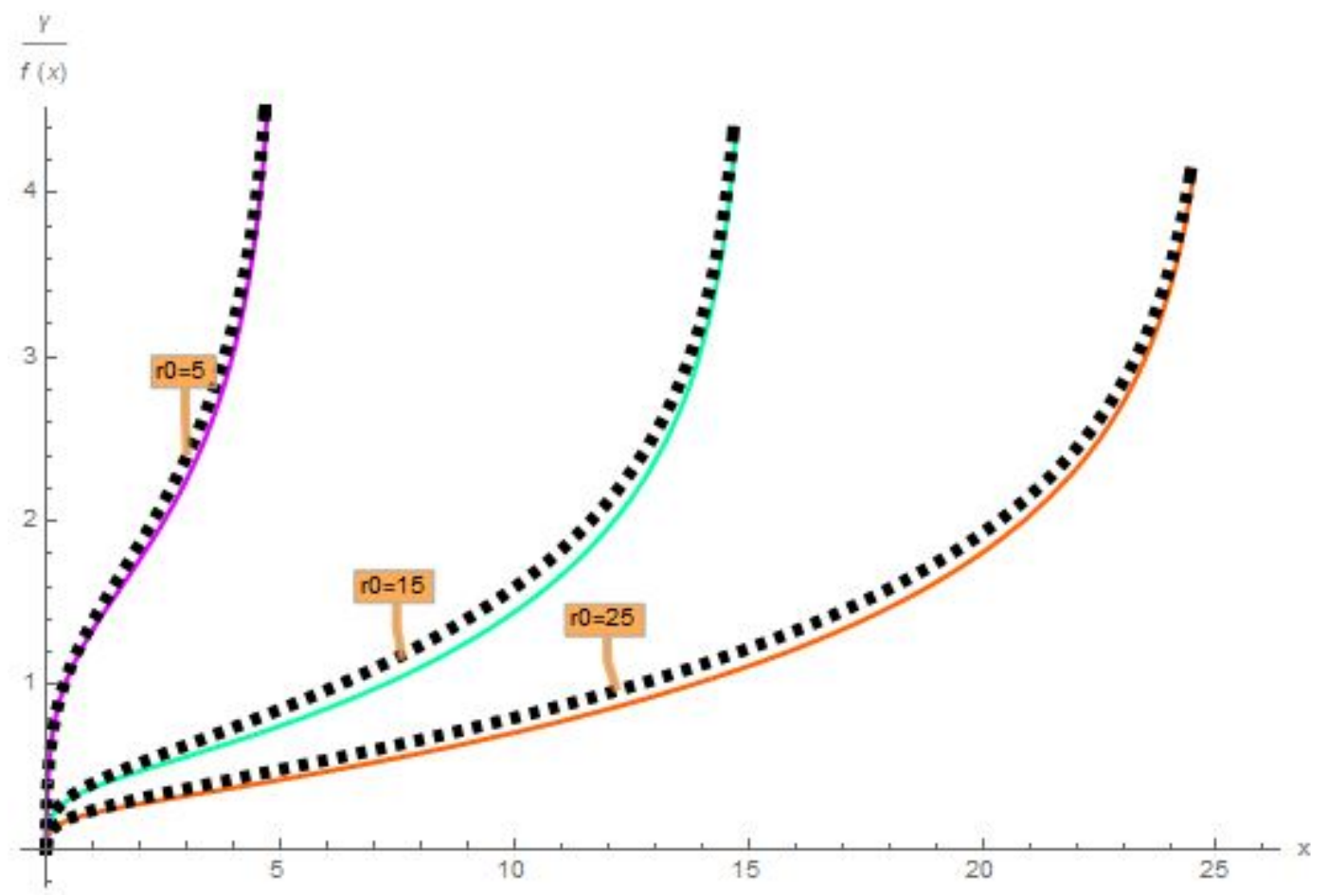

Figure 18: Comparison of $\gamma \int_{0.01}^{G_{\infty}} f(x)^{-1} d x$ (solid) and $\gamma \int_{0.01}^{G_{\infty}} h_{1}(x)^{-1}+h_{2}(x)^{-1} d x$ (dashed) for varying $R_{0}$

Similarly, defining $g_{2}>x_{2}$, it can be found that

$$
x_{2}=\frac{G_{\infty} \beta_{0}(1+\delta)+e^{G_{\infty}}\left(\beta_{0}(1+\delta)-G_{\infty}\right)-e^{G_{\infty}} \gamma \ln \frac{\beta_{0}(1+\delta)-\gamma}{\gamma}}{\beta_{0}(1+\delta)-\gamma} .
$$

We may then use the fact that

$$
\int_{a}^{b} \phi(x) d x \leq(b-a) \max _{a \leq x \leq b}(\phi(x)) .
$$

Thus, an approximation of the contribution of the "middle" integral in equation (57) can be found. Note that no claim of bounding is being made. Instead, $x_{1}$ and $x_{2}$ are the latest and earliest points for which the asymptotic approximations can be valid, respectively. Due to the fact that there is only one critical point of $\frac{1}{f(x)}$ in the interval $\left[x_{1}, x_{2}\right]$, which corresponds to a minimum, and $\frac{1}{f(x)}$ is continuous on the interval, we must find the maximum at either end of the interval. Thus,

$$
\int_{x_{1}}^{x_{2}} \frac{1}{f(x)} d x \leq\left(x_{2}-x_{1}\right) \max \left\{\frac{1}{f\left(x_{1}\right)}, \frac{1}{f\left(x_{2}\right)}\right\} .
$$

The preceding work, combined with the figures in this section, provides sufficient justification that the following is valid:

$$
t(G) \approx \int_{\iota}^{G} \frac{1}{f(x)} d x \sim \int_{\iota}^{G} \frac{1}{h_{1}(x)}+\frac{1}{h_{2}(x)} d x=\frac{\ln \left(\frac{G}{\iota}\right)}{\beta_{0}(1+\delta)-\gamma}+\frac{e^{G_{\infty}} \ln \frac{G-G_{\infty}}{\iota-G_{\infty}}}{\beta_{0}(1+\delta)-e^{G_{\infty} \gamma}} .
$$

We may confirm this approximation by taking the 2-norm of the distance between the functions,

$$
\left.d(f, g)\right|_{a} ^{b}=\sqrt{\frac{1}{b-a} \int_{a}^{b}\|f(x)-g(x)\|^{2} d x} .
$$




\begin{tabular}{l|l|l|l|l|l}
$R_{0}$ & $d(\gamma t(G), \gamma \tilde{t}(G))$ & $R_{0}$ & $d(\gamma t(G), \gamma \tilde{t}(G))$ & $R_{0}$ & $d(\gamma t(G), \gamma \tilde{t}(G))$ \\
\hline 2 & 0.0541055 & 13 & 0.0165075 & 24 & 0.00703483 \\
3 & 0.0510082 & 14 & 0.014956 & 25 & 0.00663362 \\
4 & 0.0469364 & 15 & 0.0136224 & 26 & 0.00626887 \\
5 & 0.042363 & 16 & 0.012469 & 27 & 0.00593614 \\
6 & 0.0377187 & 17 & 0.011465 & 28 & 0.00563167 \\
7 & 0.0333276 & 18 & 0.0105859 & 29 & 0.00535222 \\
8 & 0.0293748 & 19 & 0.00981143 & 30 & 0.00509502 \\
9 & 0.0259261 & 20 & 0.00912552 & 31 & 0.0048577 \\
10 & 0.0229699 & 21 & 0.00851487 & 32 & 0.00463816 \\
11 & 0.0204563 & 22 & 0.0079686 & 33 & 0.00443461 \\
12 & 0.0183227 & 23 & 0.00747774 & 34 & 0.00424548
\end{tabular}

Table 1: 2-norm of distance between $\gamma t$ and $\gamma \tilde{t}$ for varying $R_{0}$, using $\tilde{t}(G)$ as defined in equation (66c). Note the $\gamma$ scaling, allowing the functions in question to become dependent solely on the value of $R_{0}$

Having this, we may calculate the distance between $t(G)$ and the approximations in equation (64). These data are shown in Table 1.

Let

$$
\begin{aligned}
t_{1}(G) & =\frac{\ln \left(\frac{G}{L}\right)}{\beta_{0}(1+\delta)-\gamma} \\
t_{2}(G) & =\frac{e^{G_{\infty}} \ln \frac{G-G_{\infty}}{L-G_{\infty}}}{\beta_{0}(1+\delta)-e^{G_{\infty} \gamma}} \\
\tilde{t}(G) & =t_{1}(G)+t_{2}(G) .
\end{aligned}
$$

As suggested in equations (54) and (55), there is a regime in which $\frac{1}{h_{1}(x)}$ is dominant over $\frac{1}{h_{2}(x)}$, and one in which the opposite is true. To specify the regimes, we may solve for $x^{*}$ such that $h_{1}\left(x^{*}\right)=h_{2}\left(x^{*}\right)$, yielding

$$
x^{*}=\frac{\left(\beta_{0}(1+\delta)-\gamma e^{G_{\infty}}\right) G_{\infty}}{\beta_{0}(1+\delta)\left(1-e^{G_{\infty}}\right)} .
$$

Thus, we have that for $x<x^{*}, h_{1}(x)$ is dominant, while for $x>x^{*}, h_{2}(x)$ is dominant.

\subsection{Finding the Time of Peak Infection, $t_{p}$}

Having developed an approximation for $t(G)$, we may now use the fact that the rate of infection, $\psi(G)$, reaches a maximum at $G=G_{0}$, along with equation (66c) to find an approximation of when the infection will be spreading most rapidly. To begin, we introduce the abbreviations

$$
\begin{aligned}
\alpha & =-R_{0} e^{-R_{0}}, \\
\kappa & =\frac{W_{0}(\alpha)+1}{R_{0}-1}, \\
\text { and } \quad A(x) & =\ln \left(1-\frac{x}{G_{\infty}}\right)+\ln \left(\frac{G_{\infty}}{x}\right)^{\kappa} .
\end{aligned}
$$


We may then insert $G_{0}$ as found in equation (45) into equation (66c) and apply the above abbreviations to arrive at the expression

$$
t_{p} \equiv \tilde{t}\left(G_{0}\right)=\frac{R_{0}\left(A(\iota)-A\left(G_{0}\right)\right)}{\kappa\left(R_{0}-1\right)} .
$$

\subsection{Approximating $G(t)$}

Having found $\tilde{t}(G)$, we can begin the construction of $\tilde{G}(t)$, which will allow us to then create the approximations for $S$ and $I$. To begin, we may use equations (66a), (66b), and (66c), along with the arrangement found in (71) to arrive at the expression in terms of $G$,

$$
\tilde{t}=\frac{R_{0}(A(\iota)-A(G))}{\kappa\left(R_{0}-1\right)},
$$

which can then be rearranged to arrive at an implicit expression for $G$,

$$
-A(G)=\frac{t\left(R_{0}-1\right) \kappa}{R_{0}}-A(\iota) .
$$

Now, undoing the abbreviation for $A(G)$ term yields

$$
-A(G)=-\ln \left(1-\frac{G}{G_{\infty}}\right)-\ln \left[\left(\frac{G_{\infty}}{G}\right)^{\kappa}\right] .
$$

We then let

$$
g=\frac{G}{G_{\infty}}
$$

and

$$
g_{0}=\frac{G_{0}}{G_{\infty}} .
$$

Using equation (75a) in equation (74) gives

$$
-A(G)=-\ln (1-g)-\ln \left[\left(\frac{1}{g}\right)^{\kappa}\right],
$$

which may then be rearranged by application of the rules of logarithms to give

$$
-A(G)=\kappa \ln g-\ln (1-g) .
$$

Inserting back into equation (73) then results in the expression in terms of $g$,

$$
\kappa \ln g-\ln (1-g)=\frac{t\left(R_{0}-1\right) \kappa}{R_{0}}-A(\iota) .
$$

This may be rearranged to yield the transcendental equation

$$
\frac{g^{\kappa}}{1-g}=\exp \left(\frac{t\left(R_{0}-1\right) \kappa}{R_{0}}-A(\iota)\right),
$$




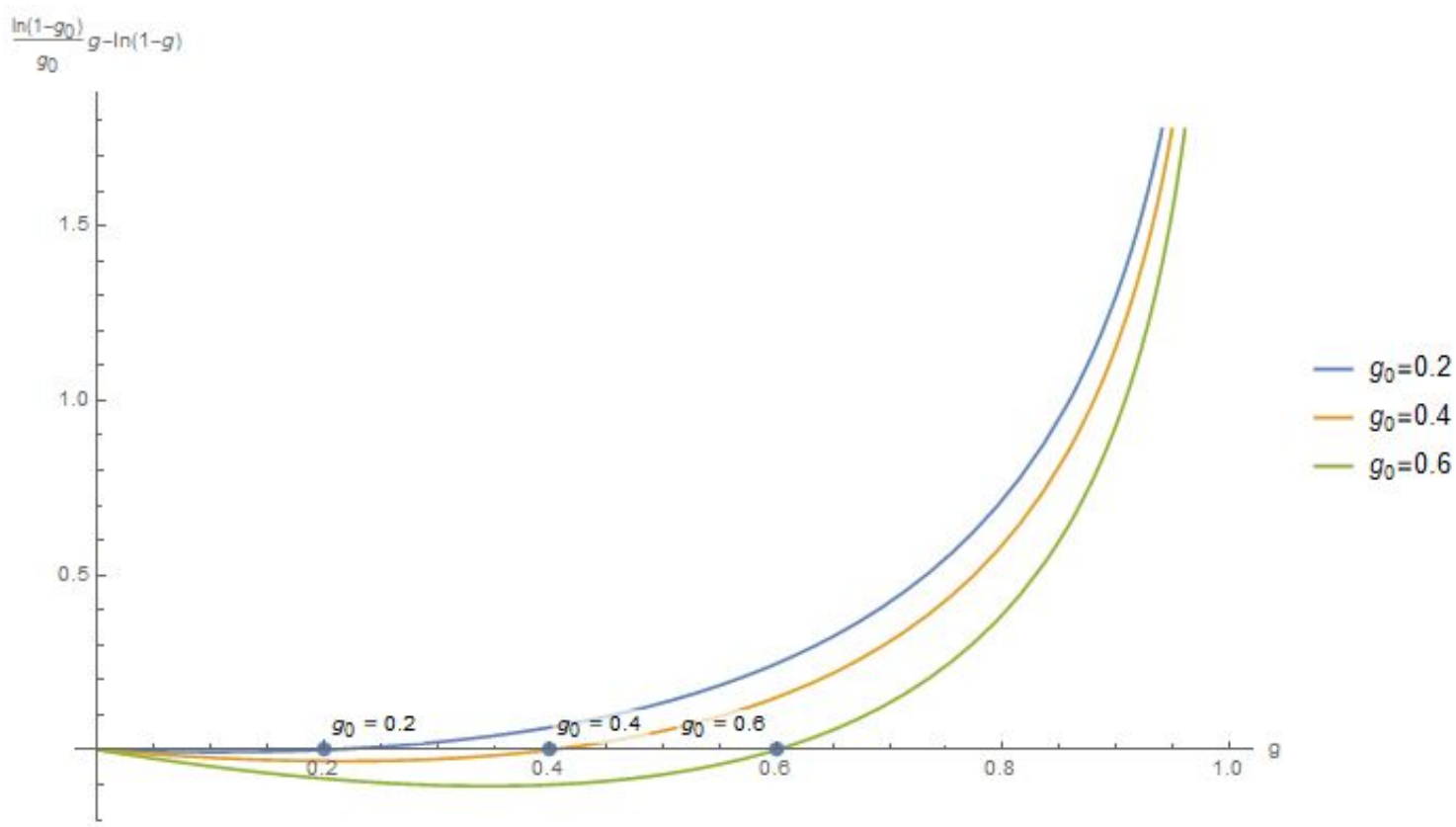

Figure 19: Plot of the difference between $\ln (1-g)$ and $\frac{\ln g\left(1-g_{0}\right)}{g_{0}}$. As can be seen, the approximation is exact at both $g=0$ and $g=g_{0}$ for $g_{0}=0.2,0.4,0.6$. For the purpose at hand, the primary requirement is the exact matching at the two roots, as shown in the figure. The increasing error as $g \rightarrow 1$ is of no concern, as this approximation will only be used for $0 \leq g \leq g_{0}$.

which, unfortunately, cannot be solved for $g$ directly. Instead, we shall approximate $g$ such that

$$
g= \begin{cases}g_{1}(t), & t \leq t_{p} \\ g_{2}(t) & t \geq t_{p}\end{cases}
$$

seeking a form such that $G$ and $\psi$ have the correct asymptotic behaviours and such that $g_{1}\left(t_{p}\right)=$ $g_{2}\left(t_{p}\right)$.

\subsubsection{Finding $g_{1}(t)$}

To approximate equation (76) in a way that is directly solvable, we must approximate $\ln (1-g)$ in such a way that it is exact at $g=0$ as well as at $g=g_{0}$. We can make the approximation

$$
\ln (1-g) \approx \frac{g \ln \left(1-g_{0}\right)}{g_{0}}
$$

As shown in Figure 19, this approximation has the necessary behaviour. If we then make the transformation $g=e^{-x}$, equation (76) becomes

$$
-\kappa x-e^{-x} \frac{\ln \left(1-g_{0}\right)}{g_{0}}=\frac{t\left(R_{0}-1\right) \kappa}{R_{0}}-A(\iota) .
$$

We may now rearrange the above equation into the form

$$
x=\left(\frac{A(\iota)}{\kappa}-\frac{t\left(R_{0}-1\right)}{R_{0}}\right)+\left(\frac{-\ln \left\{\left(1-g_{0}\right)\right\}}{g_{0} \kappa}\right) e^{-x},
$$


which is equivalent to equation ${ }^{2}(4)$, with $\xi=x, \alpha=\frac{A(\iota)}{\kappa}-\frac{t\left(R_{0}-1\right)}{R_{0}}, \nu=\frac{-\ln \left\{\left(1-g_{0}\right)\right\}}{g_{0} \kappa}$, and $\chi=-1$. Thus, we have the solution

$$
x=\left(\frac{A(\iota)}{\kappa}-\frac{t\left(R_{0}-1\right)}{R_{0}}\right)+W_{0}\left(\frac{-\ln \left\{\left(1-g_{0}\right)\right\}}{g_{0} \kappa} \exp \left\{\frac{t\left(R_{0}-1\right)}{R_{0}}-\frac{A(\iota)}{\kappa}\right\}\right) .
$$

For the time being, let us introduce the following abbreviations

$$
a_{1}=a_{1}(t)=\frac{A(\iota)}{\kappa}-\frac{t\left(R_{0}-1\right)}{R_{0}}
$$

and

$$
a_{2}=\frac{\ln \left\{\left(1-g_{0}\right)\right\}}{g_{0} \kappa},
$$

such that

$$
x=a_{1}(t)+W_{0}\left(a_{2} e^{-a_{1}(t)}\right) .
$$

Having this then implies that

$$
g_{1}=e^{-a_{1}} e^{-W_{0}\left(a_{2} e^{a_{1}}\right)} .
$$

Now, we may return to the definition of the Lambert $W$ from Section 2.1 to see that if $z=$ $W(z) e^{W(z)}$, then $e^{-W(z)}=W(z) / z$. Thus,

$$
e^{W_{0}\left(a_{2} e^{-a_{1}}\right)}=\frac{W_{0}\left(a_{2} e^{-a_{1}}\right)}{a_{2} e^{-a_{1}}},
$$

which then implies that equation (85) can be put into the form

$$
g_{1}(t)=\frac{W_{0}\left(a_{2} e^{-a_{1}(t)}\right)}{a_{2}} .
$$

\subsubsection{Finding $g_{2}(t)$}

For the other region, where $g$ is close to 1 , we may proceed in a way similar to the previous section.

The approximation we introduce must be exact at $g=1$ and $g=g_{0}$. Approximating

$$
\ln (g) \approx \frac{(1-g) \ln g_{0}}{1-g_{0}}
$$

has the desired behaviour, as shown in Figure 20. We proceed by substituting the approximation into equation (76), letting $g=e^{-x}$, and rearranging to arrive at the following form:

$$
x=\left(a_{1}-b_{1}\right)+b_{1} e^{-x},
$$

\footnotetext{
${ }^{2}$ Recall the use of the Lambert $\mathrm{W}$ function,

$$
\xi=\alpha+\nu e^{\chi \xi} \Longrightarrow \xi=\alpha-\frac{1}{\chi} W\left(-\nu \chi e^{\alpha \chi}\right) .
$$
}




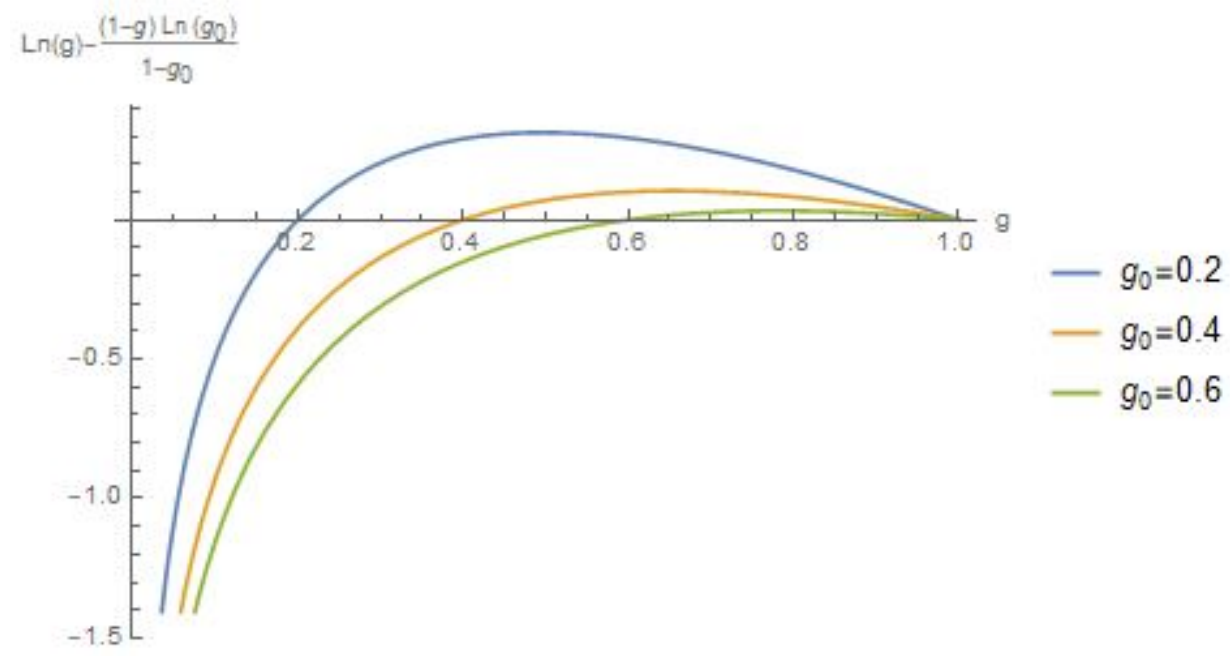

Figure 20: Plotting the difference between $\ln g$ and the approximation in equation (88). The goal is for the approximation to be exact at $g=g_{0}$ and and at $g=1$, which is shown to be true. The error as $g \rightarrow 0$ is of no concern, as the approximation will be used only in the region $g_{0} \leq g \leq 1$.

where $b_{1}=\frac{\ln g_{0}}{\kappa\left(1-g_{0}\right)}$. This yields the solution

$$
x=\left(a_{1}-b_{1}\right)+W_{0}\left(b_{1} e^{-\left(a_{1}-b_{1}\right)}\right),
$$

implying that

$$
g_{2}(t)=\frac{W_{0}\left(b_{1} e^{b_{1}-a_{1}(t)}\right)}{b_{1}}
$$

through a similar process as in the preceding section.

\subsubsection{Statement of $\tilde{G}(t)$}

Now that $g_{1}$ and $g_{2}$ have been derived, it is possible to state the approximation for $G(t)$.

$$
\tilde{G}(t)= \begin{cases}\frac{G_{\infty} W_{0}\left(a_{2} e^{-a_{1}(t)}\right)}{a_{2}} & t \leq t_{p} \\ \frac{G_{\infty} W_{0}\left(b_{1} e^{b_{1}-a_{1}(t)}\right)}{b_{1}} & t \geq t_{p}\end{cases}
$$


which uses the following abbreviations, reprinted here for ease of reference:

$$
\begin{aligned}
a_{1}(t) & =\frac{A(\iota)}{\kappa}-\frac{t\left(R_{0}-1\right)}{R_{0}} \\
a_{2} & =\frac{\ln \left\{\left(1-g_{0}\right)\right\}}{g_{0} \kappa} \\
b_{1} & =\frac{\ln g_{0}}{\kappa\left(1-g_{0}\right)} \\
\alpha & =-R_{0} e^{-R_{0}} \\
\kappa & =\frac{W_{0}(\alpha)+1}{R_{0}-1} \\
A(x) & =\ln \left(1-\frac{x}{G_{\infty}}\right)+\ln \left(\frac{G_{\infty}}{x}\right)^{\kappa} \\
R_{0} & =\frac{\beta_{0}(1+\delta)}{\gamma} \\
t_{p} & =\frac{R_{0}\left(A(\iota)-A\left(G_{0}\right)\right.}{\kappa\left(R_{0}-1\right)} \\
G_{\infty} & =R_{0}+W_{0}\left(-R_{0} e^{-R_{0}}\right) \\
G_{0} & =1+R_{0}+W_{-1}\left(-2 R_{0} e^{-\left(R_{0}+1\right)}\right) \\
g(t) & =\frac{G(t)}{G_{\infty}} \\
g_{0} & =\frac{G_{0}}{G_{\infty}}
\end{aligned}
$$

\subsection{The Street-Fighting Outbreak Solution, $\tilde{E}_{I}(t)$}

Having found $\tilde{G}(t)$, it is now possible to substitute equation (92) in equation (36) to state the approximate solution for the $S$-SIR model in the outbreak region:

$$
\begin{aligned}
& \tilde{E}_{I}(t)=\left(\begin{array}{c}
\tilde{S}_{I}(t) \\
\tilde{I}_{I}(t)
\end{array}\right) \\
& \tilde{S}_{I}(t)= \begin{cases}\exp \left(-\frac{G_{\infty} W_{0}\left(a_{2} e^{-a_{1}(t)}\right)}{a_{2}}\right) & t \leq t_{p} \\
\exp \left(-\frac{G_{\infty} W_{0}\left(b_{1} e^{b_{1}-a_{1}(t)}\right)}{b_{1}}\right) & t \geq t_{p}\end{cases} \\
& \tilde{I}_{I}(t)= \begin{cases}1-\exp \left(-\frac{G_{\infty} W_{0}\left(a_{2} e^{-a_{1}(t)}\right)}{a_{2}}\right)-\frac{G_{\infty} W_{0}\left(a_{2} e^{-a_{1}(t)}\right)}{k a_{2}} & t \leq t_{p} \\
1-\exp \left(-\frac{G_{\infty} W_{0}\left(b_{1} e^{b_{1}-a_{1}(t)}\right)}{b_{1}}\right)-\frac{G_{\infty} W_{0}\left(b_{1} e^{b_{1}-a_{1}(t)}\right)}{k b_{1}} & t \geq t_{p} .\end{cases}
\end{aligned}
$$

From Figure 21, this "Street-Fighting Solution" is highly accurate for the outbreak region. 


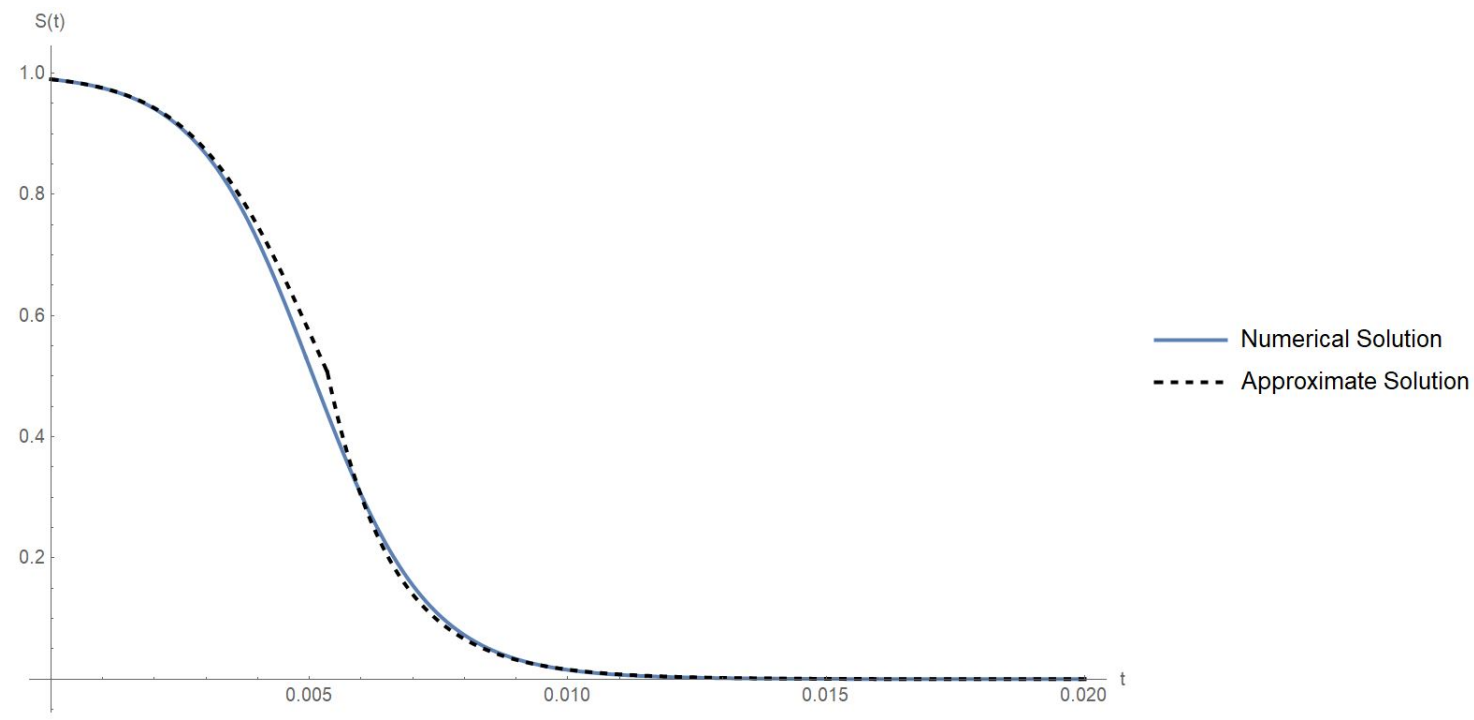

(a) $S(t)$

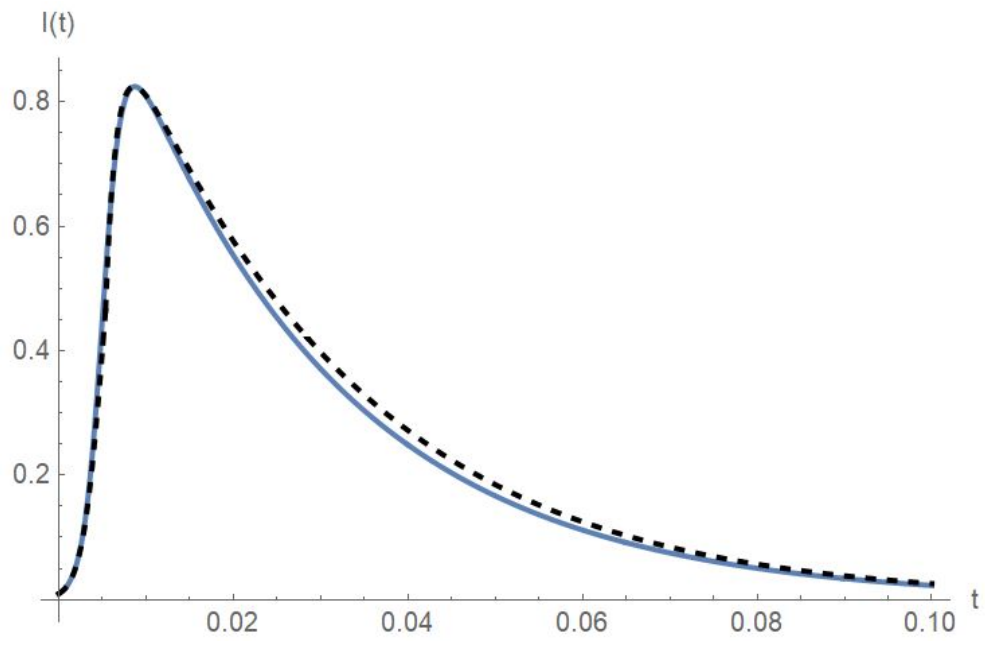

(b) $I(t)$

Figure 21: Comparing the numerical solution to $\tilde{E}_{I}(t)$ in the outbreak region $\left(\beta_{0}, \delta, \gamma, \mu\right)=$ $(500,0.9,40,0.01)$

\subsection{Finding a Uniformly Valid $\tilde{S}$}

Having found $\tilde{G}$, we now have an approximation for $\tilde{S}$ in the outbreak region, which will allow us to capture the behaviour during the outbreak.

This approximation has a significant issue, however. We can see that

$$
\begin{aligned}
& \lim _{t \rightarrow-\infty} \tilde{S}(t)=1, \\
& \lim _{t \rightarrow \infty} \tilde{S}(t)=S_{\infty} .
\end{aligned}
$$

These limits are entirely inconsistent with the behaviour in the inter-outbreak region. We will attempt to construct an approximation that is uniformly valid on the interval $t_{0}^{n}<t<t_{0}^{n+1}$ (i.e, from the beginning of the current outbreak, up until the beginning of the next outbreak). 
In pursuit of this, let us take a modified form of $\tilde{S}$,

$$
\tilde{S}_{U}^{n}(t)=e^{-\tilde{G}(t)}+1+c e^{-\mu t} .
$$

Now, let us consider the limiting behaviour at either side of $t_{1}^{n}$. We may note that, by definition and the necessary continuity of $S$, we have

$$
\lim _{t \rightarrow\left(t_{1}^{n}\right)^{-}} \tilde{S}_{I I}^{n-1}(t)=S_{\infty}+1+c e^{-\mu t_{1}}=S_{1}^{n} .
$$

Thus, we have

$$
c=\left(S_{1}^{n}-1-S_{\infty}\right) e^{-\mu t_{1}},
$$

yielding

$$
\tilde{S}_{I}^{n}(t)=e^{-G(t)}+1+\left(S_{1}^{n}-1-S_{\infty}\right) e^{-\mu\left(t-t_{1}\right)} .
$$

Using this result, we can see that

$$
\begin{aligned}
\lim _{t \rightarrow \infty} \frac{e^{-G(t)}+1+\left(S_{1}^{n}-1-S_{\infty}\right) e^{-\mu\left(t-t_{1}^{n}\right)}}{1+\left(S_{1}^{n-1}-1\right) e^{-\mu\left(t-t_{1}^{n-1}\right)}}=1 \\
\Longrightarrow \tilde{S}_{I}^{n}(t)+1+\left(S_{1}^{n}-1-S_{\infty}\right) e^{-\mu\left(t-t_{1}^{n}\right)} \sim \tilde{S}_{I I}^{n}(t), \quad t \rightarrow \infty,
\end{aligned}
$$

and

$$
\begin{aligned}
& \lim _{t \rightarrow-\infty} \frac{e^{-G(t)}+1+\left(S_{1}^{n}-1-S_{\infty}\right) e^{-\mu\left(t-t_{1}^{n}\right)}}{1+\left(S_{1}^{n-1}-1\right) e^{-\mu\left(t-t_{1}^{n-1}\right)}}=1 \\
& \quad \Longrightarrow \tilde{S}_{I}^{n}(t)+1+\left(S_{1}^{n}-1-S_{\infty}\right) e^{-\mu\left(t-t_{1}^{n}\right)} \sim \tilde{S}_{I I}^{n-1}(t), \quad t \rightarrow-\infty .
\end{aligned}
$$

Thus we now have a form that can be used to approximate $S(t)$ within both the outbreak and inter-outbreak regions. We shall define it as

$$
\tilde{S}_{U}^{n}(t)=e^{-G(t)}+1+\left(S_{1}^{n}-1-S_{\infty}\right) e^{-\mu\left(t-t_{1}^{n}\right)} .
$$

It must be noted that, due to the single degree of freedom introduced, exact matching on both sides of the outbreak region is not always possible. Applying the same process but matching at $t_{0}^{n}$ yields a similar form, which is more accurate in the region from $t_{1}^{n-1}$ to $t_{0}^{n}$ while being less accurate in the region from $t_{1}^{n}$ to $t_{0}^{n+1}$, but said "retroactive" form is not useful for the approach we will take.

Additionally, there is what may be considered as a problem of causality and knowledge. The stated form of $\tilde{S}_{U}^{n}(t)$ is dependent on $S_{1}^{n}$, which is not known a priori. There are a few ways of addressing this paradox, each sacrificing either the closed-form state or accuracy. Based on the 
results discussed in this section, we may note that if $S_{1}^{n}=S_{\infty}$, then we arrive at

$$
\tilde{S}_{U}^{n}(t)=e^{-G(t)}+1-e^{-\mu\left(t-t_{1}^{n}\right)}
$$

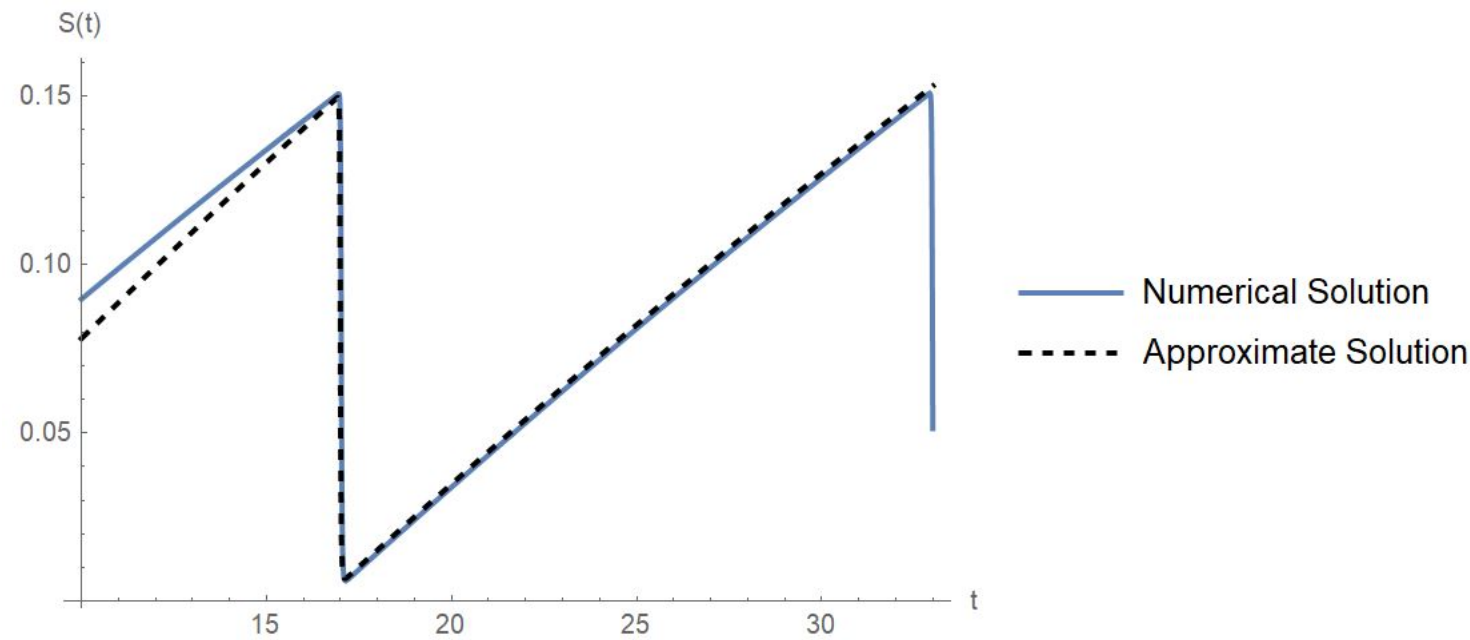

Figure 22: Numerical $S(t)$ and $\tilde{S}_{U}(t)$ as in equation (101) plotted over more than one period. Note that while the approximation is inaccurate for $t<17$, it remains highly accurate up to the point of the next outbreak. 


\section{Evolution of Long-Period Population Orbits}

Now that a closed-form approximate solution of the S-SIR model has been found, a study of the evolution of the system as it passes through outbreak and inter-outbreak regions may be performed. Of particular interest is the determination of conditions on the existence of periodic solutions for given parameter values. In the pursuit of this goal, the next step for this investigation is the derivation of a Poincaré map for the system, then determining the existence of fixed points of said mapping.

To enable the development of this mapping, an algorithm for determining the "key" points in the system trajectory will first be found. Using the notation described in Section 5.1, these key points are found at

$$
\begin{gathered}
\tilde{E}_{0}^{n}=\tilde{E}^{n}\left(t_{0}^{n}\right), \quad \text { where } t_{0}^{n} \text { is the beginning of the } n^{t h} \text { outbreak region } \\
\tilde{E}_{1}^{n}=\tilde{E}^{n}\left(t_{1}^{n}\right), \quad \text { where } t_{1}^{n} \text { is the end of the } n^{t h} \text { outbreak region } \\
\tilde{E}_{0}^{n+1}=\tilde{E}^{n+1}\left(t_{0}\right) \quad \text { where } t_{0}^{n+1} \text { is the beginning of the }(n+1)^{t h} \text { outbreak region. }
\end{gathered}
$$

The goal then is to develop a Poincaré mapping $P$ that maps the state of the system from outbreak to outbreak. In other words, we want to find $P$ such that $P\left(\tilde{E}_{0}^{n}\right)=\tilde{E}_{0}^{n+1}$.

\subsection{Determining Post-Outbreak Populations}

Starting from a pre-outbreak point $\left(S_{0}, I_{0}\right)$, we may use the approximate solution in Region I to to determine the populations after the outbreak. In order to proceed, caution must be taken: the dynamics of the system in the outbreak region are solely determined by $S_{0}$ and $I_{0}$, but we cannot assume that $S_{0} \approx 1$. In fact, making such an assumption will cause the generated results to vastly over-estimate the speed of the outbreak.

\subsubsection{Modifying the Outbreak Approximation}

To proceed, we must briefly return to considering the equations being discussed in the context of their application. The approximate solution for the outbreak region derived in Section 6 is based upon the assumption that $\mathrm{S}(0)$ is close to unity. When considering the evolution of a periodic mode beyond the initial outbreak, this is highly unlikely to hold. It is possible, however, to develop an adjusted form of the solution.

Definition 3. Let $N_{i}=S_{0}^{i}+I_{0}^{i}$ be known as the active population for the $i^{\text {th }}$ outbreak. 
We can consider $N_{i}$ to be the "active" population, as the dynamics of the system in the $S-I$ plane are entirely independent of $R$. In effect, we want to determine a way to transform from a solution to the SIR equations with $S(0)=e^{-\iota}, I(0) \approx 1-e^{-\iota}$ to one where $S(0)=N_{n} e^{-\iota}$ and $I(0)=N_{n}\left(1-e^{-\iota}-\frac{\iota}{r 0}\right)$. We can do this by introducing $S^{*}=\frac{S}{N_{n}}$ and $I^{*}=\frac{I}{N_{n}}$. This transformation yields

$$
\begin{aligned}
& \left(S^{*}\right)^{\prime}(t)=-N_{n} \beta_{0}\left(1+\delta_{0}\right)\left(S^{*}\right)\left(I^{*}\right) \\
& \left(I^{*}\right)^{\prime}(t)=N_{n} \beta_{0}\left(1+\delta_{0}\right)\left(S^{*}\right)\left(I^{*}\right)-\gamma I^{*} \\
& \left(S^{*}\right)(0)=e^{-\iota} \\
& \left(I^{*}\right)(0)=1-e^{-\iota}-\frac{\iota}{R_{0}} .
\end{aligned}
$$

Thus, the adjusted solution will be

$$
\begin{gathered}
\tilde{S}_{I}^{n}(t)=N_{n} \tilde{S}^{*}{ }_{I}(t), \quad \text { where } R_{0} \mapsto N_{n} R_{0} \\
\tilde{I}_{I}^{n}(t)=N_{n} \tilde{I}^{*}{ }_{I}(t), \quad \text { where } R_{0} \mapsto N_{n} R_{0}
\end{gathered}
$$

\subsubsection{Calculating the Initial Infected Population}

We may consider beginning at ${ }^{3} t=t_{0}$, the time immediately pre-outbreak. Contextually, we may expect then that $S^{\prime}\left(t_{0}\right)=0$ and $I^{\prime}\left(t_{0}\right)>0$. As this is the transition point between the validity of the assumptions made in Regions I and II, we must use the full S-SIR system to determine $S^{\prime}$. Let $^{4} \delta_{0}=\delta \cos \left(2 \pi t_{0}\right)$. We then expect

$$
\mu-\mu S_{0}-\beta_{0}\left(1+\delta_{0}\right) S_{0} I_{0}=0 .
$$

This allows a way of reducing the two degrees of freedom in specifying $E_{0}=\left(S_{0}, I_{0}\right)$ down to one. Given $S_{0}$, we may rearrange equation (103) for $I_{0}$, resulting in

$$
\left(I_{0}\right)_{S_{0}}=\frac{\mu\left(1-S_{0}\right)}{\beta_{0}\left(1+\delta_{0}\right) S_{0}} .
$$

\subsubsection{Determining the Outbreak Endpoint: Method A}

In order to continue forwards, a definitive point to match the Region I and Region II solution must be found. Due to the nature of the approximations made, there is no region where both approximate solutions are valid; instead, merely a region where both are not valid. As such, a smooth connection of the approximations is not possible. Instead, we may rely on the logic of

\footnotetext{
${ }^{3}$ Unless confusion is risked, the superscript $n$ will be suppressed for the subsequent discussion.

${ }^{4}$ Note that, as previously discussed, we expect $t_{0} \approx n$, where $n \in \mathbb{Z}$ for periodic modes.
} 
the application to guide the determination of the endpoint. Though we are using the simplified form introduced in Section 5.3 to model the trajectory during the outbreak, and that form lacks an equilibrium solution with non-zero infected population, we may return to the use of the full S-SIR model for the purpose of finding the extrema of $S^{\prime}$. When $S$ is at its minimum, we have transitioned from outbreak into inter-outbreak (or recovery, alternatively). As such, we must find $t_{1}$ such that

$$
\mu-\mu \tilde{S}_{I}\left(t_{1}\right)-\beta_{0}\left(1+\delta \cos \left(2 \pi t_{1}\right)\right) \tilde{I}_{I}\left(t_{1}\right) \tilde{S}_{I}\left(t_{1}\right)=0 .
$$

Clearly, the present form is an ill omen for the algebraic solvability of the equation. Upon substitution of equation (94), the result is the following labyrinthine equation.

$$
\begin{aligned}
& \mu-\exp \left(\frac{-G_{\infty} W_{0}\left(b_{1} e^{b_{1}-a_{1}\left(t_{1}\right)}\right)}{b_{1}}\right) \times \\
& \left(\mu-\beta_{0}\left(1+\delta \cos \left(2 \pi t_{1}\right)\right)\left\{1-\exp \left(\frac{-G_{\infty} W_{0}\left(b_{1} e^{b_{1}-a_{1}\left(t_{1}\right)}\right)}{b_{1}}\right)-\frac{-G_{\infty} W_{0}\left(b_{1} e^{b_{1}-a_{1}\left(t_{1}\right)}\right)}{b_{1}}\right\}\right)=0 .
\end{aligned}
$$

Unsurprisingly, such an expression has no chance of being solved algebraically. A root-finding algorithm must then be applied to determine $t_{1}$.

Upon application of a suitable algorithm, the results will yield the values of $S_{1}^{n}$ and $I_{1}^{n}$ necessary for proceeding.

\subsubsection{Determining the Outbreak Endpoint: Method B}

The final method that will be discussed is the most rooted in heuristics while also being the one that best lends itself to direct, closed-form expression without excessively reducing accuracy. In the work by Kroger, it is shown that the product $S(t) I(t)$ takes a form that may be approximated as a Gaussian curve. Associated with this is a well-defined Gaussian Width in the terminology of said paper, more commonly known as the standard deviation.

$$
\omega=\frac{4 G_{\infty}}{\sqrt{\pi}(\gamma+\mu)\left\{\left(G_{0}-R_{0}\right)^{2}-1\right\}} .
$$

It is a well-known result that $99.7 \%$ of the area beneath a Gaussian/Normal distribution will be found within six standard deviations [20]; thus we may use standard deviation to create an approximation of the total width of a given outbreak,

$$
t_{1}-t_{0} \approx W=\frac{24 G_{\infty}}{\sqrt{\pi}(\gamma+\mu)\left\{\left(G_{0}-R_{0}\right)^{2}-1\right\}},
$$


where $G_{\infty}$ must be calculated using the pre-outbreak population-corrected form of $\beta_{0}$ in order for accurate results to be achieved.

Unless stated otherwise, the following discussion will be carried out using this outbreak width as an approximation for the duration of the outbreak.

\subsection{Finding the Inter-Outbreak Region Using the Outbreak Width}

Having determined outbreak width in equation (108), we may then approximate the inter-outbreak period using $t_{C}$ from equation (29); specifically, we find

$$
\left.T \approx \frac{1}{\mu} W_{0}\left(\beta_{0} \phi^{-1}\left(1-\tilde{S}_{U}^{n}\left(t_{0}^{n}+W\right)\right)\right) e^{\frac{\mu\left(1+\ln I_{I}^{n}\left(t_{0}^{n}+W\right)\right)}{\phi}}\right)-\frac{\left(c+\ln I_{I}^{n}\left(t_{0}^{n}+W\right)\right)}{\phi} .
$$

\subsection{Poincaré Mapping and Variational Form}

After using one of the methods discussed previously to find $S_{1}^{n}$ and $I_{1}^{n}$, we are now equipped with everything needed to determine the Poincaré mapping for the dynamical system.

Given an inter-outbreak period $T$ and an initial susceptible population $S_{0}^{n}$, we can calculate $t_{1}$ by determining the root of the expression (106) or using equation (109). We may then use $\tilde{S}_{U}(t)$ given by equation (101) and $\left(I_{0}\right)_{S_{0}}$ determined using equation (104) to determine $S_{0}^{n+1}$ and $I_{0}^{n+1}$. Thus, let the function $P$ be defined as

$$
P_{T}\left(S_{0}^{n}\right)=\left(\begin{array}{c}
\exp \left(-G\left\{T-\left(t_{0}+W\right)\right\}\right)+1-e^{-\mu\left(T-\left(t_{0}+W\right)\right.} \\
\frac{\left(S_{0}+I_{0}\right) \mu\left(\left(S_{1}^{n}-1\right) e^{-\mu T}\right)}{\beta_{0}\left(1+\delta_{0}\right) S_{1}^{n}}
\end{array}\right)=\left(\begin{array}{c}
S_{0}^{n+1} \\
I_{0}^{n+1}
\end{array}\right) .
$$

Having determined the Poincaré Mapping for the system, it is now possible to investigate the existence of periodic modes in an algebraic or iterative way. Particularly, we may now see that if $S_{0}^{n}$ is such that there is a solution to the equation

$$
S_{0}^{n}+e^{-\mu\left(T-\left(t_{0}+W\right)\right.}-\exp \left(-G\left\{T-\left(t_{0}+W\right)\right\}\right)-1=0,
$$

then there exists a periodic solution with period $T$.

We may note that this provides an explanation for the existence of multiple possible periodic modes for a single set of parameters. We can see that the existence of a periodic solution for a given set of parameters is primarily dependent on the pre-outbreak population. The introduction of randomized noise causes switching of modes (as shown in Greenman [9]), which can then be understood as being due to the random perturbation in $S_{0}$ being sufficient to place it at another 
value for which a periodic solution exists.

\section{Results}

With the findings of the preceding work, it is now possible to rigorously investigate the qualitative effect of varying system parameters. In particular, we wish to investigate the change in $I_{\max }$, which could be considered as the measurement of the severity of an outbreak as the system parameters are varied.

To investigate this, $S_{0}-P\left(S_{0}\right)$ was repeatedly calculated as $S_{0}$ was varied by a small amount. When two consecutive values were opposite in sign, the middle-point between the two was taken as an approximation of the fixed point of the Poincaré mapping. Once $S_{0}$ had been iterated over the desired range, $\delta$ was then changed by a small amount, and the process was repeated. Two plots of $S_{0}-P\left(S_{0}\right)$ are shown in Figure 23 .

From Figures 23 and 24, we may observe that there is a narrow region in the low- $\delta$ paradigm where a behaviour reminiscent of hysteresis can be observed. Noting that $I_{\max }$ varies directly with $S_{0}$, we may observe that if we begin with $S_{0}$ such that the system is in a period-one mode and then vary $\delta$, there is a specific point ( $\delta \approx 0.09$ in Figure $24 \mathrm{a}$ and $\delta \approx 0.1$ in Figure 24a) where the period-one mode would vanish and the system would fall into a higher-period mode corresponding to a greater value of $S_{0}$.

Additionally, the necessary framework has now been established to allow a cataloguing of all possible inter-outbreak periods for a given set of parameters. The majority of the possible interoutbreak periods found do not correspond to a fixed point of the Poincaré map, and so are not truly "stable". It may be noted that these possibilities could correspond to what are known as "exotic modes", or trajectories that must cycle through multiple outbreak periods before returning to their initial values.

Of particular interest is the existence of a (narrow) region in which there are multiple stable periodic modes. It is easily seen that based on the qualitative shape, it appears that there may be up to three stable periodic modes available, that two of the states will be close together and relatively low in period, while another relatively high-period mode will be found as well.

As $\delta$ is varied, we may see that the location of the highest-period mode varies smoothly (shown in Figure 28). There is a more complicated change in the qualitative shape of the curve in the lower $S_{0}$ values, which may lead to the introduction of either one or two new modes. If two lower-value 


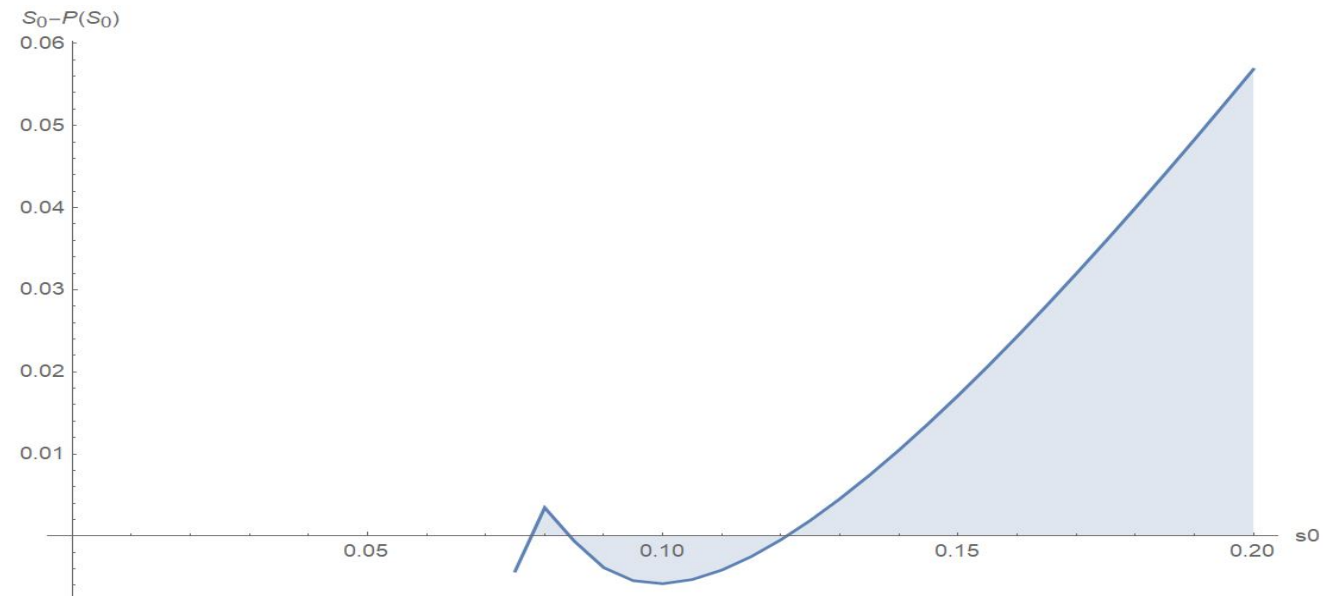

(a) $\delta=0.14$

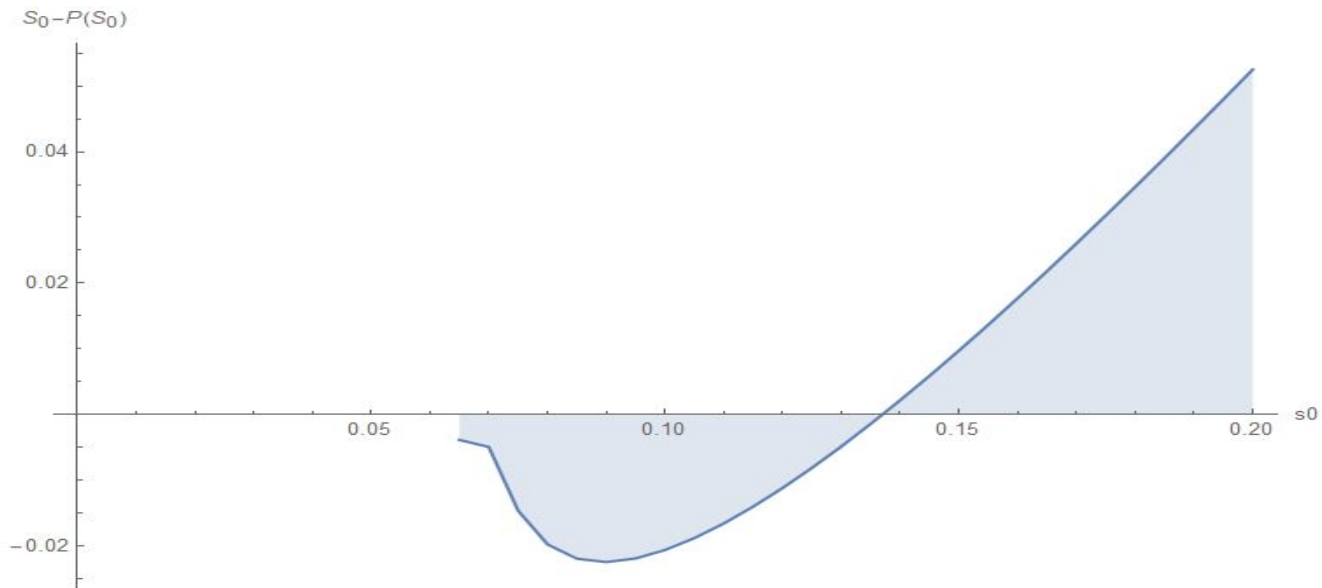

(b) $\delta=0.285$

Figure 23: $S_{0}-P\left(S_{0}\right)$ as $S_{0}$ is varied. The horizontal intercepts of the graph represent the existence of a stable periodic mode. Note the existence of three possible modes in (a). As $\delta$ is increased, the graph is distorted and shifted downward until only one root remains in (b). $\left(\beta_{0}, \gamma, \mu\right)=(500,40,0.01)$ 


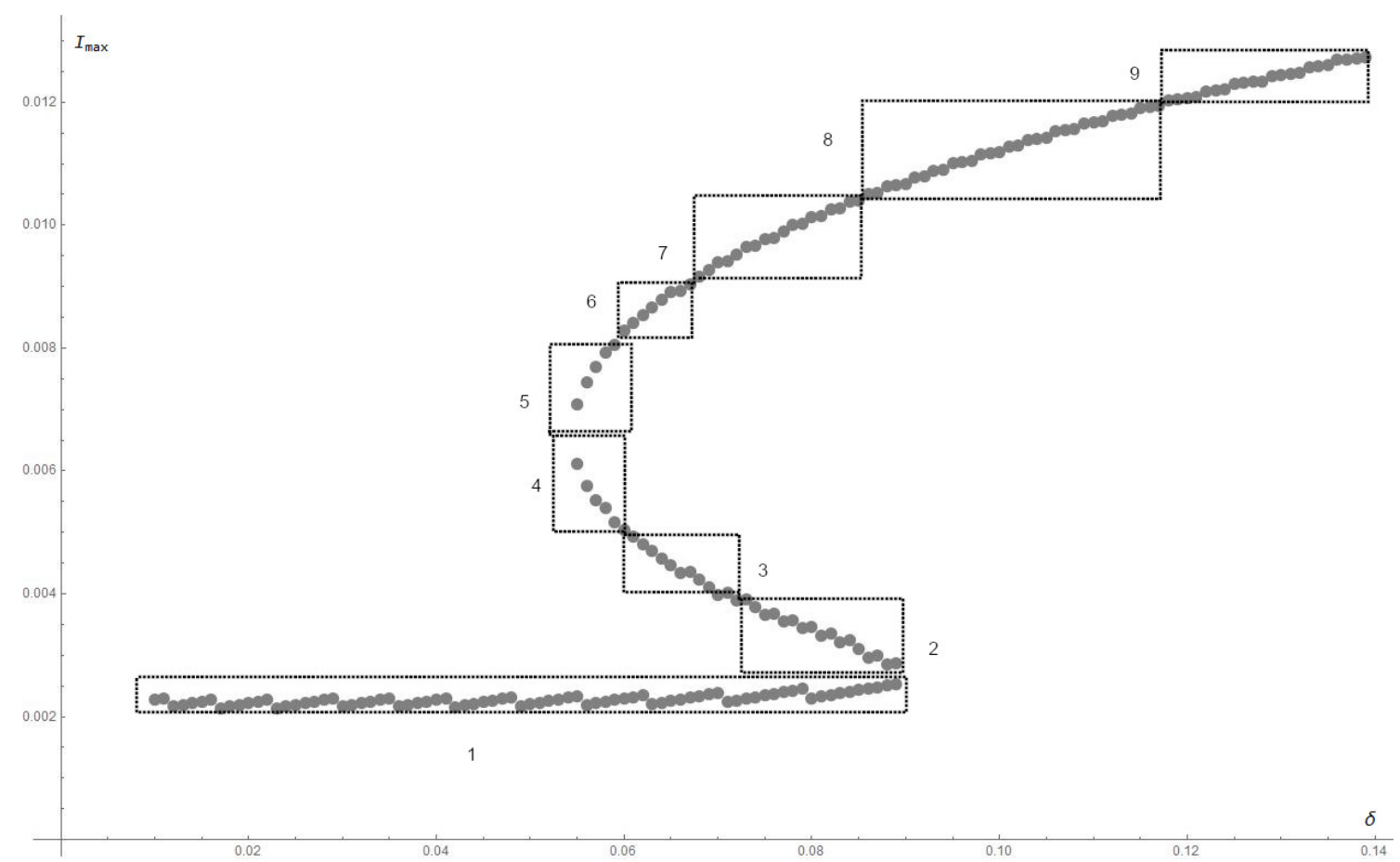

(a) $\left(\beta_{0}, \gamma, \mu\right)=(500,40,0.01)$. Note that this explains the phenomenon observed in 4 , particularly shown in figure 5

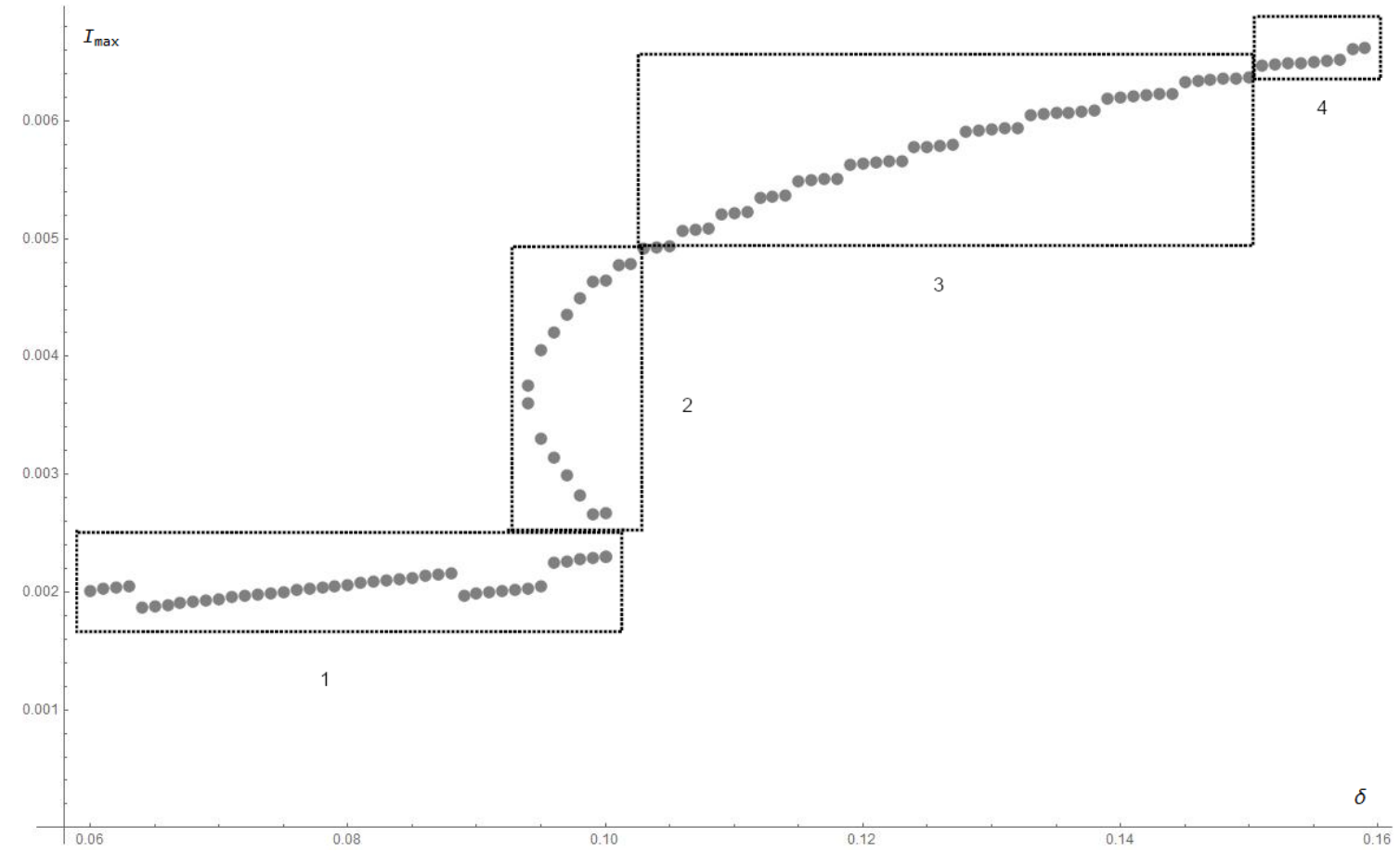

(b) $\left(\beta_{0}, \gamma, \mu\right)=(1300,40,0.01)$

Figure 24: $I_{\max }$ as $\delta$ is varied. The data points within each boxed region correspond to a grouping of modes sharing the same period (rounded to the nearest integer), as indicated by the labels outside each box. 


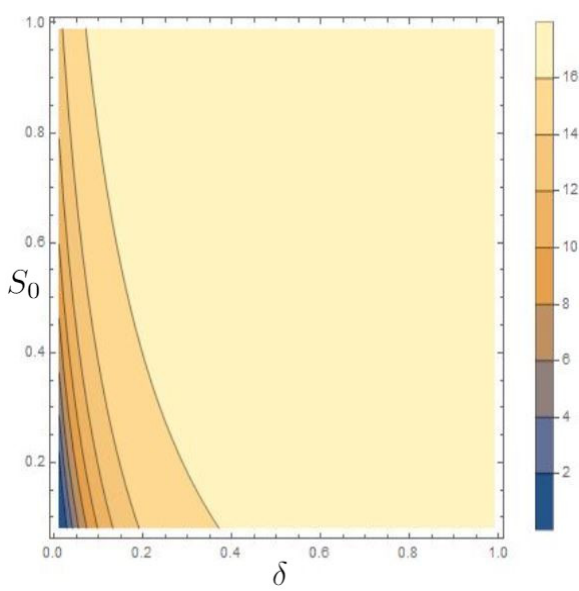

(a) $\gamma=40$

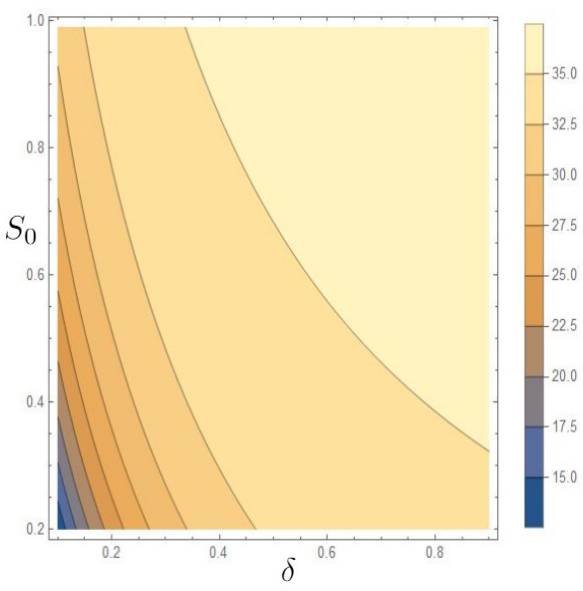

(b) $\gamma=80$

Figure 25: Contour plot of all possible inter-outbreak periods (without requiring they be integers) as $S_{0}$ and $\delta$ are varied, $\left(\beta_{0}, \mu\right)=(500,0.01)$

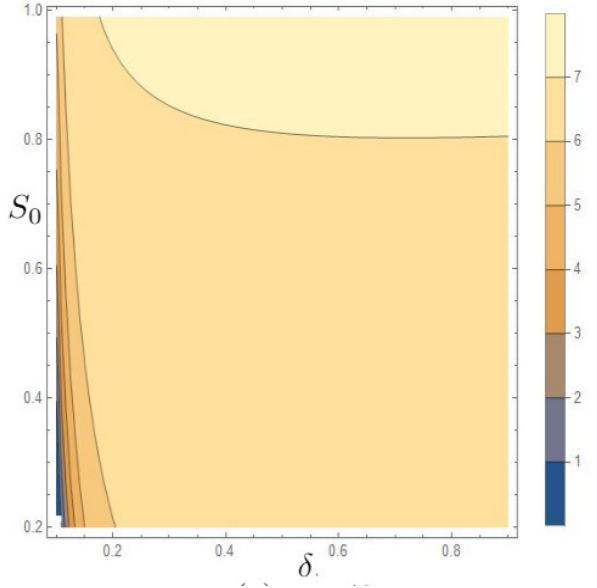

(a) $\gamma=40$

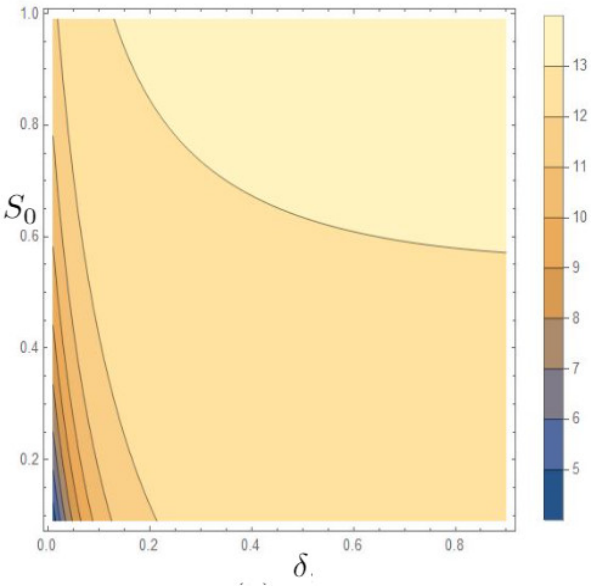

(b) $\gamma=80$

Figure 26: As in Figure 25, $\left(\beta_{0}, \mu\right)=(1300,0.01)$

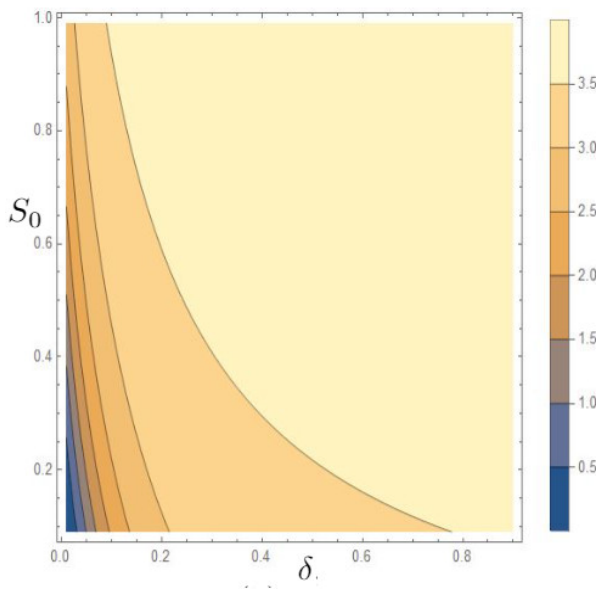

(a) $\gamma=40$

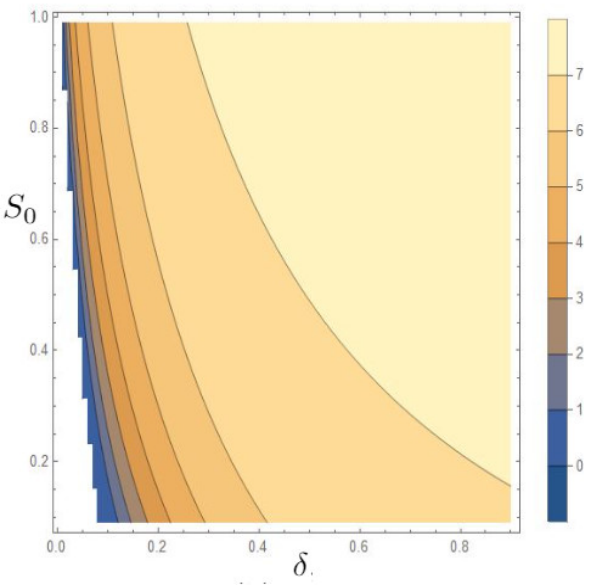

(b) $\gamma=80$

Figure 27: As in Figure 25, $\left(\beta_{0}, \mu\right)=(500,0.05)$ 


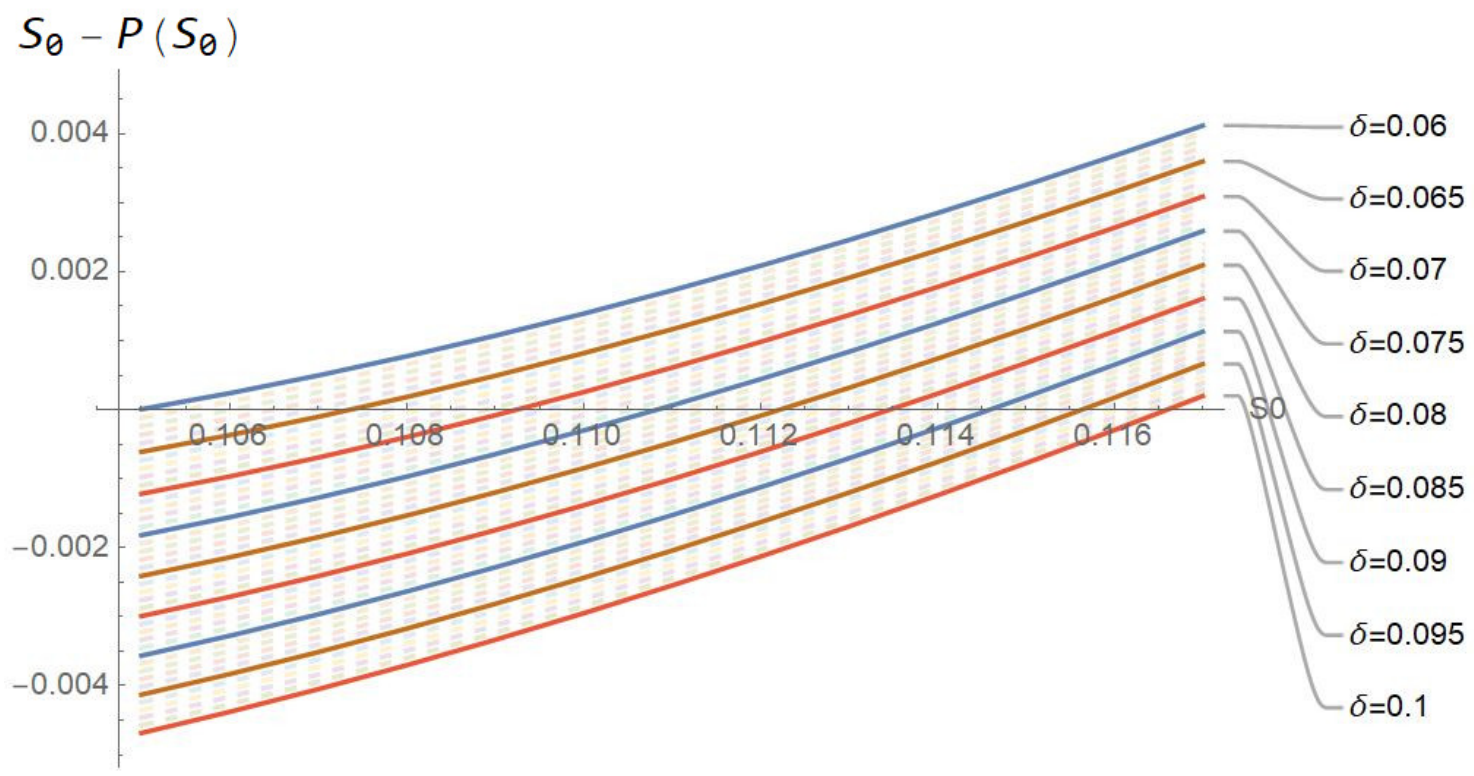

Figure 28: Plotting $S_{0}-P\left(S_{0}\right)$ as $S_{0}$ is varied for various values of $\delta$. Note that $\delta$ is incremented by 0.001 from curve to curve, with intervals of 0.005 indicated by the opaque lines.

roots are found, they will converge as $\delta$ is increased until they coincide, then disappear.

Figures 25, 26, and 27 show the entire spectrum of possible inter-outbreak periods for a given set of parameters, without the requirement of periodicity. We may see that for small values of $S_{0}$ and $\delta$, relatively low-period modes are predicted. This may be unreliable, as Period-one and Period-two modes do not exhibit the dramatically different scales of inter-outbreak populations between $S$ and $I$; thus the dominant balance arguments made for the construction of the inter-outbreak solution will not necessarily be valid.

Comparing the contour plots shown, a few key patterns emerge as the system parameters are varied. We may see that doubling $\gamma$ uniformly appears to nearly double the maximum interoutbreak period, while also distorting the placement of each period in the $\left(\delta, S_{0}\right)$ plane, though the distortion is not uniform. This result is consistent with the application context: as $\gamma$ is the rate of recovery/removal, a greater $\gamma$ suggests that there will be fewer infected individuals following an outbreak. Due to this reduced post-outbreak population, it takes longer for the infected population to return from near-extinction.

As $\beta_{0}$ is varied between Figures 25 and 26, we may observe that the highest inter-outbreak period available is reduced as $\beta$ is increased. Contextually, this reflects the intuitive notion that as the rate of infection is increased, it takes less time for the number of infected individuals to grow back to a critical population following near-extinction after an outbreak.

As $\mu$ is increased by $500 \%$ from Figure 25 to Figure 27, we may see that the entire spectrum of 
periods are decreased by approximately $500 \%$. Contextually, this suggests that a uniform increase in birth-rate/death-rate across the total population will reduce the inter-outbreak time, as the population of potential susceptibles will grow beyond the epidemic threshold more rapidly.

Guided by Figures 25-27, we may conclude that the majority of the spectrum of possible modes is dominated by the larger end and that there are a finite number of valid modes with a well-defined upper bound. Note that there has been no external requirement placed that the predicted period is an integer in the data displayed, as such the contour lines have been generated from a continuum of values. Experiment suggests that only integer values of periodicity are possible, but this is not guaranteed.

\section{Discussion}

\subsection{Comparison to Literature}

The results shown in Figures 23 and 24 coincide with observed results published by Greenman, Kano, and Boots in their numerical investigation of the S-SIR model [9]. Particularly, the available modes found and their corresponding values $\delta$ agree with their results, though their research indicated a more layered bifurcation structure, and their numerical results suggested that certain modes may exist in a wider region than shown here.

It is possible that multiple modes may be available past where they are indicated by Figures 23 and 24 , but that they cease to be truly stable modes. This suggests that the S-SIR model has a significantly different possible bifurcation hierarchy than that exhibited by the SEIR model, which has been shown to exhibit infinite subharmonic bifurcations [17].

Having shown that the model used is consistent with the results of previous research, the true test of the model is whether is able to predict the existence of periodic modes correctly ${ }^{5}$. Figures 29 and 30 show point-plots of available periods as $\delta$ is varied. Superimposed are plots of $S(t)$ from numerical simulations with corresponding parameter values and initial conditions.

Figure 29 shows that every predicted periodic mode is possible in the predicted parameter ranges. Note that the $T=3$ mode has noticeable variation from peak to peak, but the periodicity of the outbreaks is maintained. The fact that the procedure described in this section led to a prediction of a stable periodic mode for that value is consistent with the expected limitations of the approximate model: accuracy is expected to be reduced for lower-period modes.

\footnotetext{
${ }^{5}$ Specifically, existence of periodic modes in numerical simulation. As will be discussed in the Applications section, such modes are nearly impossible in reality.
} 


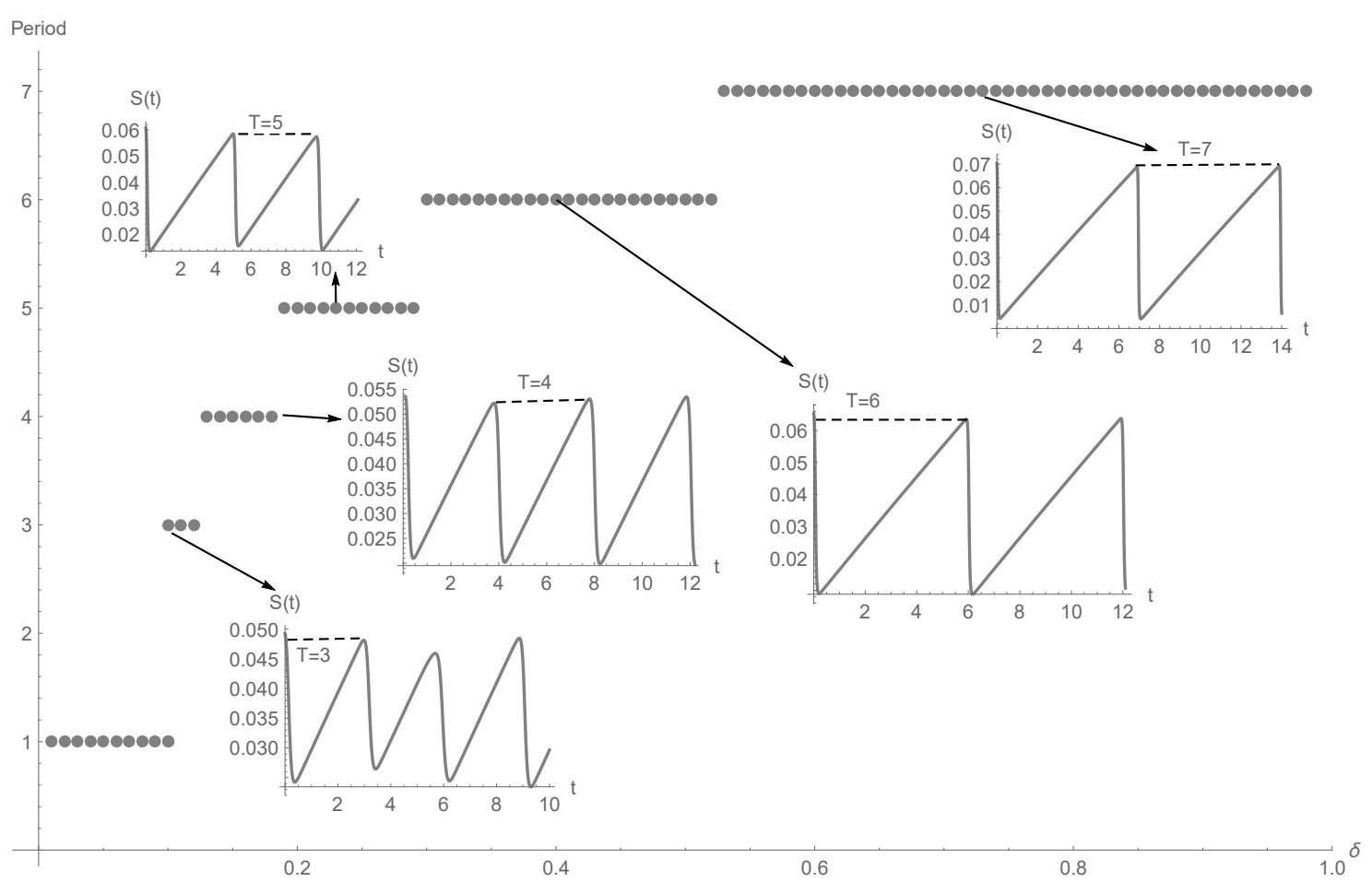

Figure 29: Plotting available periodic modes as $\delta$ varies. Shown next to each mode $T>1$ is a plot of $S(t)$, found numerically using the initial conditions and $\delta$ associated with the linked point. $\left(\beta_{0}, \gamma, \mu\right)=(1500,40,0.01)$

Figure 30 is the result of a similar procedure, but using the system parameter values measured for measles [9]. Notably, we may see that even the Period 1 mode was predicted correctly, but with relatively poor accuracy as indicated by the variation in peak values. The Period 2 mode shows increased variation, but the expected qualitative behaviour of having approximately two time units between each outbreak is shown to be correct. We may note that the Period 3 mode observed appears to show the least variation from peak-to-peak, suggesting we have entered the region where the model discussed performs best. Lastly, we may observe that the Period 4 mode found exhibits behaviour that has not been accounted for, having a small outbreak "early", before another more significant outbreak over the first four time units. Despite this unaccounted-for behaviour, we may note that the time between the major outbreaks is precisely the predicted four time units.

The close connection between increasing inter-outbreak period and increasing $I_{\max }$ as shown in Figures $24 \mathrm{a}$ and $24 \mathrm{~b}$ is unsurprising. It has been well documented ([8], [13], [16], [18]) that there exists a critical population density, or "epidemic threshold", dependent on the system parameters. In their pioneering work [13], Kermack and McKendrick noted

"The longer the epidemic is withheld, the greater will be the catastrophe, provided that the population continues to increase, and the threshold density remains unchanged. 


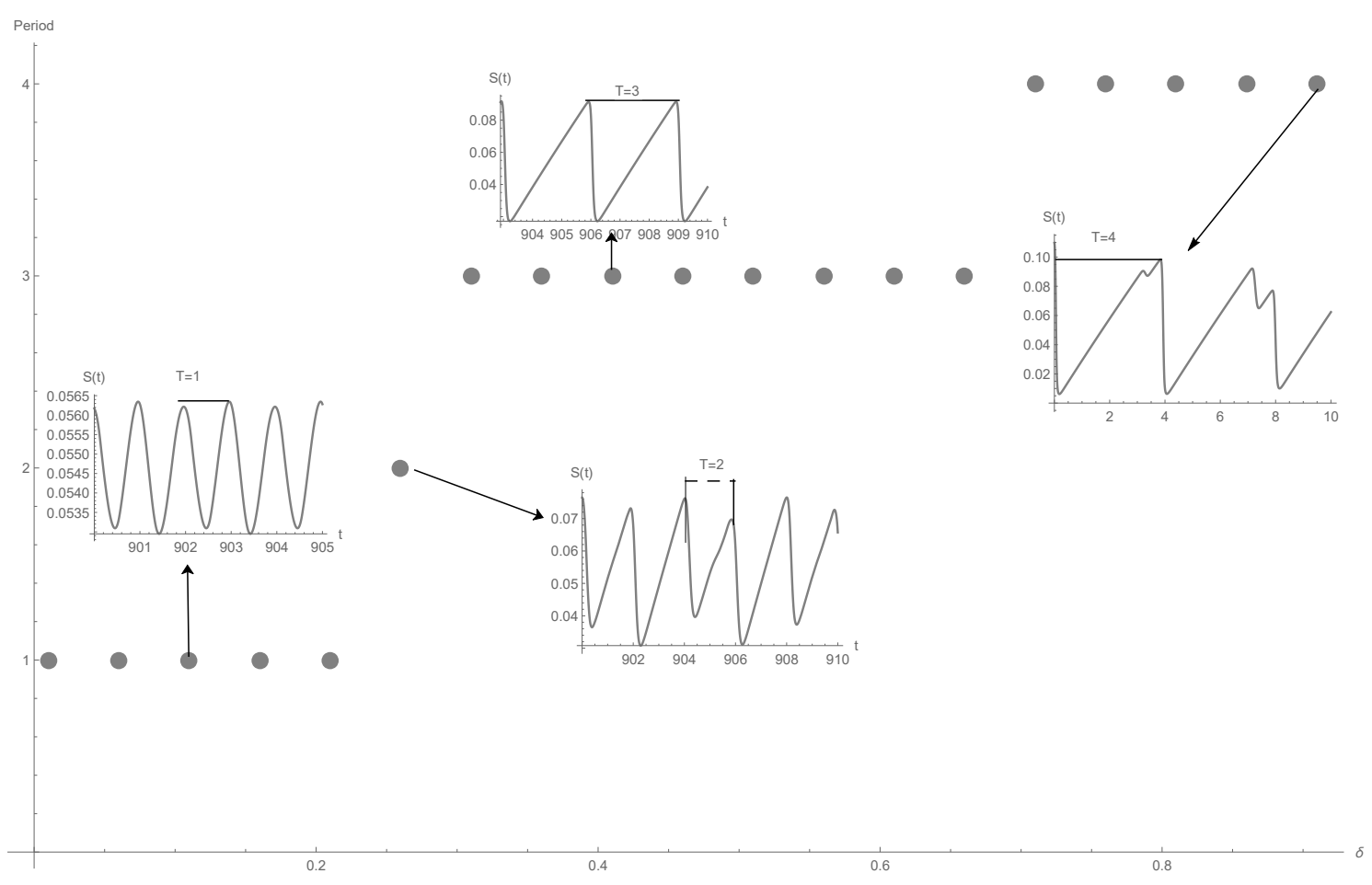

Figure 30: As in Figure 29, but with the system parameters of measles. $\left(\beta_{0}, \gamma, \mu\right)=(478,28,0.02)$

Such a prolonged delay may lead to almost complete extinction of the population."

The seasonal variation of the rate of infection may be considered as a way of delaying outbreak, so to speak. The longer the susceptible population has to recover following an outbreak, the greater the value of $S_{0}$. As was noted in Section 7.1.1, particularly equation (102), there is a direct scaling of $S$ and $I$ during the duration of an outbreak, proportional to their sum pre-outbreak.

We may also note that, from the necessary adjustments made in Section 7.1.1, and as explored in Appendix A, we may expect that the "effective" $R_{0}$ is scaled down proportionate to the preoutbreak active population. Shorter inter-outbreak periods result in smaller pre-outbreak populations, and thus a lowered effective $R_{0}$, which in turn will scale $u p$ the epidemic threshold, leading to outbreaks of reduced severity.

\subsection{Applications}

The purely mathematical results found serve to provide a possible explanation for the emergence of subharmonic modes for a seasonally varying rate of infection in numerical simulation, but these results additionally provide a clue as to the realism of said numerical simulations in the first place.

We may note that, as shown by Figure 31, the proportion of infected individuals will reach values on the order of $e^{-150}$ when in a state which leads to Period-sixteen mode. For this to be a non-zero 


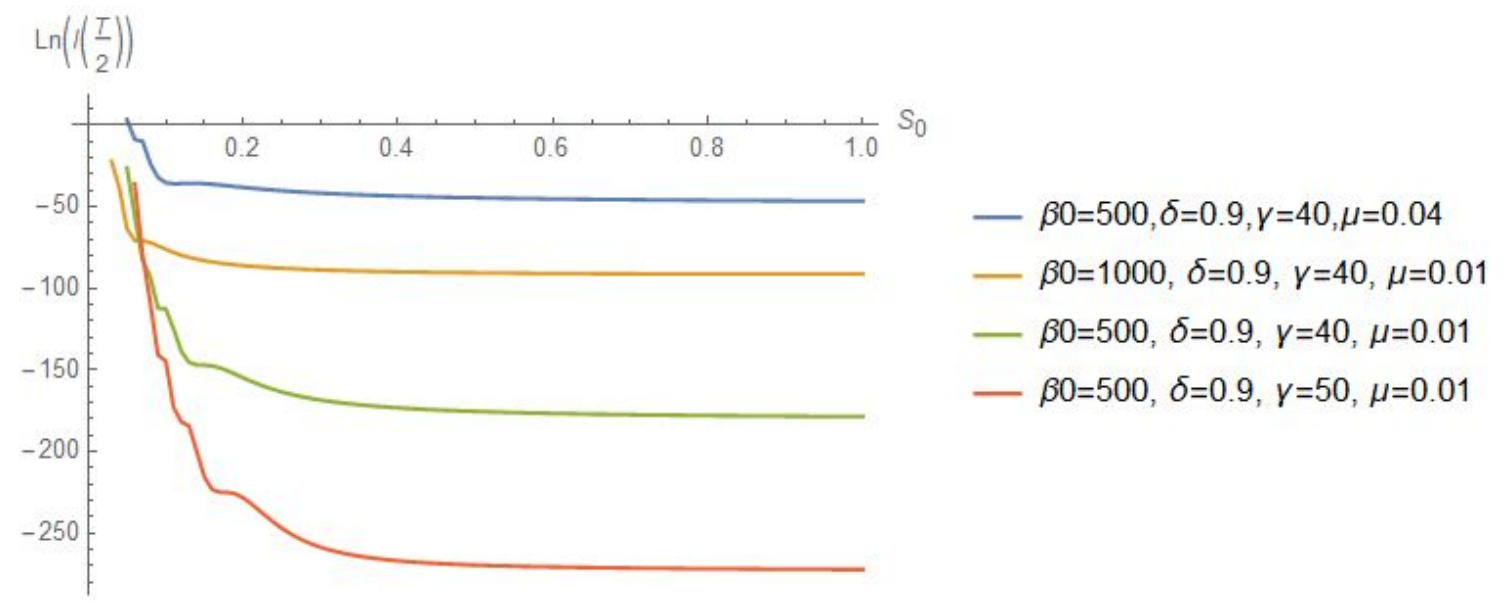

Figure 31: Using the value of $\tilde{I}_{I I}$ at the midpoint of the inter-outbreak period as a proxy for the minimum value, plotted as $S_{0}$ is varied

value, the total population must be impossibly large. We may then conclude that such population trajectories may exist only in the realm of mathematical models, and are not to be seen in any real-world populations of humans.

The enormous populations required may exclude the possibility of such periodic modes within human populations, but if one were to imagine an infection spread between individuals in a colony of microscopic organisms, such values are not necessarily entirely unrealistic. This is purely speculative, however, and such a circumstance would almost certainly lack the external periodic forcing in anything but a carefully constructed experiment, though the necessary conditions may be present for populations of microbes or viruses within a human body. 


\subsection{Future Research Opportunities}

While the results we have arrived at appear to be largely consistent with the numerical simulations that we intended to understand, it is still a fact that many approximations have been made in the attempt to construct a closed-form expression for the Poincaré mapping. Approximations of increased accuracy are desirable, though their existence is not necessarily guaranteed.

In Section 6.6, the approximation $\tilde{S}_{U}^{n}(t)$ was found, allowing an expression that had the correct asymptotic behaviour in both the outbreak and inter-outbreak regions. If an equivalent $\tilde{I}_{U}^{n}(t)$ were to be determined, then we may expect the accuracy of our results to be improved further. Exact results would then no longer require the numerical root-finding or Gaussian-width-based approximation needed to find the initial values for the inter-outbreak region, as was done for the results shown here.

A logical next step in the continuation of the present line of inquiry is the variation of the frequency of the seasonal forcing. In the research by Greenman et al. [9], they were able to construct resonantresponse diagrams as the forcing period was varied. The successful implementation of such variable forcing period would theoretically allow a resonance analysis of the system to be performed, similar to the work published by Amundsen, Cox, and Mortell on shallow fluids [1].

The results shown indicate that the severity of each outbreak is directly proportional to the interoutbreak period, but there may be worthwhile information to be gained by taking a resonancebased approach. As can be seen in Figure 3b, the V-SIR model without seasonal forcing exhibits a decaying oscillation into endemic equilibrium. Linearization about the endemic equilibrium suggests that there is a natural frequency of oscillation for the system near such a point [10]. Investigation into the relationship between this natural oscillation frequency and the available modes may prove insightful but is beyond the scope of the work at hand.

A possible direction that may bear meaningful results is the generalization of the system into models with multiple vector populations ([5], [11]), or to alternative models such as the SusceptibleExposed-Infected-Removed, Susceptible-Infected-Susceptible, or any of the other multitude of compartmental models for the spread of disease.

A final avenue that bears exploration is the much more difficult task of attempting a global analysis of the system, as opposed to the localized, iterative approach discussed here. Such an analysis may explain why the system falls into a particular mode. 


\section{Conclusion}

We have successfully answered the "guiding question" raised in Section 4. Particularly, we may conclude:

Under a seasonally varying rate of infection, periodic modes with long inter-outbreak periods will emerge due to the low-population dynamics exhibited by $I(t)$. The population of $S(t)$ may be sufficiently large, and $I(t)$ may be sufficiently small that $I(t)$ is affected by $S(t)$, but the reverse will not be true.

Under such conditions, it is possible for $\ln I(t)$ to undergo an arcing trajectory approximated by equation (26) such that the unscaled population will appear to be near-zero until an apparent sudden outbreak. The time from outbreak to outbreak may be approximated using equation (29).

By determining $t_{C}$, it is possible to construct a Poincaré mapping from pre-outbreak state $E_{0}^{n}$ to pre-outbreak state $E_{0}^{n+1}$. The fixed points of the Poincaré mapping indicate stable periodic modes.

Without imposing the requirement of periodicity, it is further possible to use $t_{C}$ to determine key traits such as the maximum severity of all possible periodic outbreaks and the necessary size of a population to sustain such periodic modes.

Using this method to investigate, we observe that, for any given set of parameters, there are a finite number of stable periodic modes, as well as a limited spectrum of inter-outbreak periods, whether or not the period corresponds to a fixed point of the Poincaré mapping.

We observe that doubling $\gamma$ leads to a near-doubling of the available inter-outbreak periods, that increasing $\beta_{0}$ leads to a decrease in the length of the inter-outbreak periods, and that increasing $\mu$ leads to a decrease in the length of the available interoutbreak periods.

Lastly, we have observed that for a given set of "intrinsic" system parameters $\beta_{0}, \gamma$, and $\mu$, low values of $S_{0}$ and $\delta$ correspond to low inter-outbreak periods, the spectrum of available periods is dominated by the upper range of availability, and there is a lower bound on possible $\delta$ and $S_{0}$ values. 


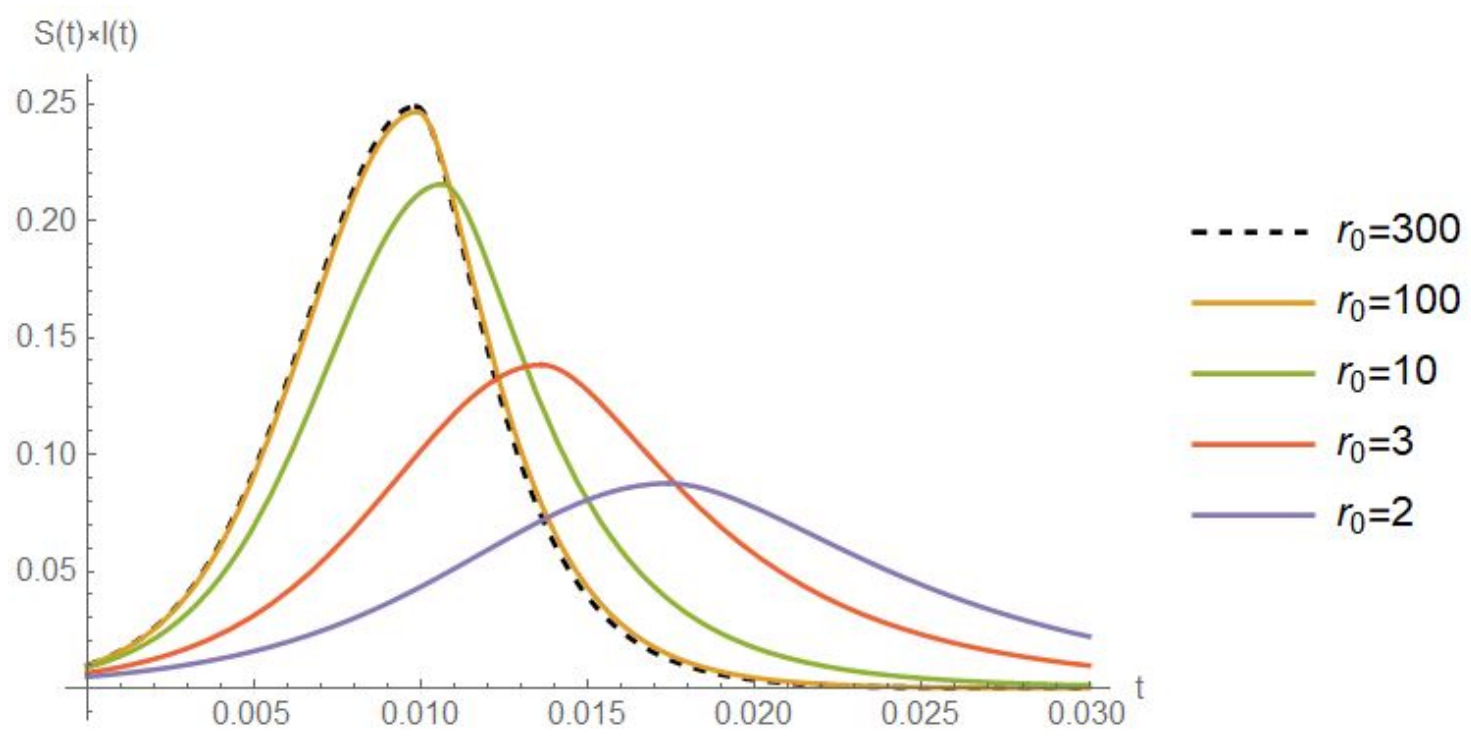

Figure 32: $j(t)=S(t) I(t)$ as $R_{0}$ is varied, with $S(0)=0.99$

\section{APPENDIX}

\section{A Qualitative Changes in Subsequent Outbreaks}

To begin, note that, as discussed in Section 7.1.1, we may use the "street-fighting" approximation for $E(t)$ to model the trajectory of the system within the outbreak region after applying an appropriate transformation to the parameters; specifically that $R_{0} \mapsto N_{n} R_{0}$. Displayed simply, we can observe that the total duration of an outbreak is proportional to the size of $R_{0}$ in Figure 32, approaching a limiting curve as $R_{0} \rightarrow \infty$. Note that $R_{0}=300$ is highly unrealistic, but is included to illustrate the asymptotic behaviour as $R_{0}$ grows large.

Since $N_{n}<1$, for all but the outbreak at the initial introduction of the disease, this transformation reflects the reduced speed at which the disease will spread through the susceptible population for subsequent outbreaks, as the susceptible population will be significantly lessened.

This transformation also reflects the fact that as $N_{n}$ increases, so will the effective $R_{0}$ value, indicating that the disease will only spread at its maximum possible rate when $N_{n} \approx 1$. It is only possible for $N_{n} \rightarrow 1$ if the inter-outbreak period $p$ grows large. The time for the susceptible population $\tilde{S}_{I I}(t)$, with initial value $S_{1}$ to reach a given value $y$ in the inter-outbreak period can be expressed as

$$
t=\frac{\ln \left(\frac{S_{1}-1}{y-1}\right)}{\mu},
$$

which clearly indicates that $t \rightarrow \infty$ as $y \rightarrow 1$. In summary, there exists a most-rapid outbreak for 
any given set of parameters, and the trajectory of the system in a periodic mode will only approach the curve of the most-rapid outbreak as the inter-outbreak period approaches infinity. The absolute lower bound for the width of any outbreak appears to be an outbreak width of approximately 0.02 . 


\section{References}

[1] D.E. Amundsen, E.A. Cox and Mortell. M.P. 'Asymptotic analysis of steady solutions of the KdVB equation with application to resonant sloshing'. In: Z.angew. Math. Phys. 58 (2007), pp. 1008-1034.

[2] The World Bank. URL: https://data.worldbank.org/indicator/SP.DYN.TFRT.IN.

[3] C. M. Bender and S. A. Orszag. Advanced Mathematical Methods for Scientists and Engineers: Asymptotic Methods and Perturbation Theory. New York: McGraw-Hill, 1978.

[4] CDC. COVID-19 Pandemic Planning Scenarios. 2021. URL: https://www.cdc.gov/coronavirus/ 2019-ncov/hcp/planning-scenarios.html.

[5] D.Z. Childs and M. Boots. 'The interaction of seasonal forcing and immunity and the resonance dynamics of malaria'. In: J.R. Soc. Interface 7 (2010), pp. 309-319.

[6] R.M. Corless, G. H. Gonnet and D. E. G. Hare. 'On the Lambert W Function'. In: Advances in Computational Mathematics 5 (1996), pp. 329-359.

[7] B. Denholm and C. Forbes. 'A model for the dynamics of Ross River Virus in the Australian environment'. In: Letters in Biomathematics 4 (2017), pp. 187-206.

[8] K. Dietz. 'The Incidence of Infectious Diseases Under Seasonal Fluctuations'. In: Mathematical Models in Medicine. Lecture Notes in Biomathematics 11 (1976), pp. 1-15.

[9] J. Greenman, M. Kamo and M. Boots. 'External forcing of ecological and epidemiological systems: a resonance approach'. In: Physica D (2004), pp. 136-151.

[10] I. Gumowski, C. Mira and R. Thibault. 'The incidence of infectious diseases under the influence of seasonal fluctuations - Periodic modes'. In: Proceeding of the First world conference on mathematics at the service of man 9 (1977), pp. 140-156.

[11] M. Kamo and Sasaki. A. 'The effect of cross-immunity and seasonal forcing in a multi-strain epidemic model'. In: Physica D 165 (2001), pp. 228-241.

[12] G. Katriel. 'Existence of Periodic Solutions For the Periodically Forced SIR Model'. In: $J$. Math. Sciences 201 (3 2014).

[13] W. Kermack and A. McKendrick. 'Contributions to the Mathematical Theory of Epidemics — I'. In: Bulletin of Mathematical Biology 53 (1 1927), pp. 33-35.

[14] M. Kroger and R. Schlickeiser. 'Analytical solution of the SIR-model for the temporal evolution of epidemics. Part A: time-independent reproduction factor'. In: J. Phys. A: Math. Theor. 53 (2020), pp. 245-283. 
[15] J. Liu. 'Threshold dynamics for a HFMD epidemic model with periodic transmission rate'. In: Nonlinear Dyn 64 (2011), pp. 89-95.

[16] A.J Lotka. 'Contribution to the Analysis of Malaria Epidemiology I: General Part.' In: Supplement to Amer. J. Hygiene 3 (1923), pp. 1-37.

[17] I. Schwartz and H.L. Smith. 'Infinite subharmonic bifurcation in an SEIR epidemic model'. In: J. Math. Biology 18 (1983), pp. 233-253.

[18] F.R. Sharpe and A.J. Lotka. 'Contribution to the Analysis of Malaria epidemiology IV: Incubation Lag'. In: Supplement to Amer. J. Hygiene 3 (1923), pp. 96-112.

[19] H.L. Smith. 'Subharmonic Bifurcation in an S-I-R Epidemic Model'. In: J. Math. Biology 17 (1983), pp. 163-177.

[20] E. Weisstein. Normal Distribution. URL: https://mathworld.wolfram.com/NormalDistribution. html. 
And so we come to our end, of sorts.

A play that contains multitudes:

All who died,

All who live,

All who yet shall,

Upon this stage with us

Forever.

CURTAIN

Impossible Landscapes, by Dennis Detwiller 